\title{
WestVirginiaUniversity
}

THE RESEARCH REPOSITORY @ WVU

Graduate Theses, Dissertations, and Problem Reports

2011

\section{Utilization of IGS Information for Improved Real-time GPS Positioning}

Francis James Barchesky

West Virginia University

Follow this and additional works at: https://researchrepository.wvu.edu/etd

\section{Recommended Citation}

Barchesky, Francis James, "Utilization of IGS Information for Improved Real-time GPS Positioning" (2011). Graduate Theses, Dissertations, and Problem Reports. 2294.

https://researchrepository.wvu.edu/etd/2294

This Thesis is protected by copyright and/or related rights. It has been brought to you by the The Research Repository @ WVU with permission from the rights-holder(s). You are free to use this Thesis in any way that is permitted by the copyright and related rights legislation that applies to your use. For other uses you must obtain permission from the rights-holder(s) directly, unless additional rights are indicated by a Creative Commons license in the record and/ or on the work itself. This Thesis has been accepted for inclusion in WVU Graduate Theses, Dissertations, and Problem Reports collection by an authorized administrator of The Research Repository @ WVU. For more information, please contact researchrepository@mail.wvu.edu. 


\title{
Utilization of IGS Information for Improved Real-time GPS Positioning
}

\author{
Francis James Barchesky
}

\author{
Thesis submitted to the \\ College of Engineering and Mineral Resources \\ at West Virginia University \\ in partial fulfillment of the requirements \\ for the degree of \\ Master of Science \\ in \\ Aerospace Engineering
}

Approved By:

Dr. Marcello Napolitano, Ph.D., Research Advisor

Dr. Yu Gu, Ph.D.

Dr. Powsiri Klinkhachorn, Ph.D.

Department of Mechanical and Aerospace Engineering

Morgantown, West Virginia

2011 
Utilization of IGS Information for Improved Real-time GPS Positioning

by Francis James Barchesky

The estimation of a precise user's position is a difficult and complex problem. In addition, the use of geodetic grade position instruments is often not possible for Small Unmanned Aerial Vehicle (SUAV) systems. However, the availability of the global navigation satellite system (GNSS) and International GNSS Service (IGS) predicted product data allows an attempt to increase the precision of a navigation algorithm, which is the aim in this thesis.

The utilization of this information within an algorithm work environment is a complex problem, requiring the development of multiple tools in order to use and access the IGS raw and product data. Therefore, the overall goal of this research project was the development of these tools using MATLAB ${ }^{\circledR}$. The IGS information provided by these tools allows access to a particular set of product and raw data files. The available predicted product data is used to increase the precision of the position estimate for a real-time application. Within this, the conversion from a long time interval to a fast update rate was determined. The use of this information requires these tools to also include important orbit determinations of the GPS satellites.

The use of only precise satellite position information from the developed MATLAB tools is evaluated by a comparison of a position estimation algorithm using recorded satellite position information and the developed satellite position information from the IGS predicted data. The results show an increase in performance of position estimation with the use of the created MATLAB tools. A discussion in how the use of the created tools could further be expanded to increase the accuracy and precision of a position estimation algorithm is presented. 


\section{Acknowledgements}

I would like to thank Dr. Marcello Napolitano, my research advisor, for your support, guidance and teachings throughout my education. I greatly appreciate all that you have done for me personally and my education.

Dr. Gu, throughout this process our discussions about this graduate project have been invaluable and greatly appreciated. I feel from these a significant improvement has been made within this project. I would like to also thank my other committee member, Dr. Powsiri Klinkhachorn for providing me with helpful feedback. Dr. Srikanth Gururajan and Dr. Brad Seanor your support and encouragement throughout my graduate studies and within the WVU flight testing activities have been greatly appreciated. Thank you so much Steve Raque for your support, advice, and help throughout my undergraduate and graduate studies.

To my fellow graduate student in the group: Jason Gross your advisement, discussions, and constant help were all appreciated, Matt Rhudy, your constant help and insight about problems

and writing was valued, Matteo Guerra our conversions and help with concepts of GPS was very useful. Zach Merceruio, and Marc Gramlich your construction and development of the PAC aircraft was an immeasurable task, I appreciate you and your work. Thank you Haiyang Chao for your development and help with avionics, it has been appreciated. Kerri Phillips, thank you for all your work in PID and advice. I want to thank Daniele Tancredi, your help and presence was cherished. Giovanni De Nunzio, thank you for your advice and help. Matteo Dariol your insight with respect to many issues was useful and appreciated. Thank you Amanda McGrail, your work and helpfulness was valued.

My girlfriend, Whitney Swesey, words cannot describe how appreciative I am for your love and support. To my family your love, consideration, enthusiasm and advisement has been inspirational to me. 


\section{Table of Contents}

$\begin{array}{lll}\text { CHAPTER } 1 & \text { : INTRODUCTION } & 1\end{array}$

$\begin{array}{llr}1.1 & \text { Problem Description } & 1\end{array}$

$\begin{array}{llr}1.2 & \text { Project Overview } & 2\end{array}$

1.3 Literature Review $\quad 3$

1.3.1 Simulating the Global Positioning System 3

1.3.2 Using International GNSS Service Product Data 4

$\begin{array}{llr}1.4 & \text { Organization } & 6\end{array}$

$\begin{array}{lll}\text { CHAPTER } 2 & \text { : BACKGROUND INFORMATION } & 7\end{array}$

$\begin{array}{lll}2.1 & \text { Global Positioning System } & 7\end{array}$

$\begin{array}{lll}2.1 .1 & \text { Global Positioning System History } & 7\end{array}$

2.1.2 GPS Signals 9

$\begin{array}{ll}\text { 2.1.3 User Position Determination } & 10\end{array}$

\begin{tabular}{ll}
2.1 .4 & Reference Frames \\
\hline
\end{tabular}

2.1.5 Satellite Position Determination $\quad 15$

$\begin{array}{ll}\text { 2.1.6 GPS Error Sources } & 19\end{array}$

$\begin{array}{lll}2.2 & \text { International GNSS Service (IGS) } & 23\end{array}$

$\begin{array}{llr}2.3 & \text { GPS/INS Navigation } & 26\end{array}$

$\begin{array}{llr}2.4 & \text { Extended Kalman Filter } & 27\end{array}$

$\begin{array}{lll}\text { CHAPTER } 3 & \text { : EXPERIMENTAL SET-UP } & 28\end{array}$

$\begin{array}{llr}\text { 3.1 WVU Propulsion Assisted Control Aircraft } & 28\end{array}$

3.2 Propulsion Assisted Control Aircr aft Avionics $\quad 30$

3.2.1 Gen-VOverview $\quad 31$

3.2.2 Custom Enclosure $\quad 32$

3.2.3 Peripheral Connection Board of Gen-V

3.2.4 Signal Distribution Board of Gen-V

$\begin{array}{lll}3.2 .5 & \text { Nose Avionics Board } & 39\end{array}$

3.2.6 External Signal Distribution Board 41

$\begin{array}{lll}3.3 & \text { WVU Flight Testing } & 43\end{array}$

CHAPTER 4 : DEVELOPMENT OF MATLAB TOOLS 45 
4.2 Initial Orbit Determination

4.3 GPS Orbit Interpolation 


\section{List of Figures}

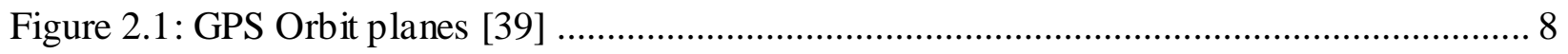

Figure 2.2: Range measurement timing relationships [43] ................................................. 10

Figure 2.3: Satellite geometry with parameters labeled Figure 6.2 [39] .................................. 13

Figure 2.4: 2-Dimension example of how GPS geometry affects position measurement [42] .... 20

Figure 2.5: Combined IGU Orbits compared to IGR [64] ................................................. 25

Figure 2.6: Recent week median difference between $24 \mathrm{hr}$ IGU and IGS products [64] ............ 25

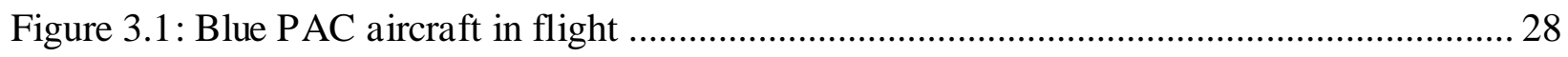

Figure 3.2: Current ducted fan in use on the PAC platform ................................................ 29

Figure 3.3: Locations of PAC aircraft avionics .............................................................. 30

Figure 3.4: Gen-V avionics stack in custom enclosure [68], [2] ........................................... 31

Figure 3.5 : Silk screen design placed on Gen-V enclosure.................................................. 32

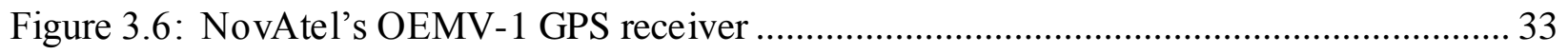

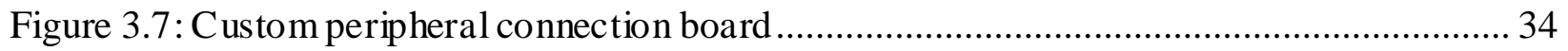

Figure 3.8: Cus tom peripheral connection block diagram .................................................. 35

Figure 3.9: Control mode activation block diagram ......................................................... 36

Figure 3.10: Bottom PCB fully assembled ....................................................................... 37

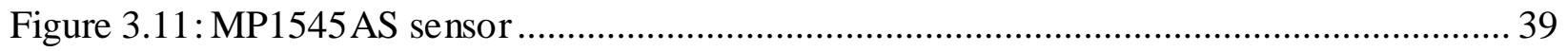

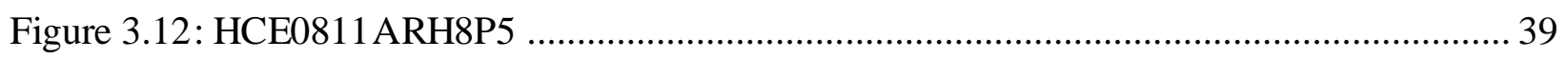

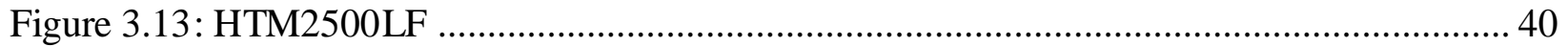

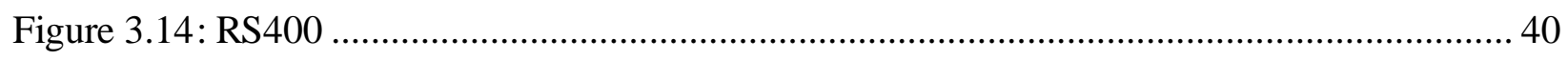

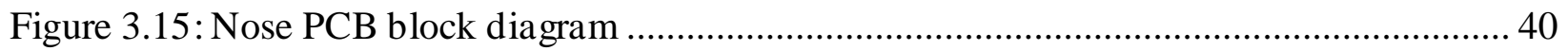

Figure 3.16: Fully assembled Blue/Green PAC aircraft nose PCB .......................................... 41

Figure 3.17: Spider board block diagram and fully assembled picture .................................... 42

Figure 3.18: Louis Bennet Field .................................................................................... 43

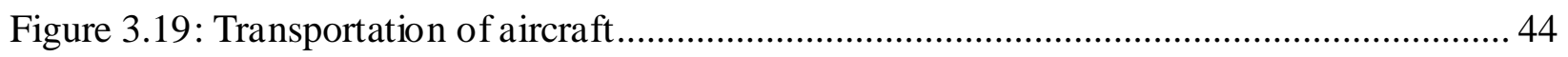

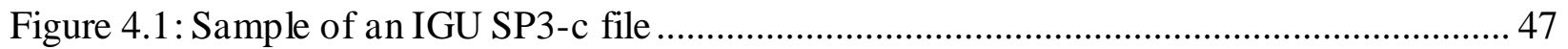

Figure 4.2: Sample of a RINEX file ................................................................................. 48

Figure 4.3: Position differences of broadcast data and IGU orbit .......................................... 49

Figure 4.4: Position Error of PRN-8 without a navigation message update .............................50

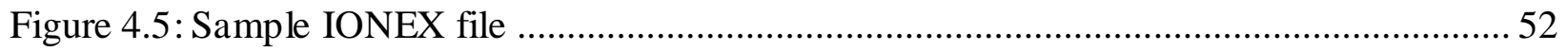

Figure 4.6: Total electron content change throughout a day .................................................. 53

Figure 4.7: Antenna viewing cone and Earth's shadow [84] .............................................. 54

Figure 4.8: GPS data play-back b lock diagram .................................................................... 55

Figure 4.9: PRN-1 satellite's separation angle throughout a dataset ........................................ 57

Figure 4.10: Method comparison of X-axis velocity estimates ...............................................5 57

Figure 4.11: ECEF position coord inates from precise ephemeral data ....................................59

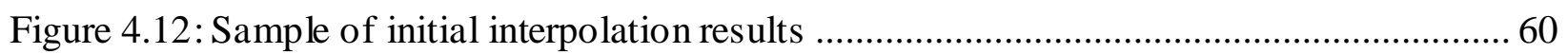

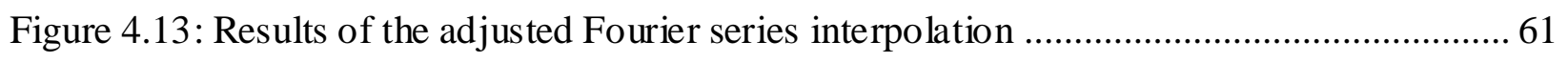




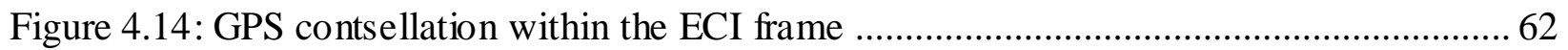

Figure 4.15: Satellite PRN-9 position throughout entire IGU dataset ..................................... 63

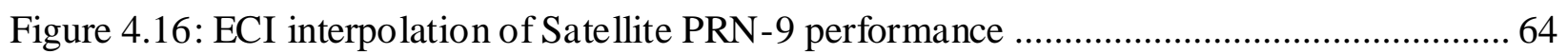

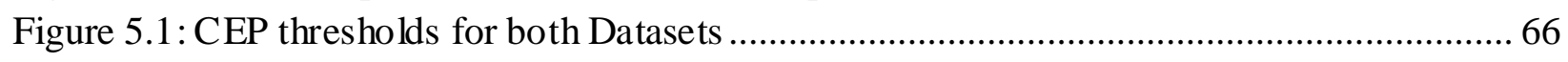

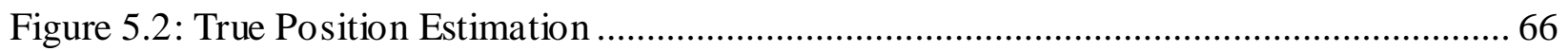

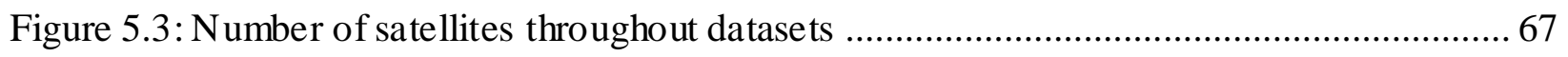

Figure 5.4: Sample of a refinement of SATXYZ dataset ..................................................... 69

Figure 5.5: Orthogonal views of Dataset-1 constellation geometry ...................................... 75

Figure 5.6: Radius of estimated position error for the Baseline estimation of Dataset-1 ........... 76

Figure 5.7: Radius of estimated position error for the CMTM estimation of Dataset-1 ............. 77

Figure 5.8: Radius of estimated position error for the Baseline estimation of Dataset-1 with removal of poorly positioned satellites .......................................................................... 78

Figure 5.9: Radius of estimated position error for the CMTM estimation of Dataset-1 with removal of poorly positioned satellites ......................................................................... 78

Figure 5.10: Radius of estimated position error for the Baseline estimation of Dataset-2 .......... 79

Figure 5.11: Radius of estimated position error for the CMTM estimation of Dataset-2 .......... 80

Figure 5.12: Orthogonal views of Dataset-2 constellation geometry ...................................... 80

Figure 5.13: Radius of estimated position error for the Baseline estimation of Dataset-2 with

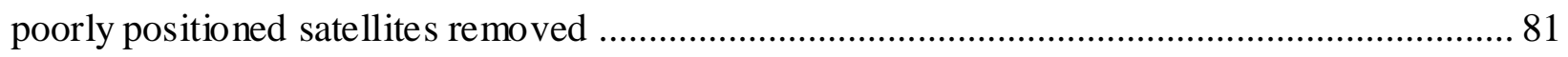

Figure 5.14: Radius of estimated position error for the CMTM estimation of Dataset-2 with poorly positioned satellites removed 


\section{List of Tables}

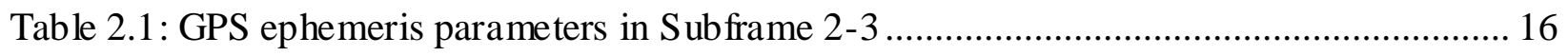

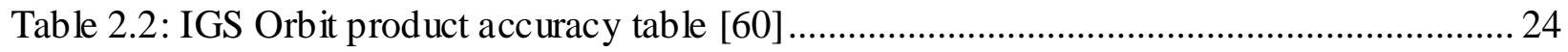

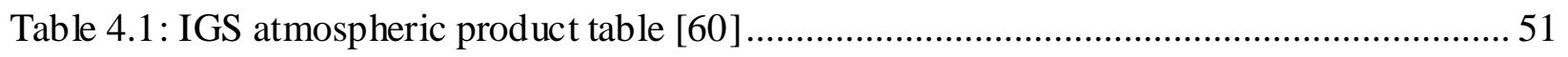

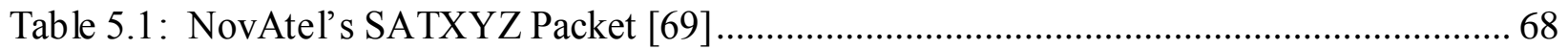

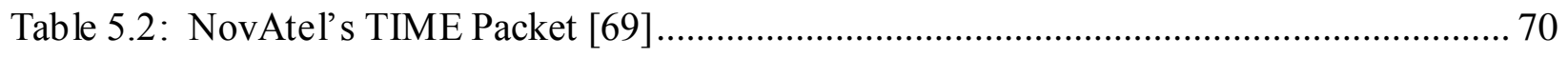




\section{List of Symbols}

\begin{tabular}{|c|c|c|}
\hline$a$ & $=$ & semimajor axis \\
\hline$a_{f 0}$ & $=$ & satellite clock bias \\
\hline$a_{f 1}$ & $=$ & satellite clock drift \\
\hline$a_{f 2}$ & $=$ & satellite clock drift rate \\
\hline$A$ & $=$ & prediction Jacobian matrix \\
\hline$b$ & $=$ & semiminor axis \\
\hline$c$ & $=$ & speed of light \\
\hline$C_{i c}$ & $=$ & amplitude of cosine correction to inclination angle \\
\hline$C_{i s}$ & $=$ & amplitude of sine correction to inclination angle \\
\hline$C_{r c}$ & $=$ & amplitude of cosine correction to orbital radius \\
\hline$C_{r s}$ & $=$ & amplitude of sine correction to orbital radius \\
\hline$C_{u c}$ & $=$ & amplitude of cosine correction to argument of latitude \\
\hline$C_{i c}$ & $=$ & amplitude of sine correction to argument of latitude \\
\hline$E$ & $=$ & eccentric anomaly \\
\hline$h$ & $=$ & altitude \\
\hline$H$ & $=$ & observation matrix \\
\hline$i$ & $=$ & inclination angle \\
\hline$I$ & $=$ & identity matrix \\
\hline$k$ & $=$ & discrete index \\
\hline$K$ & $=$ & Kalman gain matrix \\
\hline$M$ & $=$ & mean anomaly \\
\hline$P$ & $=$ & state covariance matrix \\
\hline$Q$ & $=$ & process noise covariance matrix \\
\hline$R$ & $=$ & state covariance matrix \\
\hline$t$ & $=$ & time of interest \\
\hline$t_{G P S}$ & $=$ & corrected satellite time of interest \\
\hline$t_{k}$ & $=$ & time from reference epoch \\
\hline$t_{o}$ & $=$ & epoch time \\
\hline$t_{o e}$ & $=$ & reference time of epoch \\
\hline$T_{s}$ & $=$ & system time at which the signal left the satellite \\
\hline$T_{u}$ & $=$ & system time at which the signal reached the user receiver \\
\hline$t_{u}$ & $=$ & offset of the receiver clock from system time \\
\hline$r$ & $=$ & geometric range \\
\hline$r_{\text {err }}$ & $=$ & radius of estimated position error \\
\hline$u$ & $=$ & input vector \\
\hline $\mathbf{x}$ & $=$ & state vector \\
\hline$\hat{\mathrm{x}}_{0}$ & $=$ & initialized state vector \\
\hline
\end{tabular}




$\begin{array}{lll}x_{j} & = & j \text { th-satellite } \mathrm{x} \text {-position } \\ x_{u} & = & \text { GPS receiver } \mathrm{x} \text {-position } \\ y_{j} & = & j \text { th-satellite } \mathrm{y} \text {-position } \\ y_{u} & = & \text { GPS receiver } \mathrm{y} \text {-position } \\ z & = & \text { measurement vector } \\ z_{j} & = & \text { Gth-satellite } \text {-position } \\ z_{u} & = & \text { offset of the satellite clock from system time } \\ \delta t & = & \text { change in time } \\ \Delta t & = & \text { relativistic correction term } \\ \Delta t_{r} & = & \text { corrected mean motion } \\ \Delta n & = & \text { latitude angle } \\ \lambda & = & \text { longitude of ascending node } \\ \varphi & = & \text { argument of perigee } \\ \Omega & = & \text { true anomaly } \\ \omega & = & \text { geocentric gravitational constant } \\ v & = & \text { atmospheric correction term }\end{array}$




\section{List of Acronyms}

\begin{tabular}{|c|c|c|}
\hline AGHS & $=$ & Advanced GPS Hybrid Simulator \\
\hline BPK & $=$ & Binary Phase-Shift Keying \\
\hline C/A & $=$ & Course/Acquisition \\
\hline CDDIS & $=$ & Crustal Dynamics Data Information System \\
\hline CMTM & $=$ & Created MATLAB Tools Measurement \\
\hline DGPS & $=$ & Differential Global Positioning System \\
\hline DLR & $=$ & German Aerospace Center \\
\hline DMA & $=$ & Defense Mapping Agency \\
\hline DOD & $=$ & Department of Defense \\
\hline EBE & $=$ & Extended Broadcast Ephemeris \\
\hline ECEF & $=$ & Earth-Centered, Earth-Fixed \\
\hline ECI & $=$ & Earth-Centered Inertial \\
\hline EKF & $=$ & Extended Kalman Filter \\
\hline FCSL & $=$ & Flight Control Systems Laboratory \\
\hline GAST & $=$ & Greenwich Apparent Sidereal Time \\
\hline GLONASS & $=$ & Russion GNSS \\
\hline GNSS & $=$ & Global Navigation Satellite System \\
\hline GPS & $=$ & Global Positioning System \\
\hline GPST & $=$ & Global Positioning System Time \\
\hline GSFC & $=$ & Goddard Space Flight Center \\
\hline GSOC & $=$ & German Space Operations Center \\
\hline HIL & $=$ & Hardware in the Loop \\
\hline IERS & $=$ & International Earth Rotation and Reference System Service \\
\hline ITRF & $=$ & International Terrestrial Reference Frame \\
\hline IGP & $=$ & International GNSS Service Predicted Product Data \\
\hline IGS & $=$ & International GNSS Service \\
\hline IGU & $=$ & International GNSS Service Ultra-rapid Product Data \\
\hline IF & $=$ & Intermediate Frequency \\
\hline INS & $=$ & Inertial Navigation System \\
\hline JPL & $=$ & Jet Propulsion Laboratory \\
\hline LLA & $=$ & Latitude, Longitude, Altitude \\
\hline MEMS & $=$ & Micro-Electro-Mechanical Sensor \\
\hline MCS & $=$ & Master Control Station \\
\hline NAVSTAR & $=$ & NAVigation Satellite Timing And Ranging system \\
\hline NGS & $=$ & National Geodetic Survey \\
\hline SBC & $=$ & Single Board Computer \\
\hline SOPAC & $=$ & Scripps Orbit and Permanent Array Center \\
\hline SP3 & $=$ & Standard Product Version 3 \\
\hline SUAV & $=$ & Small Unmanned Aerial Vehicle \\
\hline SV & $=$ & Space Vehicle \\
\hline PAC & $=$ & Propulsion Assisted Control \\
\hline PPM & $=$ & Pulse Position Modulation \\
\hline PPS & $=$ & Precise Position Code \\
\hline
\end{tabular}




$\begin{array}{lll}\text { PPP } & = & \text { Precise Point Positioning } \\ \text { PPT } & = & \text { Precision Time Protocol } \\ \text { PRN } & = & \text { Pseudo-Random-Noise } \\ \text { PWM } & = & \text { Pulse Width Modulation } \\ \text { RFI } & = & \text { Radio Frequency Interference } \\ \text { USGG2009 } & = & \text { United States Gravimetric Geoid } \\ \text { UCSD } & = & \text { University of California at San Diego } \\ \text { UT1 } & = & \text { Universal Time 1 } \\ \text { UTC } & = & \text { Coordinated Universal Time } \\ \text { UTM } & = & \text { Universal Transverse Mercator } \\ \text { WGS } & = & \text { World Geodetic System } \\ \text { WVU } & = & \text { West Virginia University }\end{array}$




\section{Chapter 1 : Introduction}

\subsection{Problem Description}

The development of precise navigation systems has been an interesting and changing research area in recent years. Advancements in electronics and their decrease in cost have allowed an overall increase in position accuracy and a general increase of interest within GPS positioning for a variety of applications. The first commercial receiver available in 1984 cost $\$ 150,000$ and weighed 100 pounds with an accuracy of 100 meters; today hand-held GPS receivers can cost as little as $\$ 70$ with a much higher accuracy. With these advancements, GPS usage has become an everyday event for many individuals.

Also, since the formation of the International Global Navigation Satellite System Service (IGS) in 1994, there is an availability of precise Global Positioning System (GPS) product data. IGS has a global tracking network of permanent, continuously operating GPS stations. These stations provide the IGS Analysis Centers with datasets, which are analyzed and refined to provide precise products such as satellite ephemerides and clock solutions [1]. The availability of information about the exact location of an object is invaluable in many research areas $[\mathbf{2 , 3 , 4 , 5 ]}$. The use of the IGS product data can provide a real-time geodetic cm-level accuracy for a single frequency GPS receiver [1]. Details on the format of recent IGS products and data is provided and discussed.

The use of a high-level computing environment allows an acceleration of learning, teaching, and research in engineering and sciences [6]. MATLAB has currently been adopted by more than 5000 universities and colleges. The ability to integrate and use IGS products and data in a modern algorithm work environment, such as MATLAB, is limited [7,8,9,10]. Due to these reasons, MATLAB was selected to allow the importation and manipulation of a majority of IGS products and raw data types for their use in a real-time application.

Throughout this graduate project, a number of tools were needed to be developed to properly obtain and use the IGS information in MATLAB. A useful article by Borre discusses the development of the Easy Suite [7]. Easy Suite I \& II are a set of MATLAB tools that can be used in learning the basics of the GPS. Borre's tools consist of useful computational tasks and plotting functions for GPS information. A MATLAB toolbox developed by Dai et al. presents an attitude 
estimation scheme of a rigid platform by means of multiple non-dedicated antennas using GPS [11]. The algorithms developed by Horemuz and Andersson is intended for real-time processing and computes the position and velocity of GPS satellite from both broadcast and precise ephemerides [12]. The algorithms include polynomial interpolations and different order testing. The tools developed for this thesis are oriented in obtaining and using a variety of IGS product data within MATLAB. The tools also include expansions similar to Horemuz and Andersson's algorithms and additional tools for the use of recorded GPS data from a NovAtel OEMV-1. Horemuz and Andersson provided the use of IGS broadcast and precise ephemerides within MATLAB however the created MATLAB tools in this text incorporates global ionospheric error grids as well. As mentioned in Schenewerk [13] and Feng et al. [14], the best interpolator for satellite orbits is a trigonometric function. Therefore, a Fourier series interpolation was used in this project, which provides a sub-centimeter level accuracy.

\subsection{Project Overview}

The goal of this effort was to develop a set of MATLAB tools to allow the manipulation of IGS products and data. The IGS information that is able to be used in MATLAB includes precise ephemerides, satellites clocks, raw ephemerides, and global ionospheric error grids. These integrated together could supplement a precise point positioning (PPP) algorithm. PPP is the state-space solution in the processing of pseudorange and carrier phase measurements from a single GNSS receiver. The IGS information was used to increase the accuracy and understanding of position estimation. For the development and usability of these tools, initial orbit determinations and manipulations of the information are evaluated. These expansions are derived from previous publications; these mathematical calculations are described in detail. Satellite velocity estimates were produced from the IGS broadcast information by a conventional rotation matrix method. Next, interpolation strategies were employed to provide a precise satellite position estimates for the use in a near real-time and real-time application. A comparison is then conducted by using an Extended Kalman filter (EKF) that uses measurement data from recorded GPS information and measurement data that is created from the developed MATLAB tools. A difference between the estimation performances is observed and evaluated to show that the created measurement can improve the estimation. 


\subsection{Literature Review}

Within the literature review, two sections of previous work are discussed. These two sections are the simulation of the global positioning system and the use of product data provided by the International GNSS Service (IGS).

\subsubsection{Simulating the Global Positioning System}

The applications and developments within navigation systems demand an up to date testing and verification means. From experimental testing to hardware-in-the-loop (HIL), simulation is by far the most reasonable and economical method in evaluating an algorithm. A large number of scenarios and quality of measurements can be evaluated through the employment of simulation.

Numerous GPS simulators have been created in a software format $[\mathbf{1 5}, \mathbf{1 6}, 17]$. Other uses for these simulators are for software receiver development [17]. Specific GPS simulators for particular fields, such as space, have been developed due to complexities of their dynamics [18]. HIL simulators facilitate several stages of research and product development, including requirement analysis, design and development, integration, production, maintenance, and support [19]. HIL simulators have been used in many research topics from GPS receiver implementation to navigation algorithm evaluation $[\mathbf{2 0 , 2 1 , 2 2 , 2 3}]$. An advanced GPS hybrid simulator (AGHS) developed by Brown and Gerein was created to address next generation GPS testing issues for the civilian and military markets [24]. This simulator provides digital and radio frequency simulation and incorporates data storage for playback of real-world GPS signals from field tests.

GPS simulators in a software format, compared to HIL, are usually more flexible and economical to conduct a variety of research topics with respect to GPS. Gustavsson created a GPS constellation simulator for navigation algorithm development. This simulator was validated by comparing simulated and calculated hardware code parameters from a GPS receiver using the same input [25]. Abart simulated the GNSS constellations [26], and included a two state statistical model for the local environment based on Boulton [27]. Redmill used a bounded slow time varying bias, constant offset bias, and Gaussian noise for his GPS model [28]. A software intermediate frequency (IF) GNSS signal generator developed by the Position, Location and Navigation (PLAN) group has been proven to be very effective in the development of a software receiver due to its flexibility [29]. 
Alternatively, other approaches have been developed to model errors associated with GPS. Error models are able to be integrated into an established simulation environment just as Rankin had developed. Rankin created a software package that modeled GPS and differential GPS (DGPS). The model produces position errors in a local coordinate system. This software was used in the Real-Time Simulator at the NASA Langley Research Center [30]. Dong created an IF GPS signal simulator, which had multiple error sources which included satellite clock errors, ionosphere and troposphere errors, ephemeris errors, and multipath errors [31]. A frequency domain approach was conducted to determine a GPS error model by using shaping filters by Kong [32]. B.L. Malleswari et al. used a Kalman filter to try and improve the accuracy in locating the GPS receiver by filtering the range measurements with the datum conversion between Universal Transverse Mercator (UTM) and World Geodetic System (WGS - 84) using a single frequency ML-250 hand held GPS receiver [33].

\subsubsection{Using International GNSS Service Product Data}

National geodetic agencies and GPS users interested in geodetic positioning have adopted the IGS precise orbits to achieve cm-level accuracy. There are multiple strategies to increase the precision of a user's position. Kouba describes in detail two post-processing approaches: one uses un-difference dual-frequency pseudorange, and the other uses carrier phase observations. These two strategies use IGS precise orbit products for a stand-alone precise geodetic point positioning with centimeter precision [1]. An interesting article by Yuan et al. proposes aerial triangulation with GPS precise point positioning (PPP) as a way to avoid the use of DGPS reference stations [34]. The GPS PPP kinematic positioning software created by Yuana et al. is called Caravel and uses precise IGS satellite positions and clock products in determining the UAVs precise location. A generic PPP software that estimates the position, clock bias of the user receiver, tropospheric delay parameters, and ionospheric-free ambiguities in the un-differenced carrier phase data was developed by Honda et al [35].

Precise orbit determination strategies also have used IGS product data. Bogdanov et al. developed algorithms for the computation of the navigation satellite orbits in post-processing at a high level of accuracy using IGS position information [36]. Their work stated that the position of a satellite at an arbitrary point of time could be calculated by linear interpolation with a Kalman filter having a $5 \mathrm{~Hz}$ sampling frequency. This interpolation strategy does not exceed a $2 \mathrm{~mm}$ 
error. Another text from Hobbs and Bohn discuss about the two main estimation techniques, the batch least squares approach and the EKF. The results are similar between the two strategies but they give merit to the EKF due to speed, precision, and smoothing [37].

The development of a global ionospheric total electron content mapping using the GPS was discussed in Komjathy's dissertation [38]. Komjathy goes into detail in how he used IGS products to approximate vertical TEC above each IGS station using stochastic parameters in a Kalman filter. Within this dissertation the TEC modeling techniques demonstrated to be viable in providing independently-derived ground-based ionospheric delay corrections for single frequency applications. 


\subsection{Organization}

This thesis is organized as follows; Chapter 2 includes general background information of the United States global positioning system and briefly goes into detail about the following topics within the GPS system: history of GPS, GPS signals, user position determination, reference frames, satellite position determination, GPS error sources, International GNSS Service, GPS/INS Navigation, and the Extended Kalman Filter. Chapter 3 defines the experimental setup and the avionics, including a detailed description of the developments produced by the author. The development of the MATLAB tools is described in Chapter 4. Thereafter, Chapter 5 presents the estimation comparison. Within this chapter, the estimated true position is determined, and the refinement of satellite position from recorded GPS data is discussed. Next within this chapter is the formulation of the EKF used in the evaluation. The last section of this chapter is a comparison between an EKF that uses two different measurement sources: baseline measurements and measurements created from precise orbital positions developed by the MATLAB tools. In Chapter 6 the conclusion and future directions are discussed. 


\section{Chapter 2 : Background Information}

\subsection{Global Positioning System}

Within this section, a brief history of the Global Positioning System (GPS) is provided. Thereafter, general information is presented about the following topics : the history of GPS, GPS signals, how GPS allows a user to estimate their position, commonly used reference frames, and how a GPS satellite's position is calculated, and GPS error sources.

\subsubsection{Global Positioning System History}

The GPS is part of a space based navigation system developed by the United States Department of Defense (DOD) under its NAVSTAR satellite program [39]. GPS was declared fully operational in 1995 after 24 satellites were placed successfully in their respective orbits and successful tests were completed for military use [40]. The structure of GPS has three segments: space, control and user.

The space segment is a baseline constellation consisting of 24 satellites. These satellites each have an orbital period of approximately 12 hours, a radius of 26,560 km and nearly a circular orbit. These satellites are arranged evenly over six orbital planes that are inclined by 55 degrees relative to the equator. Nominally each plane has four satellites, but the constellation can support up to 30 satellites in orbit. The number of working satellites fluctuates due to maintenance. Figure 2.1 from Grewal's textbook is provided to view the structure of the GPS constellation. 


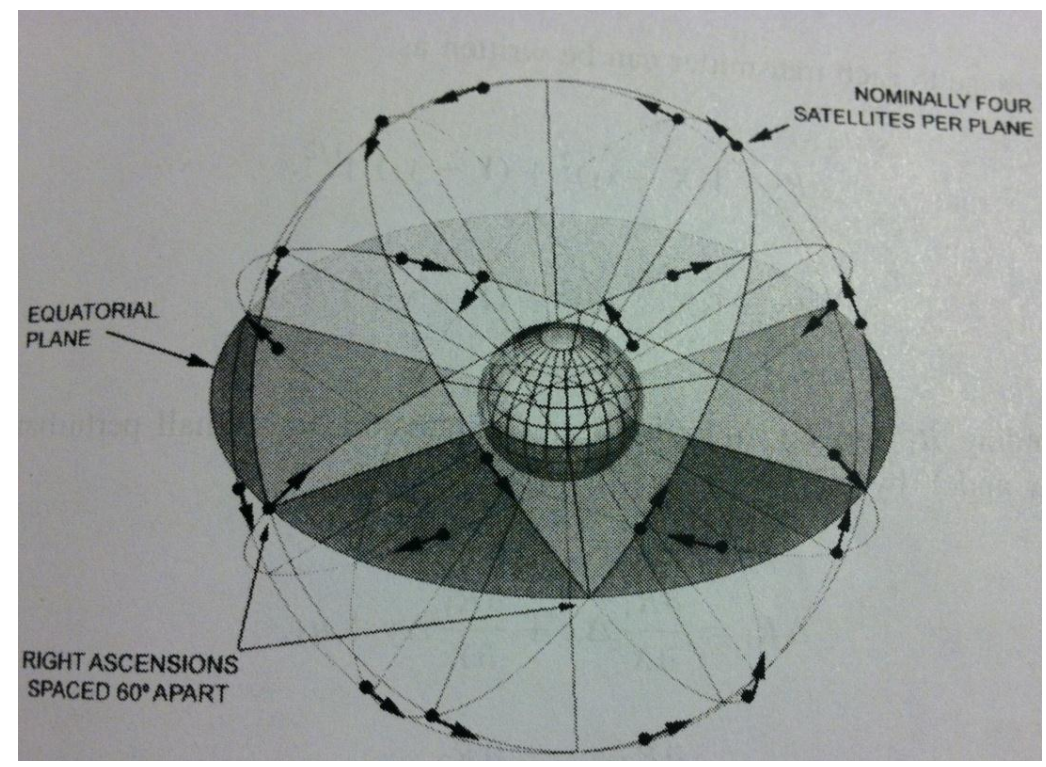

Figure 2.1: GPS Orbit planes [39]

The control segment is primarily a master control station (MCS) located in Colorado which operates the GPS. This station has the command and control functions of the constellation. There are 17 monitoring stations that allow each satellite to be viewed by at least two stations at any time. The responsibility of the control segment is to monitor, maintain the health of satellites and their orbits, and also keep the overall maintenance of the system up to date.

The user segment consists of all the GPS receivers around the world. Through the advancements in integrated circuits, the cost and size of GPS receivers have decreased considerably in the past 20 years. Theoretically, the GPS was designed to allow a user to have three or more GPS satellites always visible from most locations on the Earth [39]. 


\subsubsection{GPS Signals}

At this time, each satellite transmits continuously two spread spectrum signals in the L-band frequency. The Link-1 (L1) has a carrier frequency, $f_{1}=1575.42 \mathrm{MHz}$, and Link-2 (L2) has a carrier frequency, $f_{2}=1227.6 \mathrm{MHz}$. The L1 and L2 frequencies are integral multiples of a base frequency, $f_{0}=1.023 \mathrm{MHz}$, their multiples are as follows: $f_{1}=1540 f_{0}$, and $f_{2}=1200 f_{0}$. The L1 frequency is for civil users and the L2 is for DOD-authorized users. The L1 signal from each satellite uses binary phase-shift keying (BPSK), modulated by two binary codes. These binary codes are also called pseudo-random-noise (PRN) sequences or PRN codes. In this family of PRN codes the standard position code (SPS) is also called the course/acquisition code (C/Acode) and the encrypted code is called the precise position code (PPS), or P-code. The L1 signal is modulated by the C/A-code and P-code in phase quadrature. The L2 signal is modulated only by the P-code.

The PRN codes allow users to access multiple GPS satellite signals on the same carrier frequency. PRN codes are stored and known by a ground GPS receiver. A GPS receiver correlates a GPS signal's PRN code to the stored PRN codes to identify the satellite. For each satellite the C/A-code is a unique sequence of 1023 bits and repeated at every millisecond. The P-code has a length of 259 days, during which each satellite transmits a unique portion of the Pcode. Each satellite has a portion of exactly one week of the P-code before it repeats. The GPS signal also transmits the navigation data, which is also called the navigation message. This binary-coded message includes satellite health, orbital parameters, and clock bias parameters. Section 2.1.5 discusses in more detail the information within the navigation message. 


\subsubsection{User Position Determination}

The GPS space, control, and user segments work together and use the idea of trilateration called time-of-arrival to determine the position of the receiver. Time-of-arrival uses the speed of a radio wave and the transmit time from which it was transmitted and received to calculate the distance between the transmitter and receiver. By knowing three transmitter locations and the distances between them and the receiver, the receiver's position can be determined. In order to measure the transit time of the signal the clock of the transmitter and receiver need to be synchronized. If this synchronization is off by $1 \mu \mathrm{s}$, an error of 300 meters can be accrued. This is due to the fact that the radio signals are moving approximately at the speed of light.

The transmitters in the case of GPS are the satellites which are moving at rate of $4 \mathrm{~km} / \mathrm{s}$. The position of these satellites can be approximated one to two days in advance with an error of a few meters. The positions of the satellites are computed using ephemeris data broadcasted by the satellites. Ephemeris data that is broadcasted are accurate within 5 to 10 meters. However, there are instances when a user receiver may make an anomalous interpretation of the broadcast signal [41]. Determining GPS satellite locations is discussed further in Section 2.1.4.

The transit time is sent within the signals, and their accuracy is nearly perfect due to the synchronized atomic clocks aboard the satellites. This synchronization of time between all satellites represents the GPS Time (GPST). However, due to using an inexpensive quartz oscillator, the measurement of the transit time from a GPS receiver is biased for all satellites equally. Therefore this causes the measured ranges between satellites to be biased by a common amount [42]. These measured ranges are called pseudoranges due to this ambiguity. To further explain pseudoranges the following figure and equations from Kaplan's textbook are used [43].

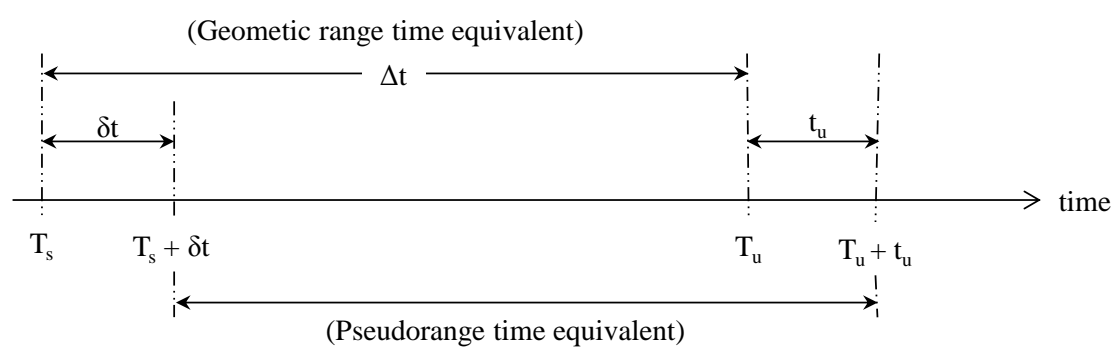

Figure 2.2: Range measurement timing relationships [43] • 
In Figure 2.2 the timing relationship between receiver and satellites are shown. The variables are defined as such: $T_{s}$ is the system time at which the signal left the satellites, $T_{u}$ is the system time at which the signal reached the user receiver, $t_{u}$ is the offset of the receiver clock from the GPST, and $\delta t$ is the offset of the satellite clock from GPST. The satellite clock reading at the time that the signal left the satellite would be $T_{s}+\delta t$ and the user receiver clock reading at the time the signal reached the user receiver would be $T_{u}+t_{u}$. The geometric range, $r$, can be determined with $r=c\left(T_{u}-T_{s}\right)=c \Delta t$ [43], where $c$ is the speed of light. By understanding Figure 2.2, the pseudorange, $\rho$, can be calculated by $\rho=r+c\left(t_{u}-\delta t\right)$ [43]. The $\delta t$ is actually modeled within the GPS signal by a bias, drift and drift rate, $a_{f 0}, a_{f 1}, a_{f 2}$, therefore the only unknown is, $t_{u}$, the offset of the receiver clock from the GPST. To determine the position of a user receiver it has been stated that three satellites need to be used, but due to the inexpensive design of a GPS receiver a fourth satellite is required so that the time offset $t_{u}$ can be determined. 


\subsubsection{Reference Frames}

In GPS there are multiple coordinate and time systems which are explained in this section. For a precise measurement, a precise coordinate system is essential [42]. For convenience and due to the position of the user rotating with the Earth, the coordinate system is chosen to also rotate with the Earth. This selected coordinate system is called Earth-centered, Earth-fixed (ECEF) and has its center at the center of mass of the Earth. A user's receiver usually displays its position in ellipsoidal coordinates: latitude, $\phi$, longitude, $\lambda$, altitude, $h$, or LLA. These parameters can be calculated from Cartesian coordinates in the ECEF system. This transformation is done by an iterative method, for more information see Kaplan Section 2.2.3 [43] or Misra Appendix 4.A [42]. The transformation from LLA to ECEF can be done using

$$
\left[\begin{array}{c}
x_{E C E F} \\
y_{E C E F} \\
z_{E C E F}
\end{array}\right]=\left[\begin{array}{c}
\frac{a \cos (\lambda)}{\sqrt{1+\left(1-e^{2}\right) \tan ^{2}(\phi)}}+h \cos (\lambda) \cos (\phi) \\
\frac{a \sin (\lambda)}{\sqrt{1+\left(1-e^{2}\right) \tan ^{2}(\phi)}}+h \sin (\lambda) \cos (\phi) \\
\frac{a\left(1-e^{2}\right) \sin (\phi)}{\sqrt{\left(1-e^{2}\right) \sin ^{2}(\phi)}}+h \sin (\phi)
\end{array}\right]
$$

where $e$ is the eccentricity of the Earth ellipsoid. The type of conic section the orbit has derives the eccentricity. Due to the shape of the Earth, the major axis, $a$, is the mean equatorial diameter of the Earth [44].

The standard model of the Earth in terms of the global positioning system is the World Geodetic System 1984 (WGS84). The WGS84 is an ECEF frame, and is defined/maintained by the United States National Geospatial-Intelligence Agency (NGA). Not all scientific communities, however, use the WGS84 coordinate frame. The International Terrestrial Reference Frame (ITRF) is another ECEF frame, and is defined by the International Earth Rotation and Reference Systems Service (IERS). When discussing GPS, ITRF is not the usual coordinate frame in which a user's position is defined. The differences between the ITRF and WGS84 frame are discussed in Section 2.2. 
The WGS84 includes a detailed model of the Earth including: gravitational irregularities, a model of its ellipsoidal shape, and a geoid for a reference to measure height. In most instances, the concept of height is referenced to the global mean sea level. This surface is a reference to the geoid. The gravitational irregularities are a natural feature of the Earth and are not easily modeled; therefore modeling is done by spherical harmonic coefficients. Newer versions of Earth's gravity model are developed continuously. The United States Gravimetric Geoid of 2009 (USGG2009) is based on a 1'x1' gravity grid, for more information on the USGG2009 refer to Wang [45].

As described in Section 2.1.3, to determine the position of a user receiver, reference positions of satellites need to be known. The satellites are referenced in an Earth-Centered inertial (ECI) coordinate system due to convenient notation of orbital motion in a non-rotating frame. The origin of ECI is the center of mass of the Earth, the $x y$-plane coincides with the Earth's equatorial plane, the $+x$-axis points to the vernal equinox direction (fixed celestial direction), the $+z$-axis is normal to the $x y$-plane in the direction of the North pole, and the $+y$-axis is chosen such that it is a right handed system [42]. Satellites broadcast their position in Keplerian orbital parameters. To determine an orbit's orientation to the ECEF frame, the inclination angle, $i$, longitude of ascending node, $\Omega$, and the argument of perigee, $\omega$, are needed. These are described with Figure 2.3-1 in Bate's textbook [44] or the provided Grewal's textbook Figure 2.10:

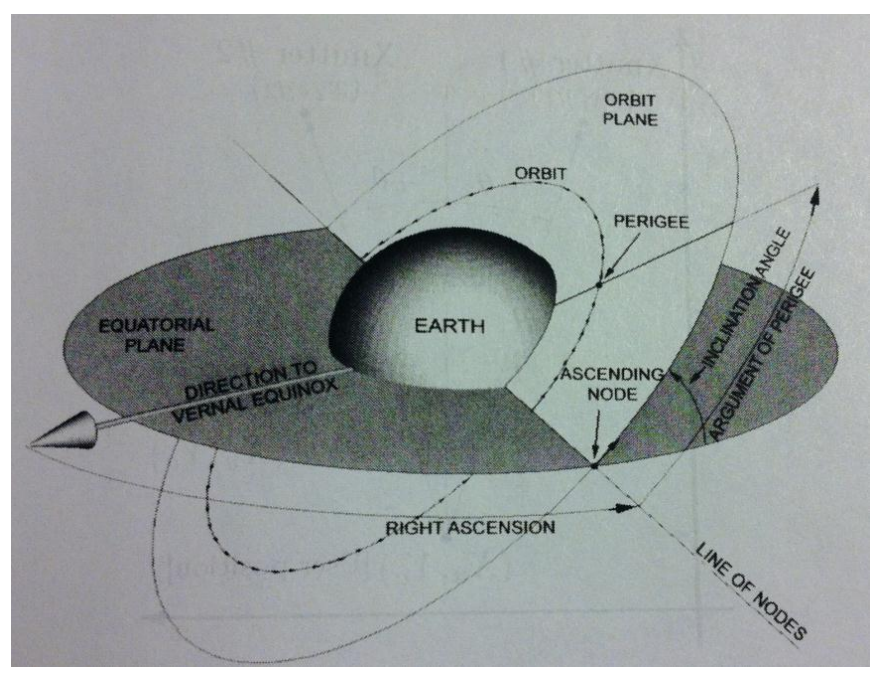

Figure 2.3: Satellite geometry with parameters labeled Figure 6.2 [39] 
There are multiple timescales due to the non-uniform spin rate of the Earth. Universal Time 1 (UT1) is an astronomical definition of time, determined by measurements of observatories and corrected for their locations on Earth. This makes UT1 not a uniform timescale. Coordinated Universal Time (UTC) is the time scale throughout the world. UTC is a product of the International Earth Rotation and Reference System Service (IERS). This timescale is derived from a cesium atom, however IERS steers UTC such that the difference between UTC and UT1 does not exceed 0.9 seconds [46].

As stated previously, GPST is the timescale that is used in the GPS network. This is derived from a composite of clocks that consist of all clocks in the GPS network, i.e. monitor station and satellites. The GPS control segment is required to steer the GPST within $1 \mu$ s of UTC. GPST is defined by the number of seconds that have passed since Saturday midnight and the GPS week. The GPS week is incremented by 1 each week from which it started at 0 on the midnight January 5, 1980 GPST. The GPS week number field is a modulo 1024, which means at the end of week 1023 it will roll back to 0. This rollback took place at midnight of August 21, 1999 GPST [47]. The conversion from GPST to UTC is explained in Section 2.4.3 of Grewal [39] or Section 5.14 of Tsui $[48]$. 


\subsubsection{Satellite Position Determination}

Idealizing the shape of an orbit, the semimajor axis, $a$, and eccentricity, $e$, are needed to define the ellipse. For the position of the satellite on the ellipse, the only parameter needed is the true anomaly, $v$, which is the angle measured counter-clockwise in the plane of the ellipse between the location of the satellite at a particular time, or epoch, usually denoted by $t_{o}$, and perigee of the orbit. The true anomaly is a function of the eccentric anomaly, $E$, and mean anomaly, $M$. Calculating the true anomaly is discussed further. Due to the Earth not being uniform in shape or density, the satellite orbits are perturbed. These perturbations alter the satellite orbits by rotating them about the orbital plane and the line of ascending nodes in the inertial frame. Also, due to the equatorial bulge, the satellites speed up due to a higher gravity field near the equator, and slow down when moving farther away. This causes the true anomaly to not have a straight forward calculation. The perturbations also require an expansion in the Keplerian parameters. These added parameters include the rate of change of the inclination angle, $d i / d t$, rate of change of the longitude of ascending node, $d \Omega / d t$, the mean motion correction, $\Delta n$, and an amplitude terms for sine and cosine corrections of argument of latitude, orbital radius, inclination angle, respectively denoted as follows: $C_{u s}, C_{u c}, C_{r s}, C_{r c}, C_{i s}, C_{i c}$. The argument of latitude, $\Phi$, is the angle between the satellite and the equator in the orbital plane or the summation of the argument of perigee and true anomaly. Due to the linear change in mean motion through time, the mean anomaly will change as well.

Each GPS satellite broadcasts continuously a navigation data message. This message is sent at every minute or half-minute according to the satellites clock. It takes 30 seconds to transmit a frame which is 1500 bits long. Each frame is evenly divided in length and in sending time into five subframes. Subframe 1 contains the GPS week number, space vehicle (SV) accuracy and health, and satellite clock corrections. Subframes 2-3 have ephemeris data which contain the parameters within Table 2.1. 
Table 2.1: GPS ephemeris parameters in Subframe 2-3

\begin{tabular}{ccc}
\hline Parameter & Description & Units \\
\hline$t_{o e}$ & Ephemeris & $\mathrm{S}$ \\
$\sqrt{a}$ & reference time (second of the GPS week) & $\sqrt{\mathrm{m}}$ \\
$e$ & Square root of semimajor axis & - \\
$i_{o}$ & Argument of perigee & $\mathrm{rad}$ \\
$d i / d t$ & Inclination angle at reference time & $\mathrm{rad}$ \\
$\Omega_{o}$ & Rate of change of inclination angle & $\mathrm{rad} / \mathrm{s}$ \\
$d \Omega / d t$ & Longitude of ascending node of orbital plane at weekly epoch & $\mathrm{rad}$ \\
$M_{o}$ & Rate of change of longitude of ascending node & $\mathrm{rad} / \mathrm{s}$ \\
$\Delta n$ & Mean anomaly at reference time & $\mathrm{rad}$ \\
$C_{u c}$ & Mean motion correction & $\mathrm{rad} / \mathrm{s}$ \\
$C_{u s}$ & Amplitude of cosine correction to argument of latitude & $\mathrm{rad}$ \\
$C_{i c}$ & Amplitude of sine correction to argument of latitude & $\mathrm{rad}$ \\
$C_{i s}$ & Amplitude of cosine correction to inclination angle & $\mathrm{rad}$ \\
$C_{r c}$ & Amplitude of sine correction to inclination angle & $\mathrm{rad}$ \\
$C_{r s}$ & Amplitude of cosine correction to orbital radius & $\mathrm{m}$
\end{tabular}

Subframe 4 includes almanac and health data for satellites 25-32, special messages, satellite configuration flags, and ionospheric and UTC data. Subframe 5 includes almanac and health data for satellites 1-24 and almanac reference time and week number [49]. Subframes 1-3 are sent every 0.5 minutes and subframes $4-5$ are sent every 12.5 minutes. Subframes 1-3 typically change on the hour but can repeat for as long as two hours.

The almanac is a coarse version of ephemerides of all satellites in the constellation. This information allows the user receiver to know what satellites should be in view and when other satellites could become in view with respect to an approximate location of the receiver. This allows the almanac parameters to not be as accurate as the ephemeris parameters [42]. 
From the ephemeris parameters within the navigation message, the following steps need to be taken to calculate a satellite's position:

Step 1 : Determine the corrected mean motion with: $n=\sqrt{\mu / a^{3}}+\Delta n$. In this equation $\mu$ is the geocentric gravitational constant, for its value see Appendix A.1 Relevant Constants Used.

Step 2 : Initially we need to assume that the satellite time or space vehicle time, $t_{s v}$, is also the GPST. Therefore the time of interest, $t$, will equal the space vehicle time, $t_{s v}$. The time since the ephemeris epoch will then be calculated as follows, where $t_{o e}$ is the ephemeris reference time.

$$
t_{k}=t-t_{o e}
$$

Step 3 : Determine the mean anomaly, $M_{k}$, with $M_{k}=M_{0}+n t_{k}$, all other parameters are known from ephemeris data in navigation message.

Step 4 : Use an iterative method to solve Kepler's equation (Equation 2.3) for the eccentric anomaly, $E_{k}$. The most widely used method is the Newton-Raphson. For more information on the implementation of this method see the following sources Misra Section 3.2.2.5 [42] or Bate Section 4.6.4 [44].

$$
M_{k}=E_{k}-e \sin E_{k}
$$

Step 5 : In Step 1 the assumption that the satellite time is the GPST is not correct due to the perturbations acting on the satellite. This requires a relativistic correction term, $\Delta t_{r}$, this term is needed since the satellite antenna phase center is sensitive to small perturbations in most ephemeral parameters [50]. Before calculating the relativistic correction term a constant, $F$, will need to be determined by $F=-2 \sqrt{\mu} / c^{2}$, where $c$ is the speed of light, and $\mu$ is the geocentric gravitational constant. See Appendix A for the value of $c$. Calculating the relativistic correction term with $\Delta t_{r}=\mathrm{Fe} \sqrt{a} \sin \left(E_{k}\right)$. Within in this equation the variables are the following: constant $F$, the orbits eccentricity, $e$, and the square root of the semimajor axis, $a$. 
Step 6 : Equation 2.4 is the correction between the perceived GPST of the satellite and the actual GPST. The $t_{s v}$ in the following equation can be interpreted as the time at which the GPS satellite believes the time to be. The parameters $t_{o c}, a_{f 0}, a_{f 1}$, and $a_{f 2}$ are the clock data reference time and the polynomial clock correction coefficients.

$$
t_{G P S}=t_{s v}-a_{f o}-a_{f 1}\left(t_{G P S}-t_{o c}\right)+a_{f 2}\left(t_{G P S}-t_{o c}\right)^{2}+\Delta t_{r}
$$

Step 7 : Repeat steps 2-4 and in Step 2 the time of interest, $t$, will now be the corrected satellite time, $t_{G P S}$. The new values of $M_{k}, E_{k}$, and $t_{k}$, will replace the previously calculated estimates.

Step 8 : The true anomaly, $v_{k}$, will then be calculated by Equation 2.5. This will be used instead of the conventional equation to eliminate ambiguity through the use of MATLAB®'s ATAN2 function [51].

$$
v_{k}=\tan ^{-1}\left(\frac{\sqrt{1-e^{2}} \sin E_{k}}{\cos E_{k}-e}\right)
$$

Step 9 : The argument of latitude, $\phi_{k}$, will then be calculated with $\phi_{k}=v_{k}+\omega$.

Step 10 : The argument of latitude correction, $\delta \phi_{k}$, the inclination angle correction, $\delta i_{k}$, and radius correction, $\delta r_{k}$, will be calculated with the following three equations.

$$
\begin{array}{rlr}
\delta \phi_{k} & =C_{u s} \sin \left(2 \phi_{k}\right)+C_{u c} \cos \left(2 \phi_{k}\right) & \mathbf{2 . 6} \\
\delta r_{k} & =C_{r s} \sin \left(2 \phi_{k}\right)+C_{r c} \cos \left(2 \phi_{k}\right) & \mathbf{2 . 7} \\
\delta i_{k} & =C_{i s} \sin \left(2 \phi_{k}\right)+C_{i c} \cos \left(2 \phi_{k}\right) & \mathbf{2 . 8}
\end{array}
$$

Step 11 : To obtain the corrected argument of latitude, $u_{k}$, corrected inclination angle, $i_{k}$, corrected radius, $r_{k}$, and corrected longitude of node, $\Omega_{k}$, the following equations will be used. The value of the Earth's rotation rate, $d \Omega_{e} / d t$, can be obtained in Appendix A. 


$$
\begin{gathered}
u_{k}=\phi_{k}+\delta \phi_{k} \\
r_{k}=a\left(1-e \cos E_{k}\right)+\delta r_{k} \\
i_{k}=i_{o}+\frac{d i}{d t} t_{k}+\delta i_{k} \\
\Omega_{k}=\Omega_{o}+\left(\frac{d \Omega}{d t}-\frac{d \Omega_{e}}{d t}\right) t_{k}+\left(\frac{d \Omega_{e}}{d t}\right) t_{o e}
\end{gathered}
$$

Step 12 : The in-plane $x$ and $y$ position of the satellite can be calculated by $x_{p}=r_{k} \cos u_{k}$ and $y_{p}=r_{k} \sin u_{k}$, respectively.

Step 13 : The ECEF position of the satellite can now be calculated by the following equations:

$$
\begin{gathered}
x_{k}=x_{p} \cos \Omega_{k}-y_{p} \cos i_{k} \sin \Omega_{k} \\
y_{k}=x_{p} \cos \Omega_{k}-y_{p} \cos i_{k} \sin \Omega_{k} \\
z_{k}=y_{p} \sin i_{k}
\end{gathered}
$$

For more information about the process in determining the satellite's position in the ECEF coordinate system, see the following references: Section 3.2.2 [39], Section 2.3 [43], [51], [52].

\subsubsection{GPS Error Sources}

This section is an introduction of all major error sources of GPS and how they can be mitigated. The contributing sources that degrade the performance of a precise and accurate GPS position solution include: Earth's atmosphere, satellite clock and orbit errors, geometry of the constellation, radio frequency interference, multipath signals, and receiver clock error.

The Earth's atmosphere has two sections that disturb radio signals that pass through it. The ionosphere and troposphere are the error building sections of the atmosphere. These sections of the atmosphere cause errors in GPS. The ionosphere is a portion of the upper atmosphere layer and extends approximately from 50 to $1000 \mathrm{~km}$ above the earth. The atmosphere is so thin here that free electrons can exist for short periods of time. These free electrons cause radio waves to slow down when passing through this layer. This delay in time correlates to an assumed increase in distance which can cause erroneous GPS measurements. This layer is ionized because of the 
suns radiation. A dual frequency GPS receiver can estimate these errors since the delay time is a function of the frequency of the signal. For single frequency GPS receivers the ionospheric error is the largest error source. Single frequency GPS receivers use an estimate the ionospheric error through the Klobuchar's Model. The Klobuchar model is a compromise between complexity and accuracy. This model is said to be capable of correcting up to $70 \%$ of the ionosphere error. Coefficients of this model are within the broadcasted navigation message.

The lowest portion of the Earth's atmosphere, the troposphere, approximately contains $80 \%$ of the atmosphere's mass and $99 \%$ of its water vapor [53]. The troposphere is $80 \%$ saturated [54], which causes radio signals to refract. This refraction effects the GPS signal propagation by retarding and bending it. This causes mitigation of this sort of error very difficult due to the spatial and temporal variation in water vapor within the atmosphere. There is also a dry gascomponent that can be modeled by using temperature, pressure and humidity as well as the propagation path to determine its refractivity profile [39].

The geometry of the GPS constellation dictates the precision of a GPS receiver's solution. By looking at a simple two-dimensional example more insight is obtained. In Figure 2.4, a user measures their distance from a pair of stations (S1 and S2) at known locations. If perfect range measurements were available, the intersection of two circles centered at S1 and S2 with the radius of the range measurement would be the exact location of the user.
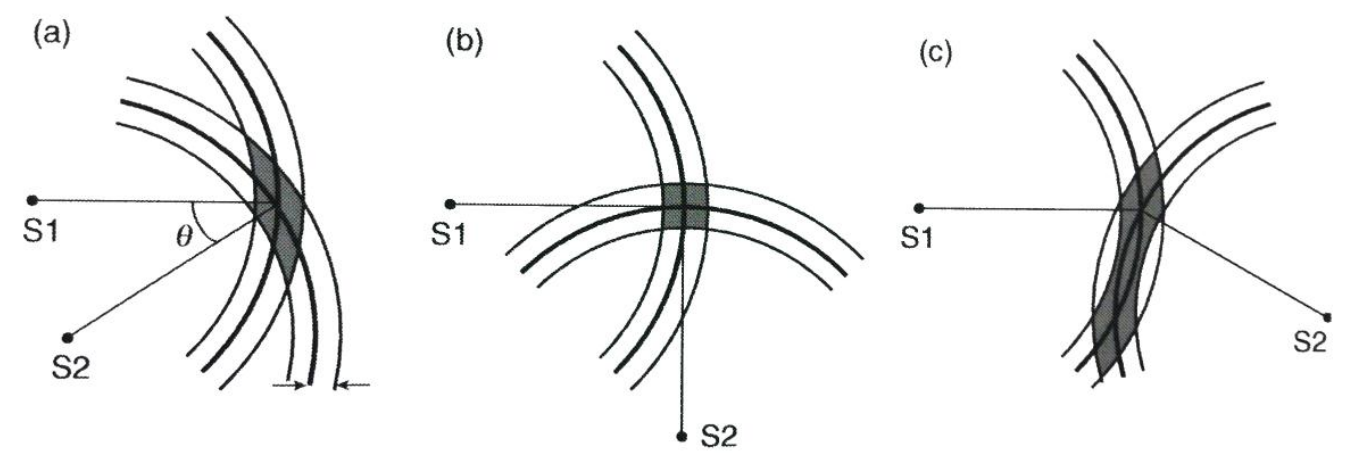

Figure 2.4: 2-Dimension example of how GPS geometry affects position measurement [42] 
Imperfect range measurements, however, have an associated uncertainty. By looking at the figure above the geometric arrangement of stations reveals a change in the region of uncertainty. For the acute and obtuse angle cases (a) and (c) these regions are much larger than case (b) [42].

GPS signals that are received by a GPS receiver are very weak. The radio frequency power at the antenna input port of a satellite is only approximately 50 Watts. This makes the GPS signal susceptible to unintentional interference or intentional jamming. A common misconception associated with GPS is that the GPS signals go directly from the GPS satellite to the user. Reflections of GPS signals are likely when there are obstructions near the GPS antenna. The reflected GPS signal causes an increase in the propagation time. From Section 2.1.3 an increase in propagation time will result in an increase in pseudorange, resulting in possible erroneous measurements. The multipath propagation is a dominant source of error for differential GPS systems (DGPS). DGPS is a technique in which a reference station at a known location updates a user's receiver with estimated error corrections in real-time. There are two primary methods of multipath mitigation: spatial processing and time-domain processing. The spatial processing determines the most likely signal propagation geometry to isolate the direct-path received signals. The time-domain process determines the direct-path received signals through the use of the corrupted multipath signal within the receiver, Section 5.6 [39].

Other errors arise with discrepancies between ephemeris data. This was discussed in Jefferson and Bar-Sever's article in which different GPS receivers on the ground were reporting different broadcast messages. These discrepancies caused orbit differences as large as 300 meters. They concluded that the broadcast GPS orbit compared to a precise IGS orbit product was accurate at the 5- to 10- meter level. However, there are occasions when the GPS receiver may make an anomalous interpretation of the broadcast signal [41].

As stated previously, pseudorange measurements are very sensitive to GPS receiver clock offset errors. These are due to the inexpensive quartz oscillator within most GPS receivers. With the inclusion of a $4^{\text {th }}$ satellite, this time offset can be estimated through Kalman filtering. There are numerous examples of this throughout history. A recent example from the Deutsches Zentrum fur Luft-und Raumfahrt (DLR)/German Space Operations Center (GSOC) precisely estimated the orbit of the MetOp-A-satellite by the clock products generated by their Kalman filter [55]. Shmaliy et al. presented a study of different types of optimal and quasi-optimal Kalman filters based on crystal and rubidium oscillators using reference timing from a GPS 
receiver [56]. Within this article, a crystal clock was investigated in detail in terms of the time interval error, Allan deviation and precision time protocol (PTP) variance [57]. The experimental information within the above reference characterizes a GPS receiver clock. Misra and Enge's textbook also includes information about the stability of oscillators within Section 4.2.3 [42]. 


\subsection{International GNSS Service (IGS)}

A brief description about IGS and the products/data that are available from this organization is given in this section. The IGS was founded in 1994, and is a voluntary federation of more than 200 worldwide national agencies, universities, and research institutions [58]. This federation creates a global network of over 350 permanent, continuously operating, geodetic-quality United States GNSS (GPS) and Russian GNSS (GLONASS) tracking sites. IGS collects, archives, and distributes GPS and GLONASS observation datasets. These datasets are also analyzed and refined to form IGS products.

The archiving of station data is completed at three Global Data Centers and six Regional Data Centers. The global data centers, the Crustal Dynamics Data Information System (CDDIS), Scripps Orbit and Permanent Array Center (SOPAC), and the National Geographic Institute (IGN) which are located respectively at NASA's Goddard Space Flight Center (GSFC), the University of California at San Diego (UCSD), and Paris, France. There are a total of ten analysis centers, which regularly process the data and contribute to the formation of the official IGS combined products. The IGS International Governing Board sets policies that dictate the Central Bureau in its management of IGS [59].

IGS products support a wide variety of scientific areas such as the following: the improvement and extension of the ITRF, monitoring deformations of the solid Earth and variations in the liquid Earth, variations in the Earth's rotation, determination of orbits of scientific satellites, and monitoring the troposphere and ionosphere [60]. For more information specifically about IGS and the direction it is currently striving towards view following references [61], [62]. 
The table below is the current types of satellite products and their respective attributes that are available from IGS (Broadcast data is included in this table for comparison reasons):

Table 2.2: IGS Orbit product accuracy table [60]

\begin{tabular}{|c|c|c|c|c|c|}
\hline \multicolumn{2}{|c|}{$\begin{array}{l}\text { GPS Satellite Ephemerides/ } \\
\text { Satellite \& Station Clocks }\end{array}$} & Accuracy & Latency & Updates & Sample Interval \\
\hline \multirow{3}{*}{ Broadcast } & orbits & $\sim 100 \mathrm{~cm}$ & \multirow{3}{*}{ real time } & & \multirow{3}{*}{ daily } \\
\hline & \multirow{2}{*}{ Sat. clocks } & $\sim 5 \mathrm{~ns} \quad \mathrm{RMS}$ & & & \\
\hline & & $\sim 2.5 \mathrm{~ns}$ SDev & & & \\
\hline \multirow{3}{*}{$\begin{array}{l}\text { Ultra-Rapid } \\
\text { (predicted half) }\end{array}$} & orbits & $\sim 5 \mathrm{~cm}$ & \multirow{3}{*}{ real time } & \multirow{3}{*}{$4 \mathrm{x}$ daily } & \multirow{3}{*}{$15 \mathrm{~min}$} \\
\hline & Sat locke & $\sim 3$ ns $\quad$ RMS & & & \\
\hline & Sat. clocks & $\sim 1.5 \mathrm{~ns}$ SDev & & & \\
\hline \multirow{3}{*}{$\begin{array}{l}\text { Ultra-Rapid } \\
\text { (observed half) }\end{array}$} & orbits & $\sim 3 \mathrm{~cm}$ & \multirow{3}{*}{3 hours } & \multirow{3}{*}{$4 \mathrm{x}$ daily } & \multirow{3}{*}{$15 \mathrm{~min}$} \\
\hline & \multirow{2}{*}{ Sat. clocks } & $\sim 150$ ps RMS & & & \\
\hline & & $\sim 50$ ps $\quad$ SDev & & & \\
\hline \multirow{3}{*}{ Rapid } & orbits & $\sim 2.5 \mathrm{~cm}$ & \multirow{3}{*}{17 hours } & \multirow{3}{*}{ daily } & $15 \mathrm{~min}$ \\
\hline & & $\sim 75$ ps RMS & & & $5 \mathrm{~min}$ \\
\hline & Sat. \& Stn. clocks & $\sim 25$ ps SDev & & & $3 \mathrm{~min}$ \\
\hline \multirow{3}{*}{ Final } & orbits & $\sim 2.5 \mathrm{~cm}$ & \multirow{3}{*}{$\sim 13$ days } & \multirow{3}{*}{ weekly } & $15 \mathrm{~min}$ \\
\hline & \multirow{2}{*}{ Sat. \& Stn. clocks } & $\sim 75$ ps RMS & & & Sat.: $30 \mathrm{~s}$ \\
\hline & & $\sim 20$ ps SDev & & & Stn.: $5 \mathrm{~min}$ \\
\hline
\end{tabular}

This table clearly reveals the variety and precision of these products over the broadcast data. The newest type of product data is the IGS Ultra-Rapid (IGU) which replaced the IGS Predicted (IGP) in 2000. The main differences between the types of IGS products are the period of latency and the extent of the tracking network used in their computations [1]. The coordinate frame in which the satellite positions are defined is an ITRF. According to a study by Merrigan et al. the ITRF2000 and the current WGS84 are essentially identical [63].

The IGS products have increased in precision and accuracy since there availability. Figure 2.5 was obtained from the National Geodetic Survey website for predicted orbit comparisons. The plot on the left is the weighted root mean square error and the right shows the median differences (mm) of the IGS combined orbit predictions with respect to the IGS Rapid (IGR) 
products. The $x$-axis is from the GPS week 1100, which occurred in February, 2001, and stops approximately at November, 2011.
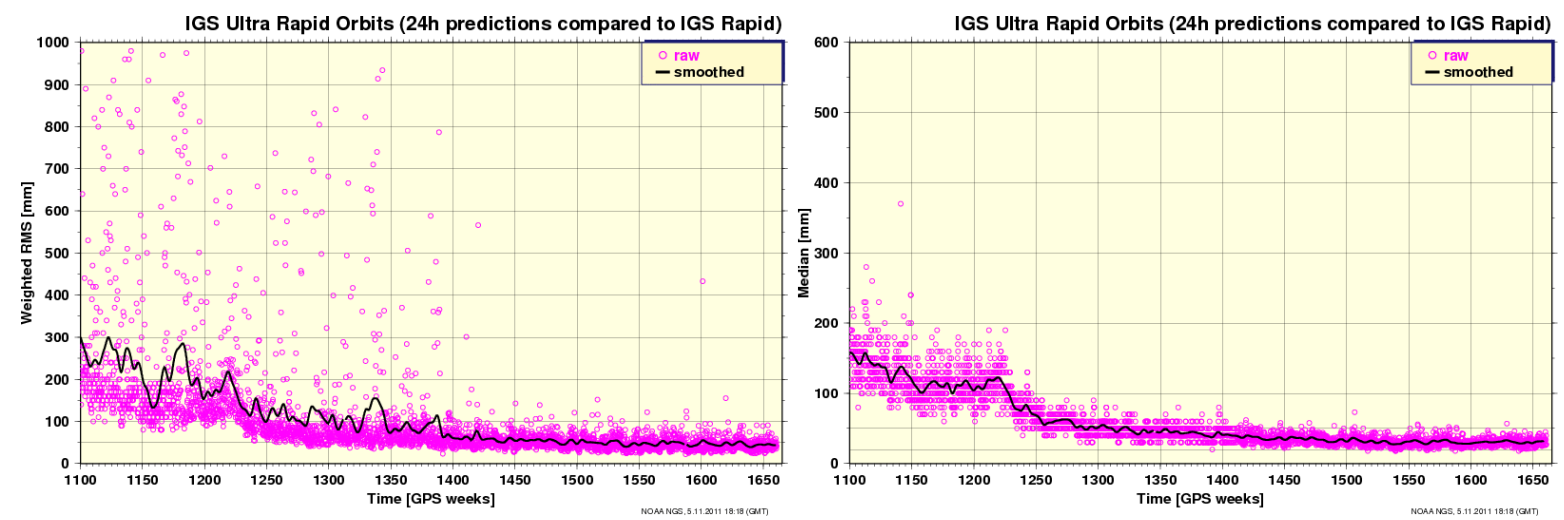

Figure 2.5: Combined IGU Orbits compared to IGR [64]

The above figures reveal the increase in accuracy and precision of the predicted IGU Orbits. The figure below shows a quantitative comparison of the predictions over 24 hours between the median of the IGU combined orbits to IGR in the recent weeks.

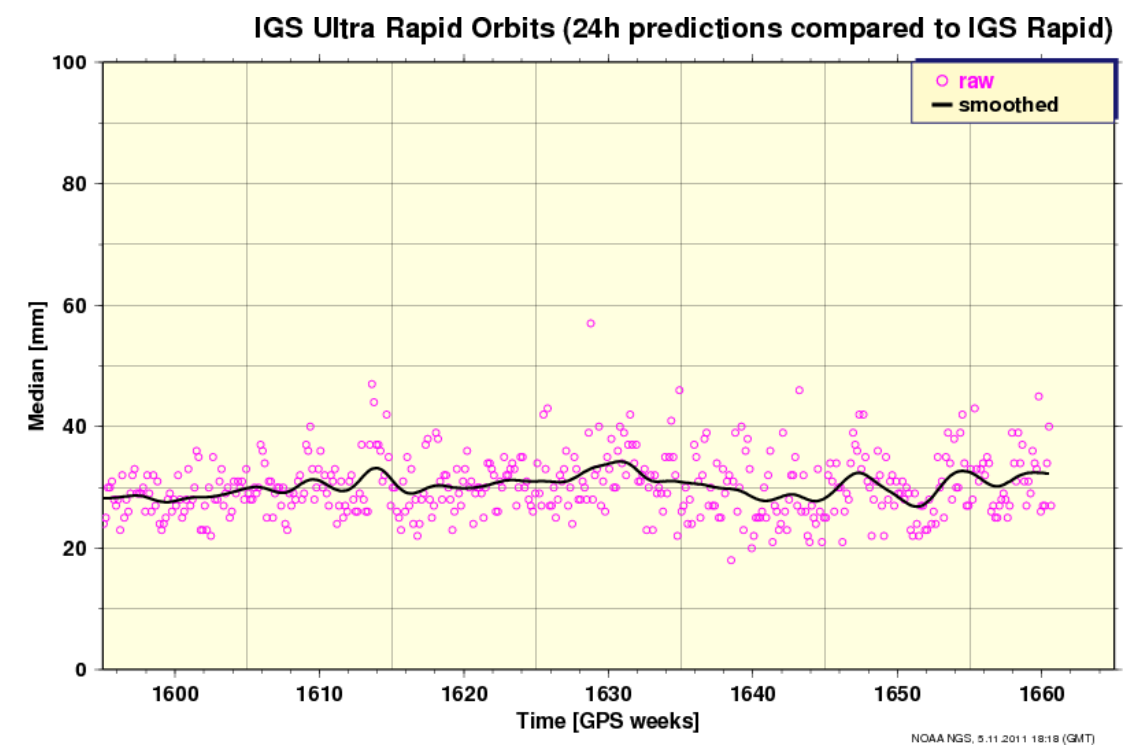

Figure 2.6: Recent week median difference between 24 hr IGU and IGS products [64] 
The intent for the IGU product data is to be used in real-time. For many applications, the differences above are acceptable. Further information is available about the satellite position estimates; however this is discussed later in this thesis.

\subsection{GPS/INS Navigation}

GPS/INS navigation refers to the use of GPS to correct or calibrate a solution from an inertial navigation system (INS). This allows for an accurate navigation solution to be obtained using measurements from low cost sensors. The evaluation of a GPS/INS algorithm is usually conducted by the verification of estimation accuracy. The verification process however can be difficult to impossible due to the lack of a state measurement or cost of a tactical grade sensor.

A GPS solution uses an external reference frame, the constellation of satellites, which allows susceptibility of signal interference, satellite signal blockage, etc. GPS, when a position solution is available, has a bounded error. In contrast, an INS solution is internally referenced, making it not susceptible to external interference. An INS solution has a slow rate of change but has an unbounded error. These two types of navigation complement each other greatly. Usually INS sensors have a very high update rate and typically small short term errors. The integration of GPS and inertial sensors is typically done by a Kalman Filter (KF). The integration of GPS and INS information began in the early 1980s.

The KF was developed in 1960, although it has roots as far back as Karl Gauss in 1795. The Kalman filter was published in 1961 by Kalman and Bucy [65]. Schmidt was accredited to be the first to use KF in application. This KF was for navigation trajectory estimation for the Apollo program [66]. The KF was also derived to recursively minimize a cost function; this is the minimization of the means square error of the estimates. This filter has the ability to estimate past, present and future states. Some example of states could be velocity and position, or attitude orientation. This estimation process can be done without knowing the exact model in which it is estimating. For this filter to be optimal the largest assumption is that the system is linear. This is not true in most applications therefore nonlinear estimation must be considered. 


\subsection{Extended Kalman Filter}

Shortly after the induction of the KF, the Extended Kalman Filter (EKF) was developed [65]. The EKF uses the same framework as the KF, but linearizes the system model around the previous state estimates with a first order Taylor Series expansion. The EKF is considered the standard nonlinear estimator for navigation systems and GPS [67]. The equations for the EKF are given by $[\mathbf{6 6}]$ :

$$
\begin{gathered}
\hat{x}_{k \mid k-1}=f\left(\hat{x}_{k-1}, u_{k-1}, 0\right) \\
P_{k \mid k-1}=A_{k-1} P_{k-1} A_{k-1}^{T}+Q_{k-1} \\
K_{k}=P_{k \mid k-1} H_{k}^{T}\left(H_{k} P_{k \mid k-1} H_{k}^{T}+R_{k}\right)^{-1} \\
\hat{x}_{k}=\hat{x}_{k \mid k-1}+K_{k}\left[z_{k}-h\left(\hat{x}_{k \mid k-1}, 0\right)\right] \\
P_{k}=\left(I-K_{k} H_{k}\right) P_{k \mid k-1}
\end{gathered}
$$

where $x$ is the state vector, $u$ is the input vector, $z$ is the measurement vector, $P$ is the state covariance matrix, $Q$ is the assumed process noise covariance matrix, $R$ is the assumed measurement noise matrix, $I$ is an identity matrix, $K$ is the Kalman gain matrix, $f$ is the nonlinear prediction function, $h$ is the nonlinear observation function, $k$ is the discrete time index, and $A$ and $H$ are Jacobian matrices of the prediction and observation functions respectively with respect to the state vector. 


\section{Chapter 3 : Experimental Set-Up}

This section of the thesis describes the WVU research UAVs, the avionic systems implemented on the propulsion assisted control (PAC) test bed platform, the development of the avionic hardware, as well the flight testing operation conducted at WVU.

\subsection{WVU Propulsion Assisted Control Aircraft}

The WVU PAC aircraft was designed to be a versatile and reliable platform for the Flight Control Systems Laboratory (FCSL) research group. The PAC fleet is comprised of three aircraft, each with a particular research area and color: sensor fusion - Red, flight controls - Blue, thrust vectoring - Green. The aircraft were completely built in-house and instrumented by integrating off the shelf parts with custom printed circuit boards (PCB). The Blue aircraft below is the flight controls research aircraft shown with the original propulsion system implemented on the aircraft.

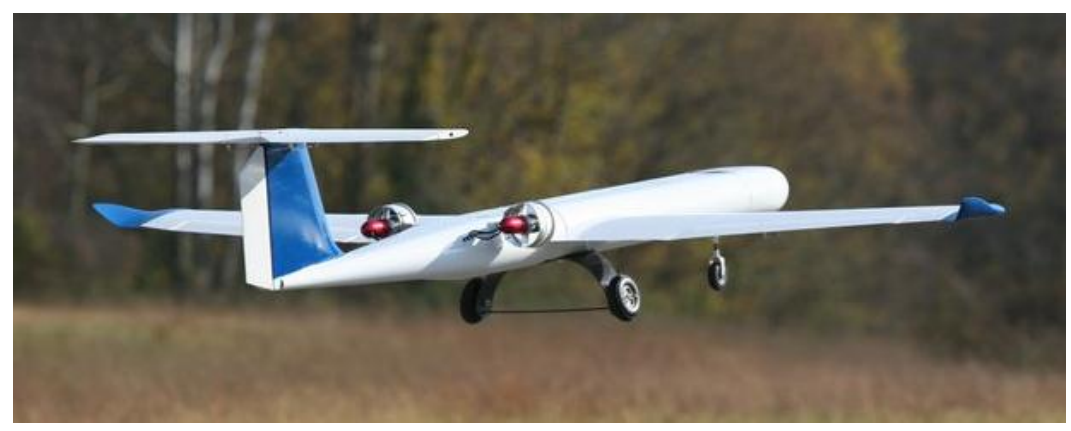

Figure 3.1: Blue PAC aircraft in flight

The aircraft are approximately $7 \mathrm{ft}$. in length, and with $7 \mathrm{ft}$. wingspans. Their takeoff weight is approximately $23 \mathrm{lbs}$. This includes approximately $4 \mathrm{lbs}$. of batteries and $4 \mathrm{lbs}$. of electronic payload. The aircraft have a T-tail configuration with winglets mounted on the tips of the wings. The construction of the PAC fleet was primarily conducted by fellow Master's student Zach Merceruio and Undergraduate researcher Marc Gramlich.

The fuselage is a composite structure made mainly of carbon fiber and fiberglass. There are wooden rails within the belly of the aircraft to allow for an avionics mounting point as well as additional structural rigidity. Bulkheads and formers are placed at interlocking locations to 
increase the rigidity of the fuselage. The aircraft has a small trough at the bottom center of the fuselage that runs almost the entire length, which is used for general wiring organization. This aircraft has conventional control surfaces: ailerons, elevators, and a rudder. The PAC fleet has been in operation since 2009.

The wing and horizontal tail are made of a foam core with plywood ribs and a carbon rod spar. This is covered in a thin layer of fiberglass and final layer of EconoKote $囚$. The fiberglass is for a general reinforcement of the wing and EconoKote provides a smooth surface. The nose cone of the aircraft is made of multiple layers of fiberglass and plywood bulkheads secured with carbon fiber ribbon. The nose cone is densely packed with various sensors as well as the front gear and steering servo. These sensors include the following: static and dynamic pressure, temperature, humidity, potentiometers for measurement of relative wind angles and a laser rangefinder for aircraft body to ground measurement. The sensors are discussed in detail in Section 3.2.5.

Throughout the development of the PAC fleet, alterations have been made to the propulsion system. Currently, the propulsion system consists of a pair of in-runner motors mounted in a 90 mm aluminum duct, each with a seven bladed glass filled nylon fan. These ducted fans have a maximum rotation speed of approximately $35 \mathrm{~K}$ revolutions per minute (RPM). The ducted fan motors have been dynamically balanced at low speeds by the vendor they were purchased from. This propulsion system provides a maximum combined static thrust of approximately $13 \mathrm{lbs}$.

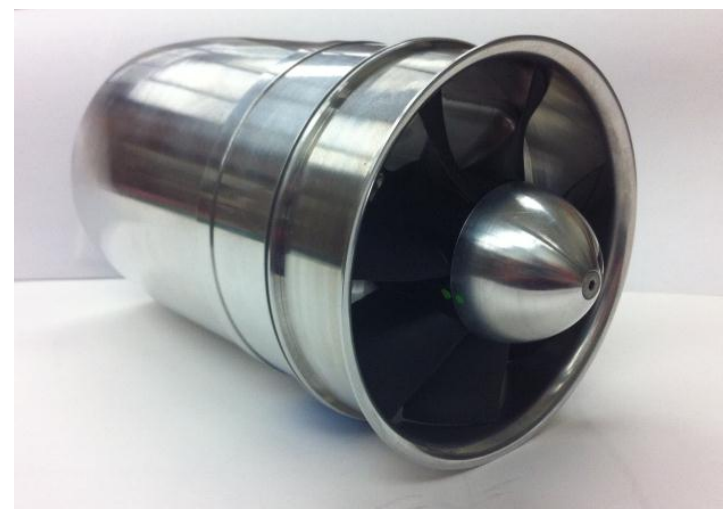

Figure 3.2: Current ducted fan in use on the PAC platform

The Blue and Green aircraft have the Gen-V avionics system onboard and the Red aircraft has a Quad-IMU data logging system. The major avionic designs throughout this graduate project 
revolved around the Gen-V system which is described in the next section, including details about the design and specifications of the system.

The locations of the major avionic components are label in Figure 3.3. The Green PAC aircraft was used throughout this graduate project for the collection of multiple GPS datasets.

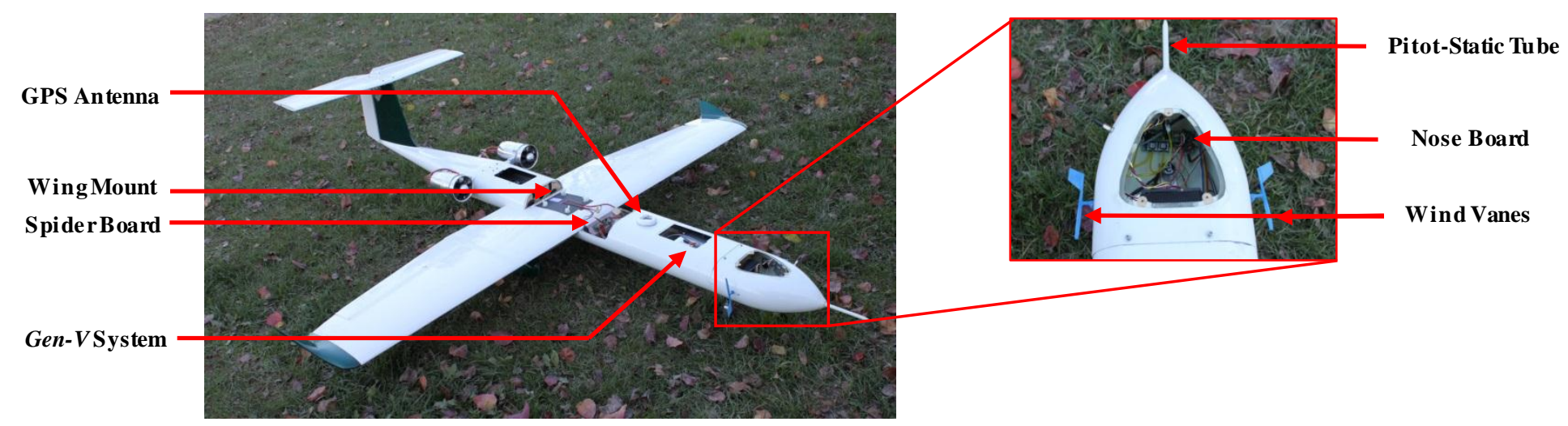

Figure 3.3: Locations of PAC aircraft avionics

\subsection{Propulsion Assisted Control Aircraft Avionics}

The main topic of this thesis is IGU product data used within an EKF for position estimation. However, advancements in the avionic hardware were a predominant part of this graduate project. The avionics onboard the PAC aircraft is a derivative of previous avionic system designs by fellow researchers [3], [4]. The development of the latest system, Gen-V, is described in detail by Jason Gross et al. [68]. Advancements of this system includes: design of the enclosure, hardware integration, reduction of assembly time, inclusion of more off-the-shelf parts, smaller form factor and less weight. The Gen-V system is described in detail within this chapter. 


\subsubsection{Gen-V Overview}

The Gen-V system consists a of custom peripheral connection PCB, a Diamond System's Athena II general purpose single board computer (SBC), a Diamond System's Jupiter MM-SIO DC/DC power supply, and a custom interface and signal distribution board. These electronic boards are on a PC/104 stack from top to bottom respectively. The SBC has an $800-\mathrm{MHz}$ central processing unit (CPU), 16-channels of 16-bit analog to digital conversion (A2D), and four serial ports. The power supply regulates the voltage for the SBC and also includes two serial ports. Figure 3.4 on the next page shows pictures of the front and rear of a Gen-V computer. The rear of the Gen-V system is densely filled with the following connections: main power input, auxiliary power out, GPS antenna connection, display, keyboard, commanded servo DB-9s, A2D DB-9, serial communication ports $2 \& 4$, indicating lights and switches.
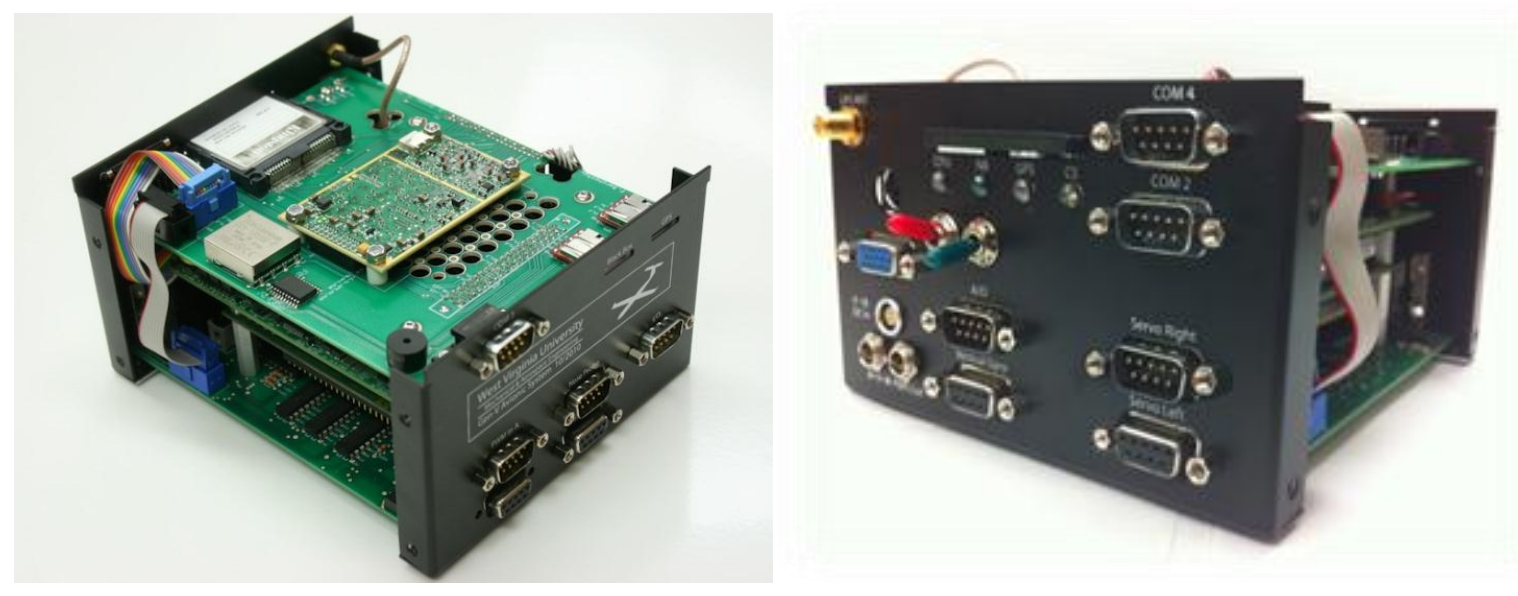

Figure 3.4: $G e n-V$ avionics stack in custom enclosure [68], [2] 


\subsubsection{Custom Enclosure}

The custom enclosure for the Gen-V system was designed in Solidworks® by the author. This was designed to be lightweight, informative to fellow researchers, and to decrease the electro-magnetic interference (EMI) to the electronic components outside of the system. This custom U-shaped enclosure was manufactured by Protocase ${ }^{\circledR}$ but was modified to decrease weight of the assembled Gen-V avionic system. Custom silk screening was also placed on the outside of this enclosure; the follow figure is a representation of what was produced.

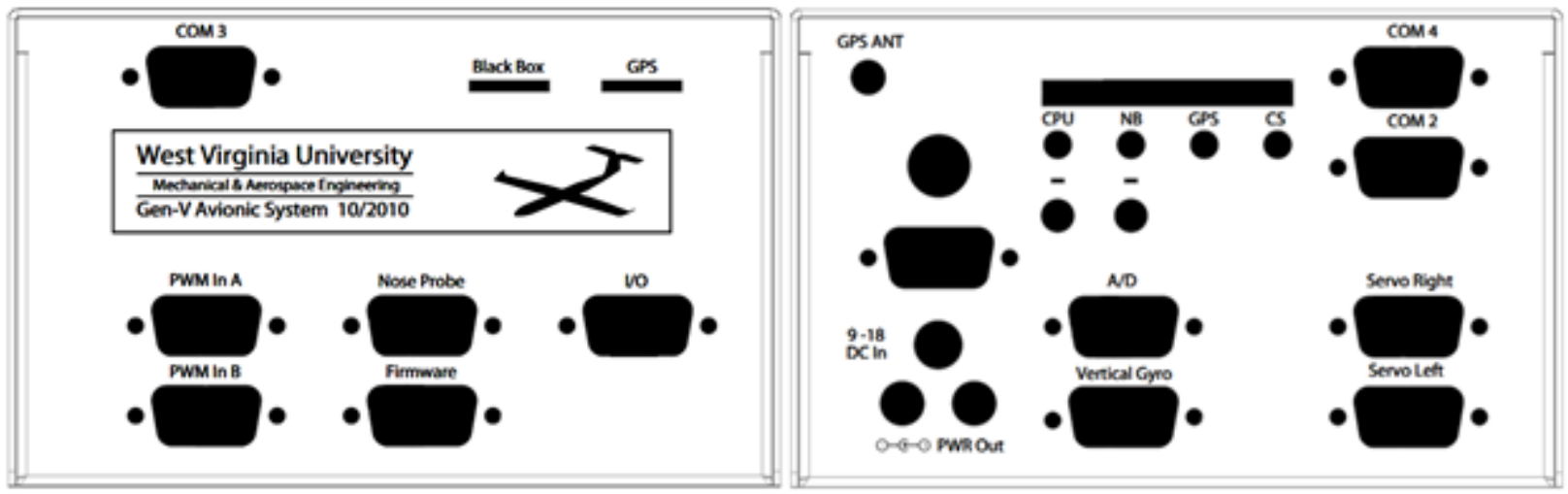

Figure 3.5: Silk screen design placed on Gen-V enclosure 


\subsubsection{Peripheral Connection Board of Gen-V}

The top PCB, or the custom peripheral connection PCB, was developed by the author. A design requirement of this top PCB was to decrease cabling, which included integrating NovAtel's OEMV®-1 GPS receiver into the Gen-V enclosure. This OEMV-1 receiver is reconfigurable, which enables the FCSL to choose particular information to be sent out. The most commonly used data log in this research group is NovAtel's BESTXYZ packet. This 112byte long message includes position $(x, y, z)$, velocity $(V x, V y, V z)$, the standard deviations of each of these measurements $\left(\sigma_{x}, \sigma_{y}, \sigma_{z}, \sigma_{V_{x}}, \sigma_{V_{y}}, \sigma_{V_{z}}\right)$, the number of viewed satellites $\left(N_{\text {view }}\right)$, the number of satellites used in the solution estimate $\left(N_{\text {sol }}\right)$, as well as other useful measurement information. All quantities are referenced to the WGS84 ellipsoid [69]. Message packets from this OEMV-1 can be sent out at a rate of $50 \mathrm{~Hz}$. The OEMV-1 receiver is compact with an option of three serial communication ports.

This graduate project was a contributing factor for including the option of additional serial ports connections. Particular NovAtel packets needed for this thesis were SATXYZ, and TIME. These data logs give information about the estimated satellite positions and the GPS receiver's clock respectively. The Green PAC aircraft was selected to have the option of recording the three packets discussed previously. The following figures below reveal the size and connecting points of the receiver to the top PCB.

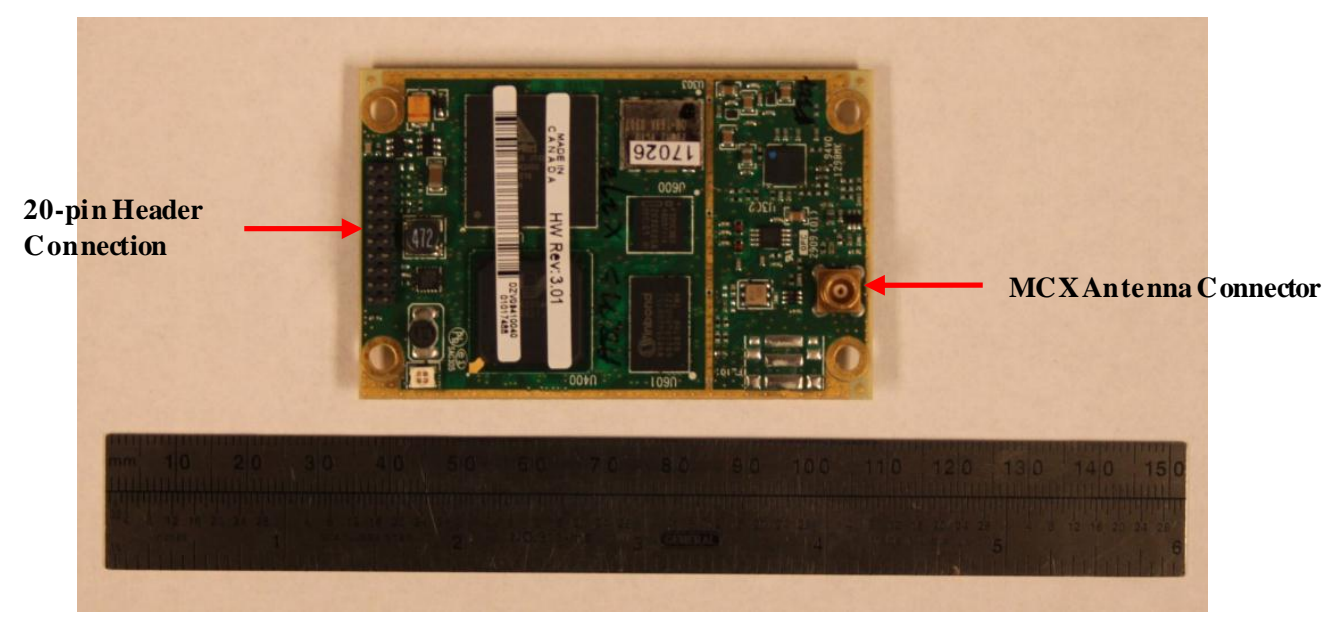

Figure 3.6: NovAtel's OEMV-1 GPS receiver 
The flight computer design before the top PCB included cabling for each DB-9 receptacle, keyboard, display, and compact flash connection. This increased production time, and raised concerns of reliability of the computer. The top PCB eliminated all previous cabling, excluding the display receptacle. Figure 3.7 shows a picture of the latest version of the top PCB and has labels for all major components.

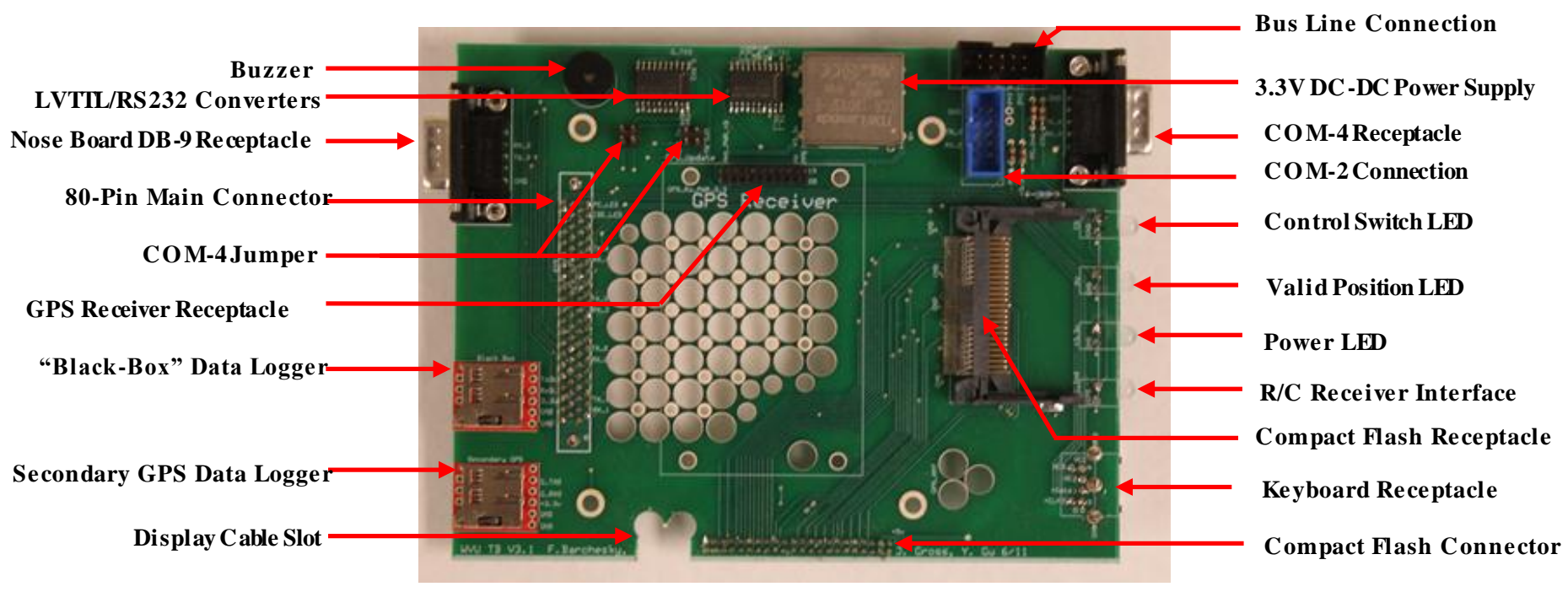

Figure 3.7: Custom peripheral connection board

Figure 3.8 shows a block diagram of the custom peripheral connection. The power for the top PCB is directly from the Gen-V power source, a $3 \mathrm{~S}$ LiPo battery. The red arrow lines indicate power traces and black arrow lines are signal traces. The top PCB includes indicator light emitting diodes (LED) (colored in light orange), a buzzer (colored in light green). All status lights and the buzzer are from the bottom PCB of the stack and are connected through a bus. All receptacles are colored in gray these include DB-9 connections, and a compact flash (CF) connection. This PCB includes a set of jumpers, dark black arrow lines, to allow for an auxiliary serial connection to the outside of the enclosure or to connect an additional serial port of the OEMV-1 GPS receiver to the SBC. The two serial connections between the OEMV-1 and SBC increases the amount of information that could be used in real-time. The green areas are the direct connections between the SBC and the top PCB. The larger of these two areas is an 80-pin connector and the smaller is specific to the CF connection. There are two small data loggers which record serial communication in real-time. This allowing the aircraft to have a "Black- 
Box", which records the downlink packet transmissions, and also a secondary data logger that interfaces with the GPS receiver.

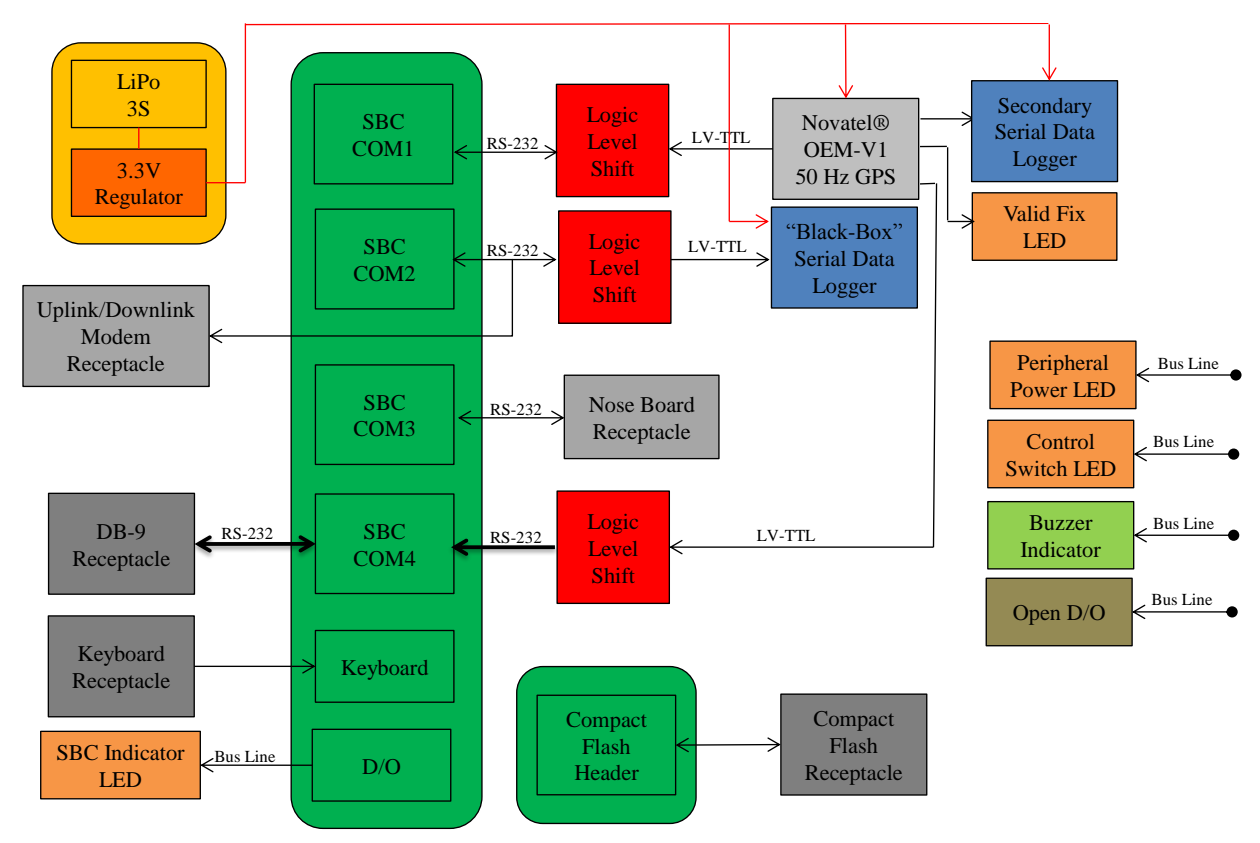

Figure 3.8: Custom peripheral connection block diagram

\subsubsection{Signal Distribution Board of Gen-V}

The custom interface and signal distribution PCB was design by fellow Graduate student Jason Gross. This PCB includes a Freescale ColdFire MOD-5213 ${ }^{\circledR}$ 66-MHz microprocessor which communicates between sensors and the SBC. The MOD-5213 is suited well for any deterministic application with its real time operating system and Netburner's integrated development environment [70]. The bottom PCB's MOD-5213 performs the following tasks: communication between with the Analog Device's digital micro-electro-mechanical system (MEMS) ADIS-16405 inertial measurement unit (IMU), reading the pilot commanded pulse position modulation signal (PPM), writing the pulse width modulating (PWM) servo commands that are derived from the commands of the SBC, collection of a couple of 12-bit A2D channels, and general DIO for some of the indication LEDs.

The bottom PCB has gone through an evolution of designs, which includes the incorporation of the top PCB. The bottom PCB allows the FCSL to easily configure pilot-in-the-loop or 
partially autonomous to fully autonomous aircraft modes. The signal distribution aspect of this PCB is architecturally designed such that in the event of a failure, the safety pilot will remain in control of the PAC aircraft.

The Gen-V system has the capability of producing nine unique PWM signal commands. These are usually connected to the following surfaces: left/right elevators, left/right ailerons, rudder, (2) throttle channels, and (2) thrust vectoring channels. This allows for independent control of any surface. The computer has inputs to 11 PWM channels, which are the same as the outputs with the addition of a "Kill-Switch" and a "Mode Switch". Due to the possibility of radio control interference propagated by the Gen-V system, the "Kill-Switch" was integrated to allow the safety pilot on the ground to turn off the SBC.

The "Mode Switch" is what dictates if the aircraft is in autonomous or manual pilot mode. There is a double-layer logic for fully autonomous control to be activated, and is defaulted to manual control. This double layer logic is described by Figure 3.9.

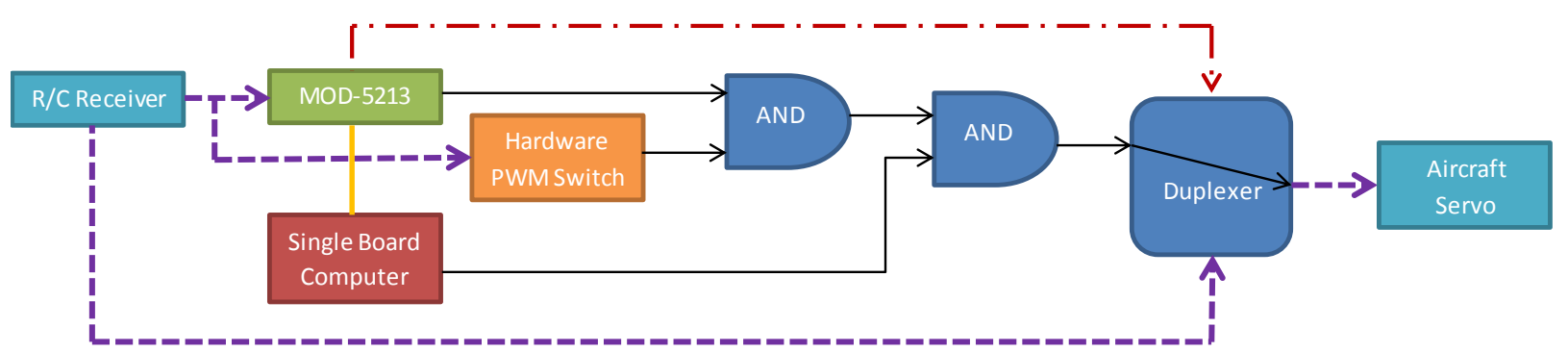

Safety Pilot PWM Commands

Control Laws Derived PWM Commands

Digital Output

Serial Communication

\section{Figure 3.9: Control mode activation block diagram}

The "Mode Switch" PWM signal is split to the MOD-5213 and a hardware PWM switch, then these are fed into an AND gate. This AND gate is fed to another AND gate which is also connected to the SBC. The output of the final AND gate is connected to the duplexer, which switches between the manual pilot commands and the control commands produced from the 
SBC. If any of the AND gates do not return a true result the duplexers will default to manual pilot control.

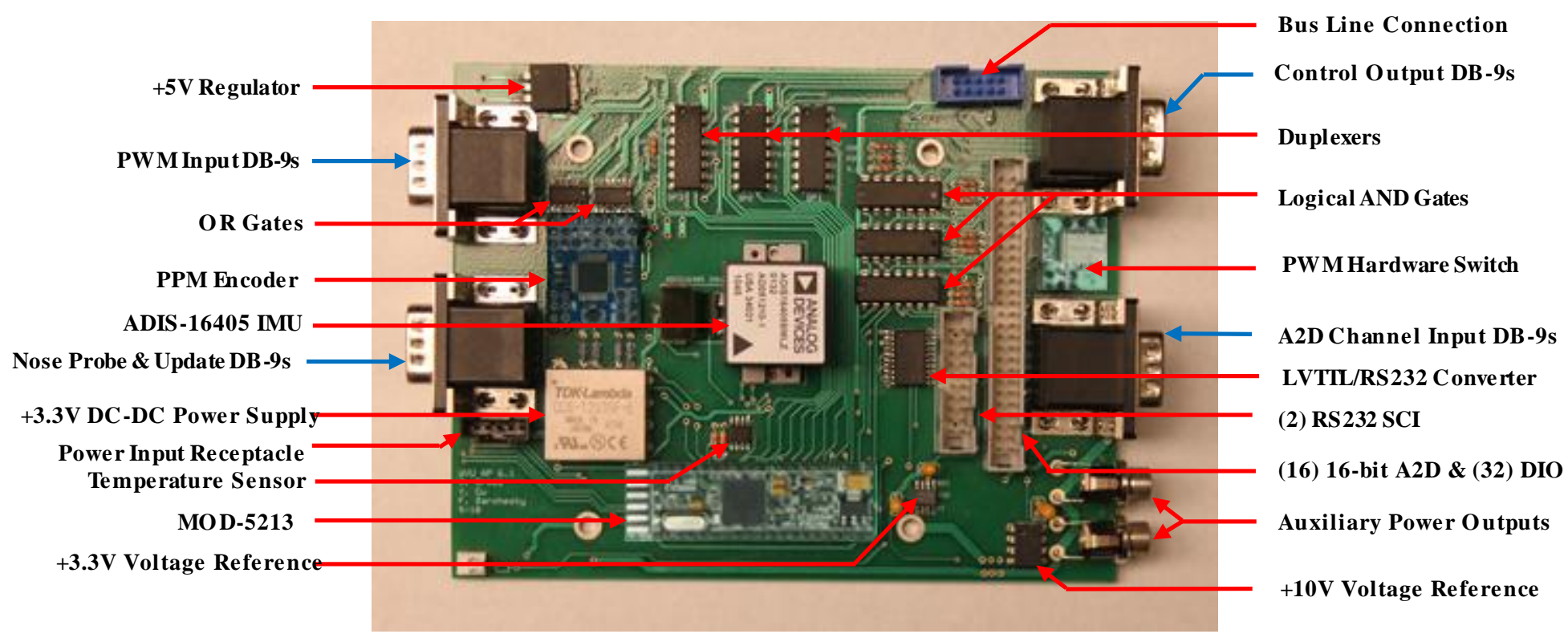

Figure 3.10: Bottom PCB fully assembled

Each of the components labeled in Figure 3.10 have an important role of the Gen-V system. The Bus-Line connects between the bottom PCB and the top PCB. This contains indicators as well as main power. The control output DB-9s are the nine PWM channels that are routed to the servos through a distribution board. The logical AND gates are the secondary layer of AND gates discussed above. The PWM hardware switch is an isolated hardware switch that determines to what setting the "Mode Switch" has been flipped. The A2D input DB-9s are also connected to the servo distribution board, and allow easy access and expandability to sensors onboard the aircraft. The LVTTL-RS232 converter allows the MOD-5213 to communicate with the serial ports of the power supply. The connection of power supplies serial ports to the bottom board are labeled next. The connection of the SBC's 16 channels of A2D conversion and 32 DIO to the bottom board is labeled thereafter. The last two components labeled on the right side of the figure are the auxiliary power outputs and $\mathrm{a}+10 \mathrm{~V}$ voltage reference for a consistent voltage for the A2D conversion of the SBC. 
Going from the top left side of Figure 3.10, the first labeled component takes the power of the $\mathrm{R} / \mathrm{C}$ receiver and regulates it to $+5 \mathrm{~V}$ for the following components to be powered: PPM encoder, duplexers, and OR gates. The PWM input DB-9s are labeled next, which are the main manual control commands that are sent from the R/C receiver. The PWM inputs are then fed into the OR gates to allow a layer of voltage protection for other components, which are labeled next. The PWM logical-high voltage of some modern $\mathrm{R} / \mathrm{C}$ receivers is $+6.6 \mathrm{~V}$ which can cause damage to sensitive components these OR gates outputs have a logical-high output voltage of $+5 \mathrm{~V}$. The PPM encoder, labeled after the OR gates, takes the outputs of the OR gates and converts them in a single PPM signal. The ADIS-16405 IMU is labeled next; this device provides 12-bit measurements of accelerations and angular rates with a range of \pm 10 Gs and $\pm 150 \% \mathrm{sec}$ respectively.

The nose probe DB-9 includes the R/C receiver power, the control command of the $9^{\text {th }} \mathrm{PWM}$ channel, power from the SBC power supply $(+12 \mathrm{~V}$ and $+5 \mathrm{~V}$ lines), four 16-bit $\mathrm{A} 2 \mathrm{D}$ channels and the necessary ground. The $+3.3 \mathrm{~V}$ DC-DC power supply is a switching voltage converter that powers the MOD-5213 as well as many other electronic on the bottom PCB. The power input receptacle is the connection point between the power supply and bottom PCB. The temperature sensor is a signal conditioned AD22103®, which provides an effective internal enclosure temperature. The next labeled component is the MOD-5213, whose duties have been previously explained. The final component is a $+3.3 \mathrm{~V}$ voltage reference which is used with the $\mathrm{A} 2 \mathrm{D}$ channels of the MOD-5213. 


\subsubsection{Nose Avionics Board}

An important component of the avionics system is the nose board. There are two versions of this PCB: one design that works with the Gen-V system and another that can connect with a different data logging system. The nose board has a MOD-5213, which is the mediator between the nose sensors and the Gen-V system. There were two major criteria of the design of the nose avionics board: small form factor, and reliable connections between it and the Gen-V system. The size of this printed circuit board is approximately 3 " $\times 1.5$ ". This exceeded expectations in its size, and makes the PCB a very compact easily mounted electronic package. The specifications of the sensors connected to the nose are as follows:

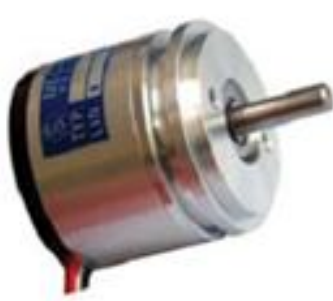

Figure 3.11: MP1545AS

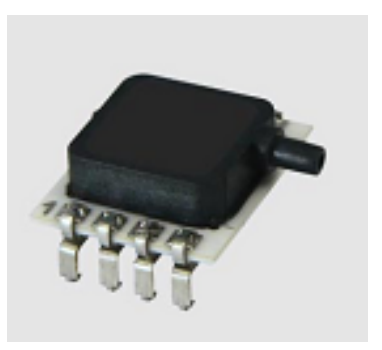

Figure 3.12: HCE0811ARH8P5
Relative wind angles $(\alpha, \beta)$ are measured by vanes attached to a MP-1545AS single-turn inductive contactless potentiometer. On the PAC platform there are two measurements of the angle of attack, $\alpha$, and one measurement $\mathrm{f}$ or side slip, $\beta$. These potentiometers are produced by P3 America ${ }^{\circledR}$ and have 100 degrees of measurement angle with a linearity of $\pm 0.5 \%$ [71]. The potentiometers are directly connected to the Gen-V 's A2D channels for higher reliability and resolution of measurement.

Dynamic and static pressures $\left(P_{d}, P_{s}\right)$ are measured by a set of Sensor Technics miniature amplified pressure sensors, HCEM020DUE8P3 and HCE0811ARH8P3 respectively. These digital sensors communicate through the serial peripheral interface (SPI) bus. They have a 14-bit resolution, and are calibrated and temperature compensated by the manufacturer [72]. Availability of sensors dictated the use of a $+5 \mathrm{~V}$ version which required integrating a logic level translator on the PCB. 
Outside air temperature and relative humidity $(R H)$ are measured

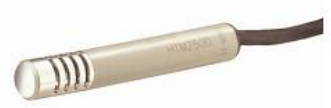

Figure 3.13: HTM2500LF

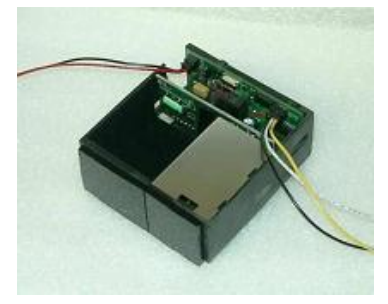

Figure 3.14: RS400 by Measurement Specialties® HTM2500LF transducer. This has a negative temperature coefficient (NTC) thermistor with a fast response time of 10 seconds and the calibrated humidity sensor with a response time of five seconds. This transducer was selected due its high reliability and long term stability especially with respect to the humidity sensor [73].

An electronic laser range finder is used for range measurements between the aircraft body and ground. This sensor, RS400, is produced by Opti-logic $®$ with a range of approximately 365 meters with a resolution of 0.2 meters [74]. The RS400 communicates with the MOD-5213 through RS232 serial protocol.

The nose PCB is connected to the Gen-V system in two locations: the COM-3 and nose probe DB-9s. These connections are fixed and soldered directly to the PCB to decrease the amount of strain on the wires. Figure 3.15was produced to illustrate how these sensors are connected and powered by the nose PCB. Color conventions are the same as Figure 3.8.

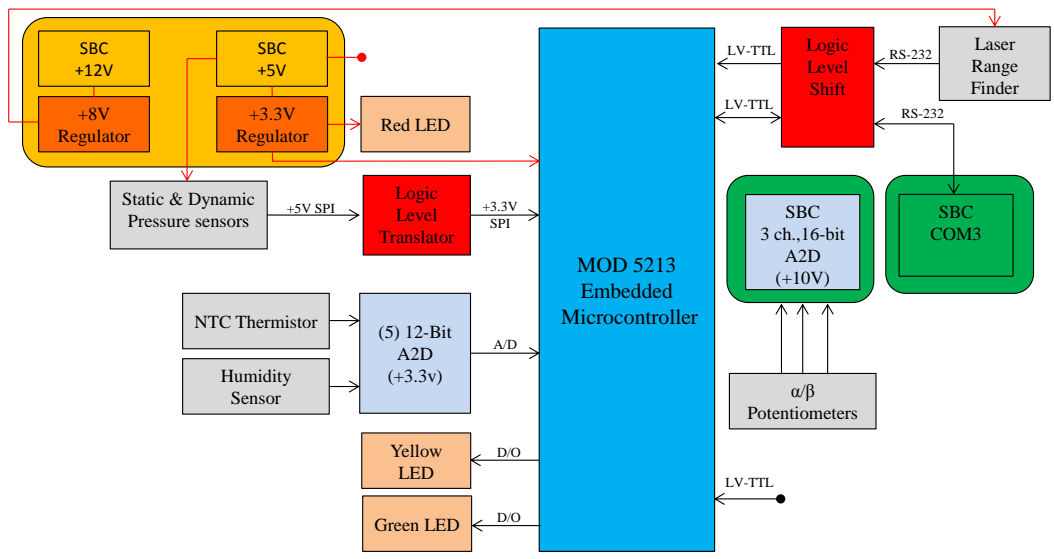

Figure 3.15: Nose PCB block diagram

The nose board has three indication lights: one for $+3.3 \mathrm{~V}$ power and two connected to digital outputs. These have been programmed to notify a user that the pressure sensors and laser range 
finder are communicating with the MOD-5213. Figure 3.16 is a picture of a nose board for the Blue/Green aircraft. There is a similar design for the Red aircraft however it demanded numerous alterations due to different communication architecture and power available. There are four major components underneath the MOD-5213 that were not labeled: logic level translator for communication to the pressure sensors, LVTTL-RS232 level shifter for communication between the rangefinder and MOD-5213, a $+3.3 \mathrm{~V}$ voltage reference for precise A2D conversion, and a NOT gate that enables a layer of protection for the general purpose timers (GPT).

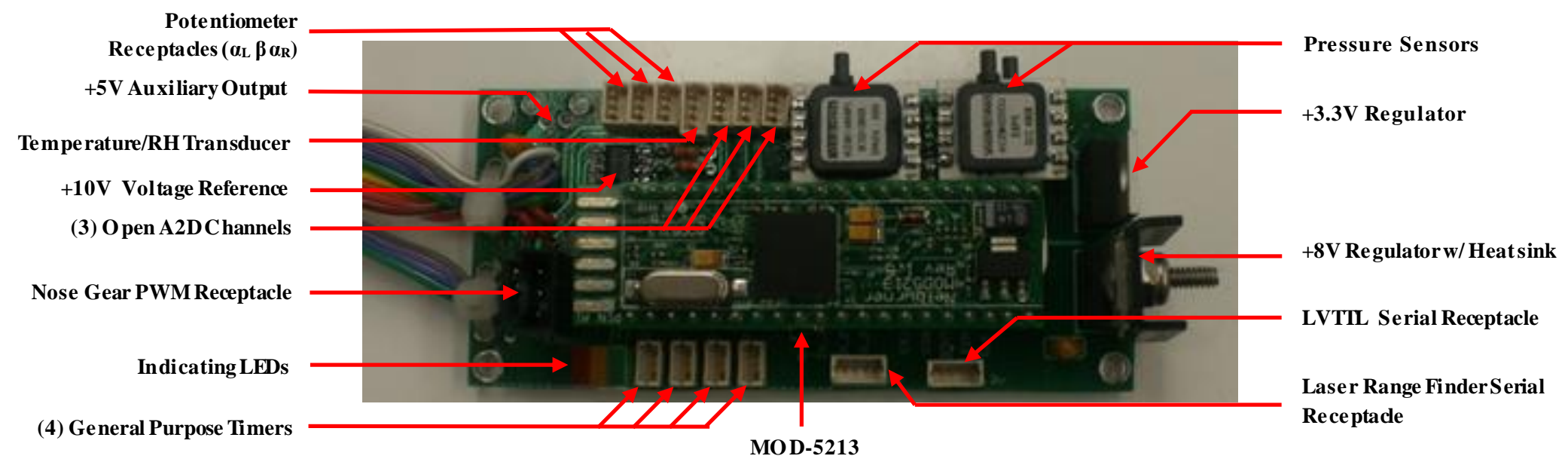

Figure 3.16: Fully assembled Blue/Green PAC aircraft nose PCB

\subsubsection{External Signal Distribution Board}

Another important component of the Gen-V system is an external signal distribution board, which will be referred to as the spider board. This PCB is called the spider board due to the many wire connections soldered directly to it. The spider board serves mainly as a control command PWM distribution board; this is a permanent fixture of the aircraft for organized wires to the servo motors. Due to the design of the PAC aircraft, the wing is removable, which requires the aileron servo channels to be (dis)connected easily. The spider board allows a reliable and accessible connection point for the servos. This PCB also allows access to the Gen-V system's external A2D channels. 


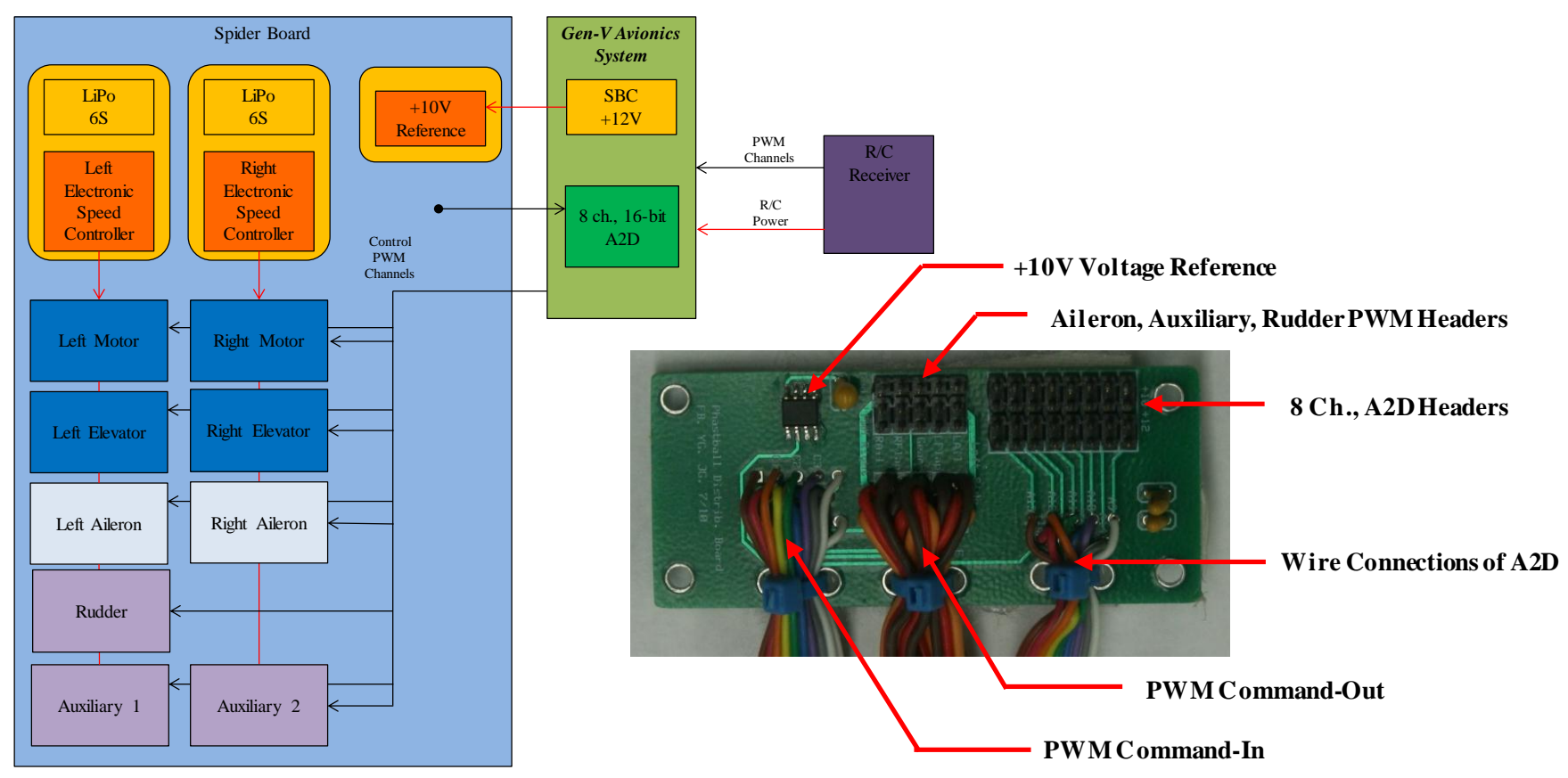

Figure 3.17: Spider board block diagram and fully assembled picture

Within Figure 3.17, the power distribution of the spider board incorporates a layer of safety. The power of each ducted fan $(6 \mathrm{~S}-\mathrm{LiPo})$ is converted by the electronic speed controllers (ESC) to be used for the servos. On the spider board the power of the left and right control servos are connected to their respective ESC. If in flight one of these batteries would fail or become disconnected, only that respective side of the aircraft's control surfaces would become partially floating. "Partially" is included in the previous statement because the rotation resistance within the servo will not allow the surface to move easily. The other half of the surfaces should provide enough maneuverability to land safely. 


\subsection{WVU Flight Testing}

The flight testing operation at WVU is conducted at the Jackson's Mill flight testing facility located in Jane Lew, West Virginia. The Jackson's Mill facility is a privately owned airfield of WVU, the name of the airfield is called Louis Bennett Field with FAA identifier-WV23. This airfield has an asphalt runway with a length of $3195 \mathrm{ft}$. For more information specifically about the airfield a brief description can be read in [75].

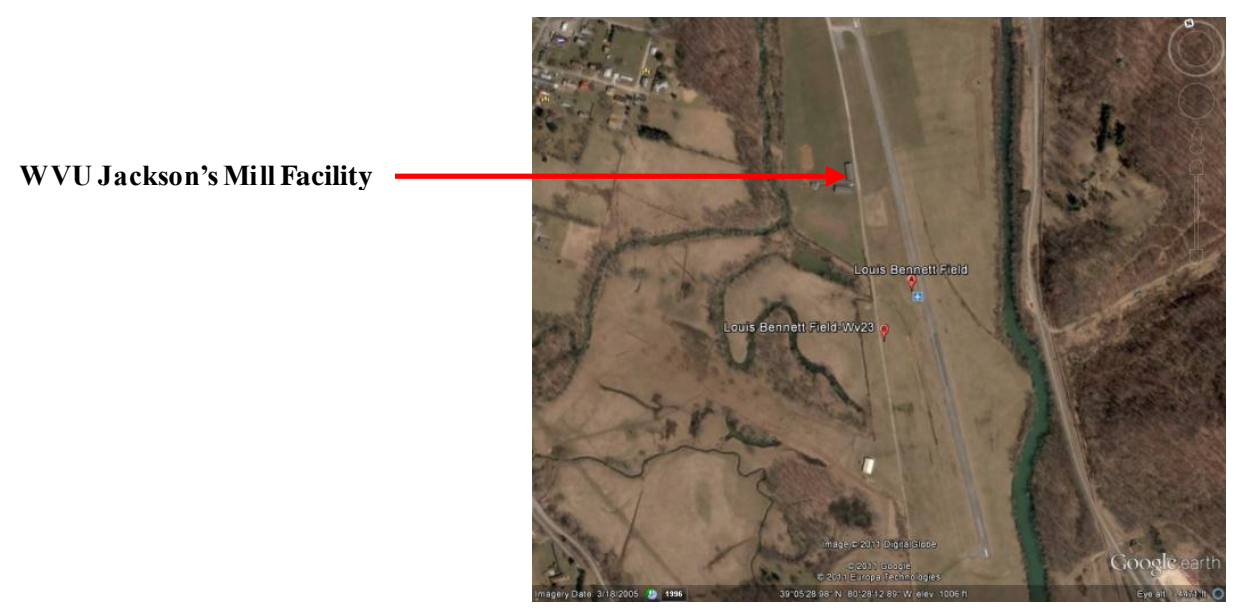

Figure 3.18: Louis Bennet Field

Many research topics within the FCSL, including this graduate project, use the flight testing data that is obtained at this facility. The research aircraft are transported to and from this facility in a box truck that is outfitted with a ground station and other necessary equipment. This ground control station currently is in its developmental stages. For the purpose of UAV research, this station has the ability of up and downlink of information to the PAC aircraft, recording of wind speed, wind direction, temperature, humidity, and the GPS location. 


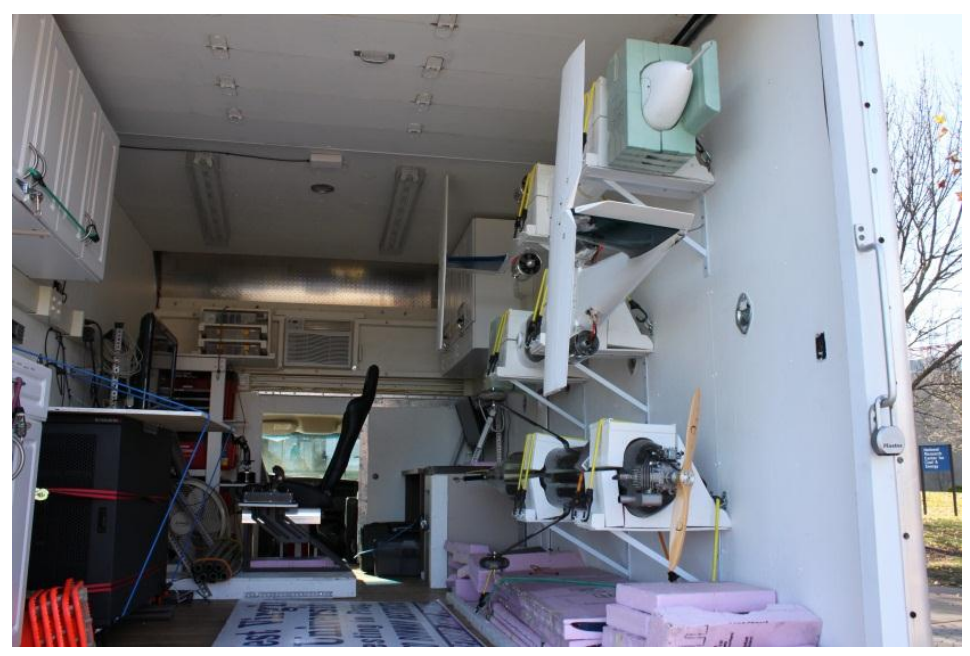

Figure 3.19: Transportation of aircraft

Since the flight testing at WVU is weather dependent, most flights occur between March and November. All of the members of the FCSL, which includes undergraduate students, graduate students, post-doctoral fellows, and faculty advisors, participate in the flight testing operation. The flight data used for this study was recorded in the 2011 flight season. 


\section{Chapter 4 : Development of MATLAB Tools}

\subsection{Overview of MATLAB Tools}

The MATLAB tools developed allows the importation of most IGS products and data types. These include precise ephemerides, clock solutions, ionospheric grids and raw daily broadcast compilations. The particular formats of these different sets of information and how they have been used are discussed in the next section. Also within Section 4.1.2 other useful functions created in conjunction with these MATLAB tools will be discussed and presented. The IGS information expansions include estimating a GPS satellite's velocity through the use of a conventional method, and interpolation strategies to provide a precise satellite position for the use in a real-time and/or near real-time application, which are discussed in Sections 4.2 and Section 4.3 respectively.

\subsubsection{IGS Products and Data Formats}

IGS has a variety of products and raw data. Each different grade or type of IGS precise satellite orbits and clocks are in the Standard Product 3 format (SP3). A brief history about this file format is discussed before going into detail about the extended SP3-c file format. The NGS standard GPS orbit format SP1 was introduced in 1985 [76]. Shortly after this, the necessity to modify the format was apparent. Slight modifications of this format were added without altering the existing software or general structure of the format. The NGS format did not however include satellite clock corrections. This was due to the assumption that geodetic applications would be in differential mode. The SP1 format is standard and not limited to just geodetic applications, therefore to serve a wider community, Remondi in 1989 proposed a new NGS orbit format [77]. This was the original SP3 format (SP3-a) it was then modified and adopted in 1991 [78]. In 1998, Gurtner and Rothacher's defined the combination of GPS and GLONASS orbits thus creating the SP3-b format. In 2000, modifications of the format were discussed at the IGS Analysis Center Workshop due to a desire for the inclusion of clock accuracy information. This resulted in another discussion in 2002 at the IGS Network, Data, and Analysis Center Workshop. From this discussion, more ideas about the following arose: flags for clock events, orbit maneuvers, clock predictions, orbit predictions, correlation information between satellite 
coordinates, and satellite clock correction. Slight modifications have been made since to include other time reference systems such as International Atomic Time (TAI).

Each SP3 file includes the following information: number of total satellites, satellite PRN numbers, satellite positions, coordinate frame in which the positions are referenced, satellite clock corrections, interval of satellite information, total number of individual satellite epochs, satellite orbit accuracy, analysis centers used in the formation of the product, GPS week and seconds of the week associated with the first epoch, etc. For more information about this format, see $[\mathbf{7 9 , 8 0}]$.

Figure 4.1 is a sample section of an IGS SP3-c extended format document. The accuracy code, AccCode, is related to the standard deviation of a particular satellite's position throughout the dataset. More information specifically about the accuracy and precision of the IGS data can be read in Griffiths and Ray's article [81]. The accuracy codes are still in place in the SP3-c file for backwards compatibility to SP3-a. The following equation can be used to determine the standard deviation of the satellites position from the accuracy code: $\sigma=2^{\text {AccCode }}$. In the SP3-c version the standard deviations of the position components and clock are exponents of a floating point base. This base number is usually $1.25 \mathrm{~mm}$ for position and $1.025 \mathrm{psec}$, as in Equation 4.1. This is an example calculation to determine the standard deviation of the $x$-position of satellite PRN09 in the sample SP3-c document.

$$
\begin{gathered}
\sigma_{x}=b^{N_{x}} \\
\sigma_{x}=(1.25)^{10}=9.3132 \mathrm{~mm}
\end{gathered}
$$

The importation of every different type of IGS satellite orbit product was written in MATLAB so that a complete understanding of each individual set of products would be known. This also enables analyses to be conducted in different ways due to the availability of all product types. 


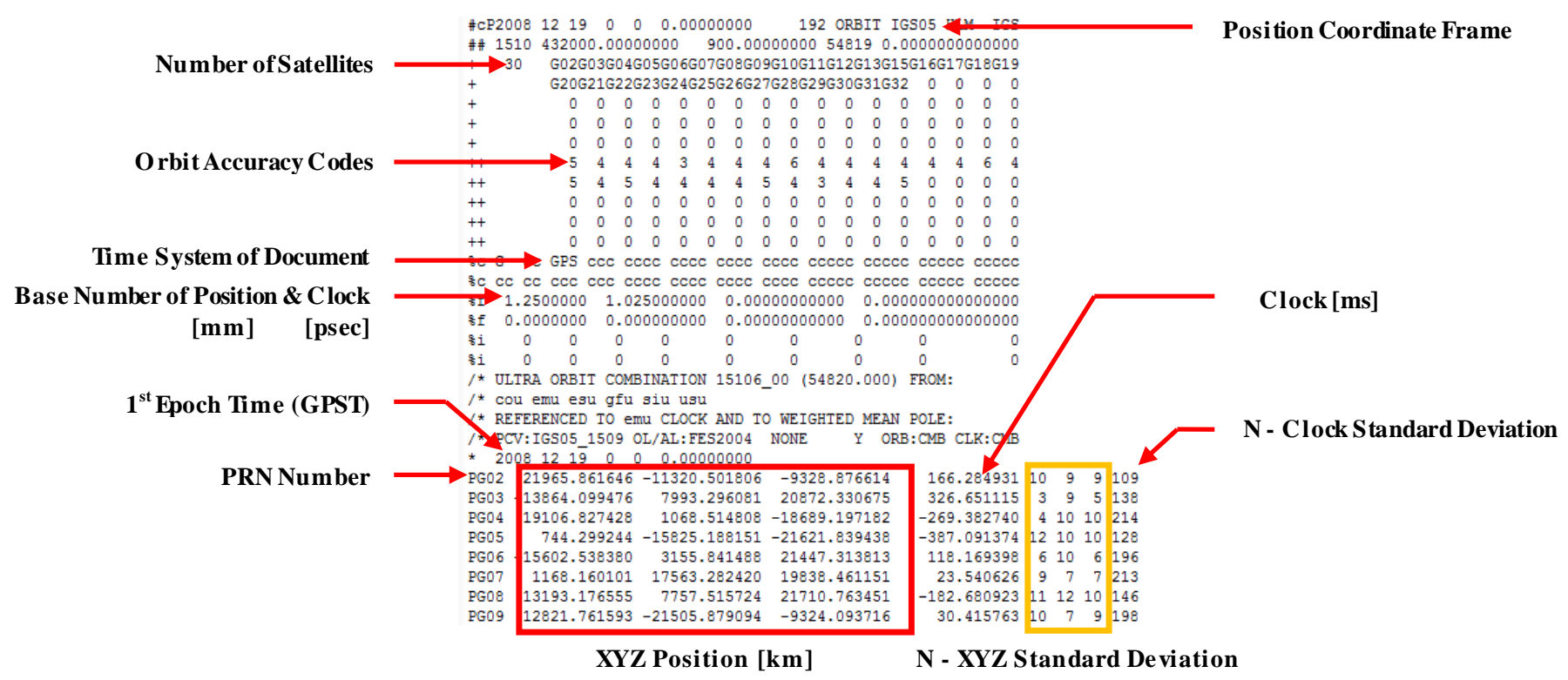

Figure 4.1: Sample of an IGU SP3-c file

For a more conventional approach, the use of the raw broadcast data was also pursued to be imported into MATLAB. Most IGS broadcast data is in the RINEX 2 format. Gurtner and Mader published the RINEX 2 format in 1990. A revision was made for a new treatment of antispoofing data, this is called RINEX 2.1 [82].

The RINEX 2 format includes the entire navigation message for each satellite throughout an entire day. The navigation message includes orbital parameters to calculate the satellite's position. For more information about the navigation message see Section 2.1.5, or for a detailed report see the following technical report [83]. Appendix B of this thesis contains a section of this report which describes the contents of the RINEX file. The broadcast raw data that was used in this graduate project was the compilation of all daily ephemerides from individual stations. These daily compilations are catalogued with respect to the day of the year (DOY). Figure 4.2 is a sample of a Broadcast RINEX file. 


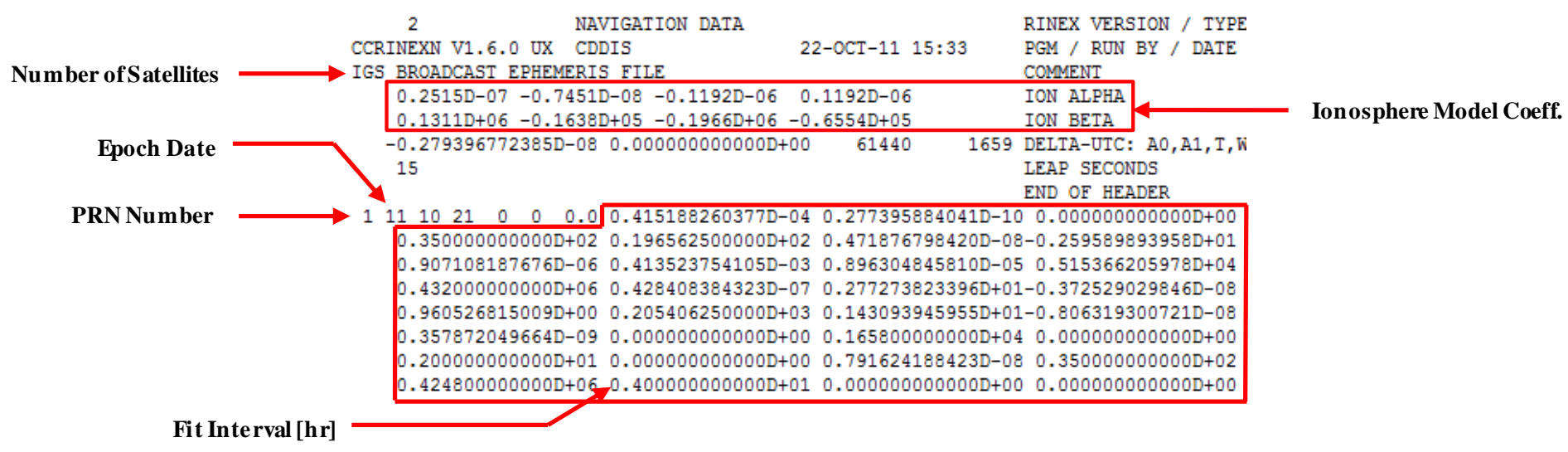

Figure 4.2: Sample of a RINEX file

In the RINEX file shown in Figure 4.2, the ionospheric model coefficients are in the first box, and the other enclosed group of text is the respective satellite's navigation message, which includes Keplerian orbital parameters as well as the space vehicle health. These broadcast parameters have a usual validity interval or fit interval of four hours, containing the two hours before and after their respective epoch. In the sample RINEX file the viewed satellite, PRN-1, has a fit interval of four hours. A brief analysis between broadcast data and precise IGS product data follows.

Initially in this project, the use of the IGS precise products was intended; however for realtime use the predicted IGU product only applicable. Figure 4.3 shows the difference between the $\mathrm{XYZ}$ components of the position of the satellite PRN-8 throughout the entire predicted portion of the IGU product. Since IGU products are in intervals of 15 minutes, the satellite's position was calculated with the broadcast orbital parameters with the most applicable fit interval. This causes the large changes in position error throughout the dataset. Overall the difference is larger than expected due to how IGS reports their error. The error reported in Table 2.1 is a one-dimensional mean root mean square (RMS) values over the three XYZ components. 


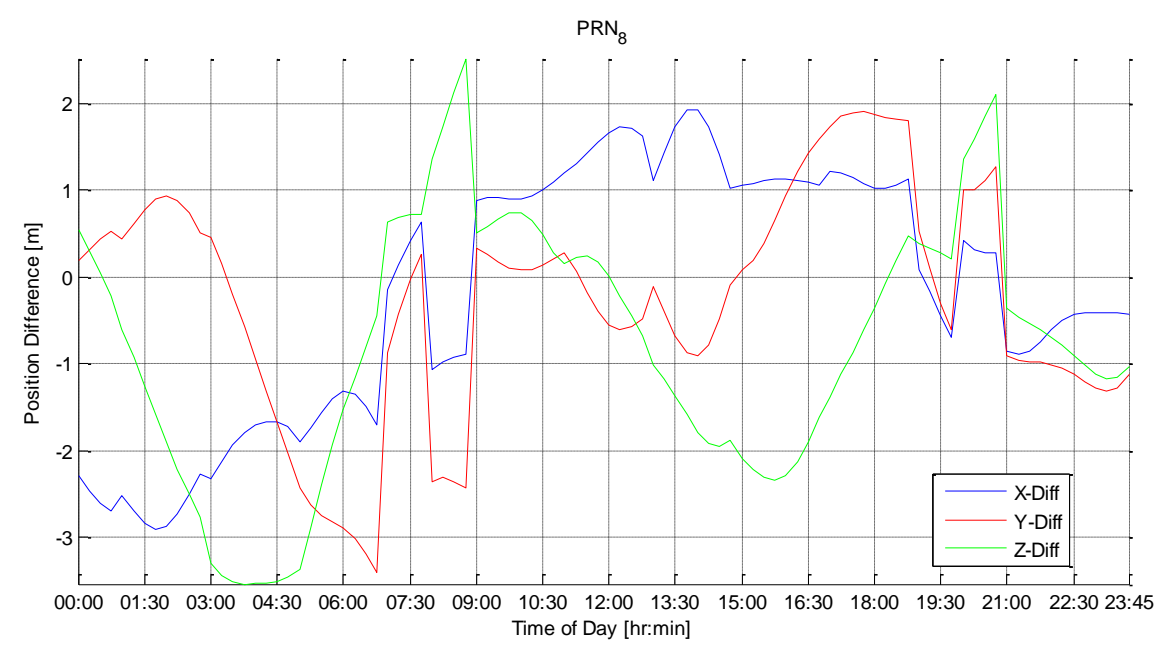

Figure 4.3: Position differences of broadcast data and IGU orbit

Figure 4.3 displays an optimistic approximation of the orbital position errors that a user's GPS receiver would be subjected to. This is an optimistic approximation because if a satellite's navigation is not updated for any reason before the fit interval ends the satellites position will drift. This phenomenon can be viewed within certain satellites in the IGS raw broadcast files, since there are missing satellite navigation messages. Figure 4.4 shows what would happen if a GPS receiver would not have an updated navigation message beyond the first fit interval. This means that the initial errors will be identical to Figure 4.3 until there is an updated navigation message. In Figure 4.4, the duration of time the satellite's position drifts is due to a missing navigation message within discussed broadcast file. 


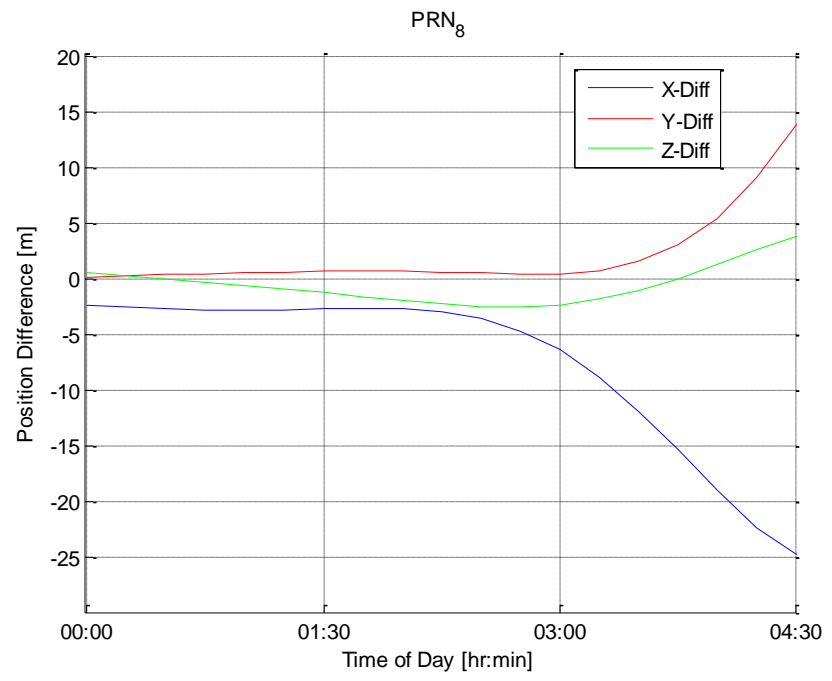

Figure 4.4: Position Error of PRN-8 without a navigation message update

The last file format that is discussed is the IONEX format. Through the use of a GNSS tracking network, it is possible to extract additional information about the Earth's atmosphere. IGS has a set of pilot programs that are estimating atmospheric parameters, including tropospheric zenith path delay, Ultra-Rapid tropospheric zenith path delay, Final ionospheric total electron content (TEC) grid, and Rapid ionospheric TEC grid. Table 4.1: IGS atmospheric product table Table 4.1 is provided to show their respective accuracy, latency, update frequency and sample interval. 
Table 4.1: IGS atmospheric product table [60]

\begin{tabular}{|l|l|l|l|l|}
\hline $\begin{array}{l}\text { Atmospheric } \\
\text { Parameters }\end{array}$ & Accuracy & Latency & Updates & $\begin{array}{l}\text { Sample } \\
\text { Interval }\end{array}$ \\
\hline $\begin{array}{l}\text { Final } \\
\text { tropospheric } \\
\text { zenith path } \\
\text { delay }\end{array}$ & $4 \mathrm{~mm}$ & $<4$ weeks & weekly & 2 hours \\
\hline $\begin{array}{l}\text { Ultra-Rapid } \\
\text { tropospheric } \\
\text { zenith path } \\
\text { delay }\end{array}$ & 6 mm & $2-3$ hours & 3 hours & 1 hour \\
\hline $\begin{array}{l}\text { Final } \\
\text { ionospheric } \\
\text { TEC grid }\end{array}$ & $2-8$ TECU & $\sim 11$ days & weekly & $\begin{array}{l}\text { (lon) } \mathrm{x} 2.5 \\
\text { deg } \\
\text { deg (lat) }\end{array}$ \\
\hline $\begin{array}{l}\text { Rapid } \\
\text { ionospheric } \\
\text { TEC grid }\end{array}$ & $2-9$ TECU & $<24$ hours & daily & $\begin{array}{l}\text { (lon) } x 2.5 \\
\text { deg (lat) } \\
2 \text { hours; } \\
\text { (leg }\end{array}$ \\
\hline
\end{tabular}

The IONEX files include a global map of the total electron content (TEC) of the ionosphere. The TEC maps have a grid size of $5^{\circ}$ longitude, and $2.5^{\circ}$ latitude. Within these files, the RMS error maps and single layer height are also included. These files have a similar header format as the RINEX file. A sample IONEX file is provided in Figure 4.5. 

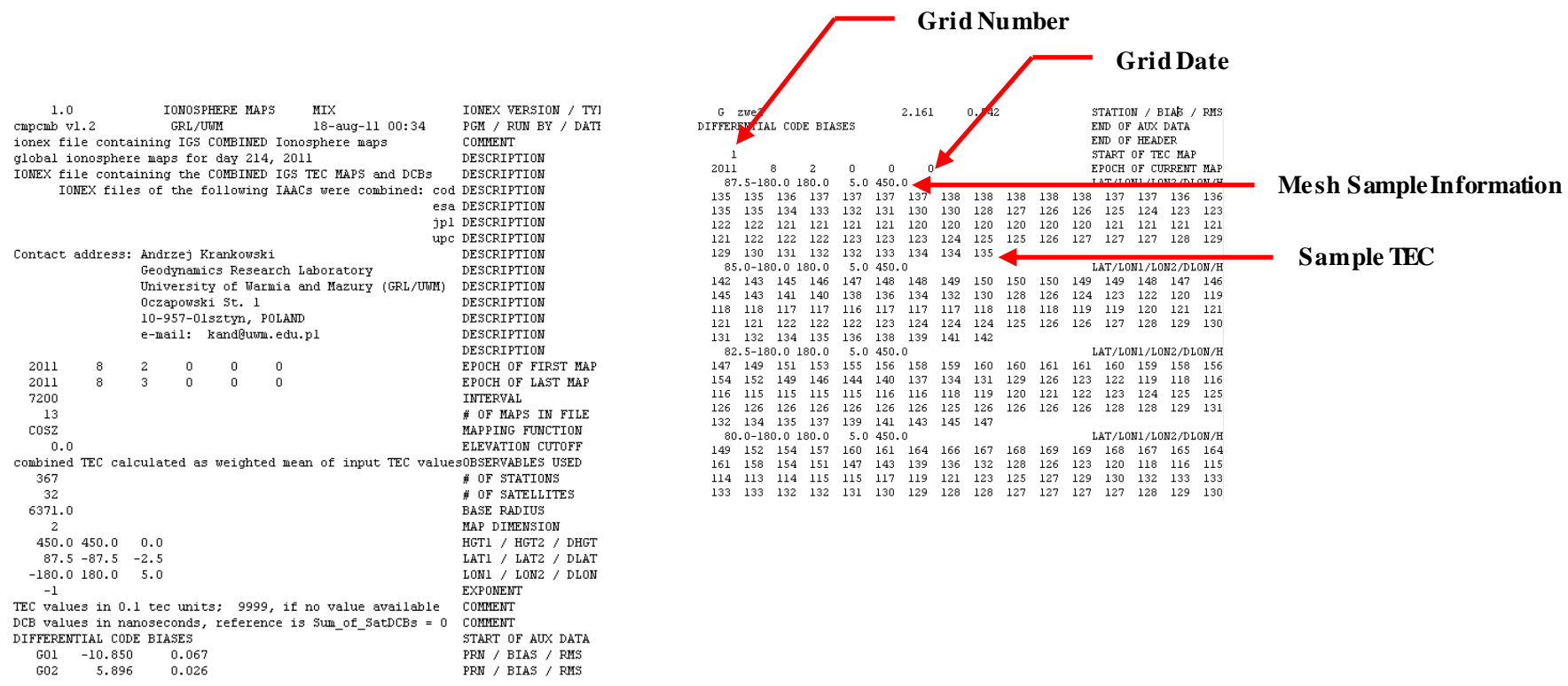

Figure 4.5: Sample IONEX file

Since these products are not predictable the use of this information in a real-time application is not possible. Due to this latency the ionospheric products were not selected to be used within comparison of this graduate project. However, a MATLAB tool was created and used to understand the variations of the TEC grid. As stated previously, the ionosphere becomes ionized by solar radiation which in turn results into a higher mean TEC near the equator of the Earth. The following set of figures was created from the developed MATLAB tool which shows how the TEC changes throughout a day. 

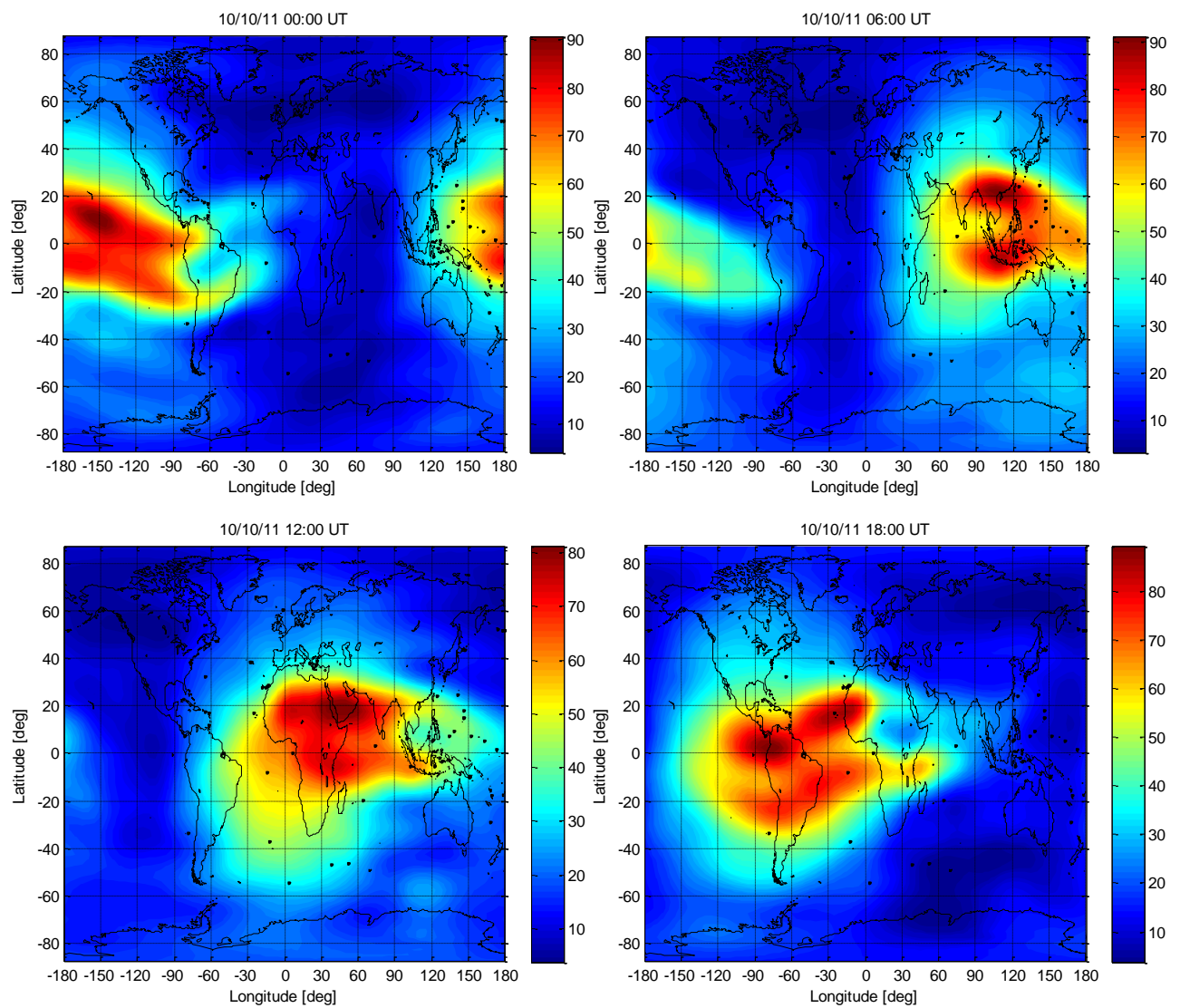

10/11/11 00:00 UT

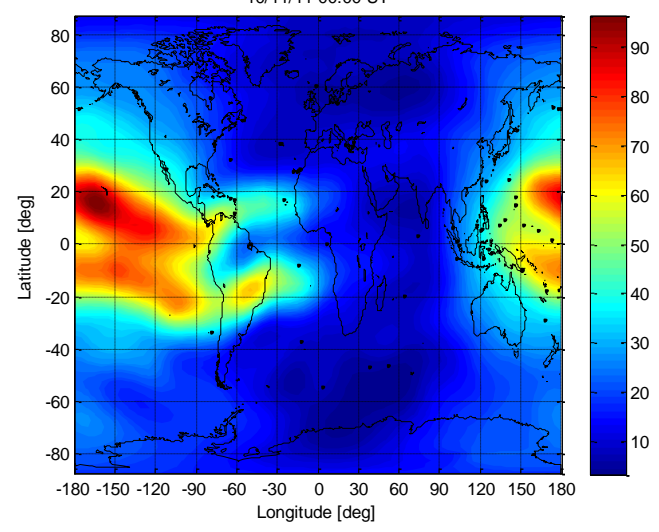

Figure 4.6: Total electron content change throughout a day

As the Earth rotates about its axis, the ionization causes larger values of free moving electrons. The variation between two days can be seen from the differences between the first and last subplots. An approximation of the time delay caused by the ionized electrons could be determined by: $\tau_{\text {ion }}=\operatorname{TEC}\left(40.3 / c f^{2}\right)$; where $c$ is the speed of light and $f$ is the GPS frequency. For proper modeling of these errors, the obliquity in which the GPS signal passes through the 
ionosphere should also be included. For more information about ionospheric errors see Section 5.3.2 of Misra and Enge's [42], or Section 5.2.2 of Grewal et al. [39], or Section 7.2.4.1 of Kaplan et al. [43].

\subsubsection{Developments from Created MATLAB Tools}

From these tools, clear and concise sets of data/products can be used within MATLAB. The advances of past research areas within this graduate project are first described. Thereafter the implications of the developed MATLAB tools are discussed. An extension of these tools was the development of a GPS/INS Sensor Fusion Simulation Environment [84]. This environment uses IGR product data for a particular day of interest as well as GPS recorded data to model satellite visibility. With the use of the recorded GPS satellites and the IGS satellite positions a comparison between observed and viewed satellites can be conducted. A brief description about this environment follows.

Portions of this environment have been configured to be used within other research areas. For example the viewed number of satellites by a GPS receiver. The viewed number of satellites was determined by an antenna viewing cone. The following figure, taken from the original publication, is included to understand the concept of this viewing cone.
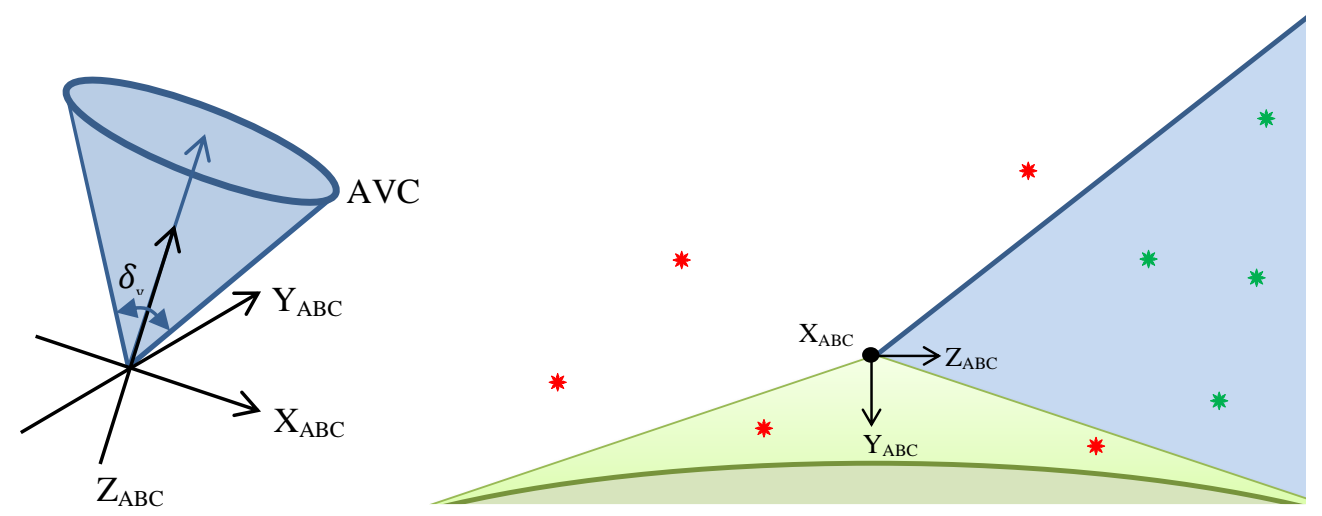

Figure 4.7: Antenna viewing cone and Earth's shadow [84]

The viewing angle of the antenna, $\delta_{v}$, is easily tuned for a particular GPS receiver. Based upon the earth's shadow as well as the orientation of the aircraft, the viewed number of satellites can be obtained. Within the original document, the number of satellites used within the GPS receiver's position/velocity estimation was directly correlated to the number of solution used 
number of satellites. Alternative methods based upon the geometric properties of the viewed number of satellites are easily adaptable.

Another tool that was created allows a play-back of a GPS receiver's collected data. The following figure shows the overall layout of the SIMULINK model.

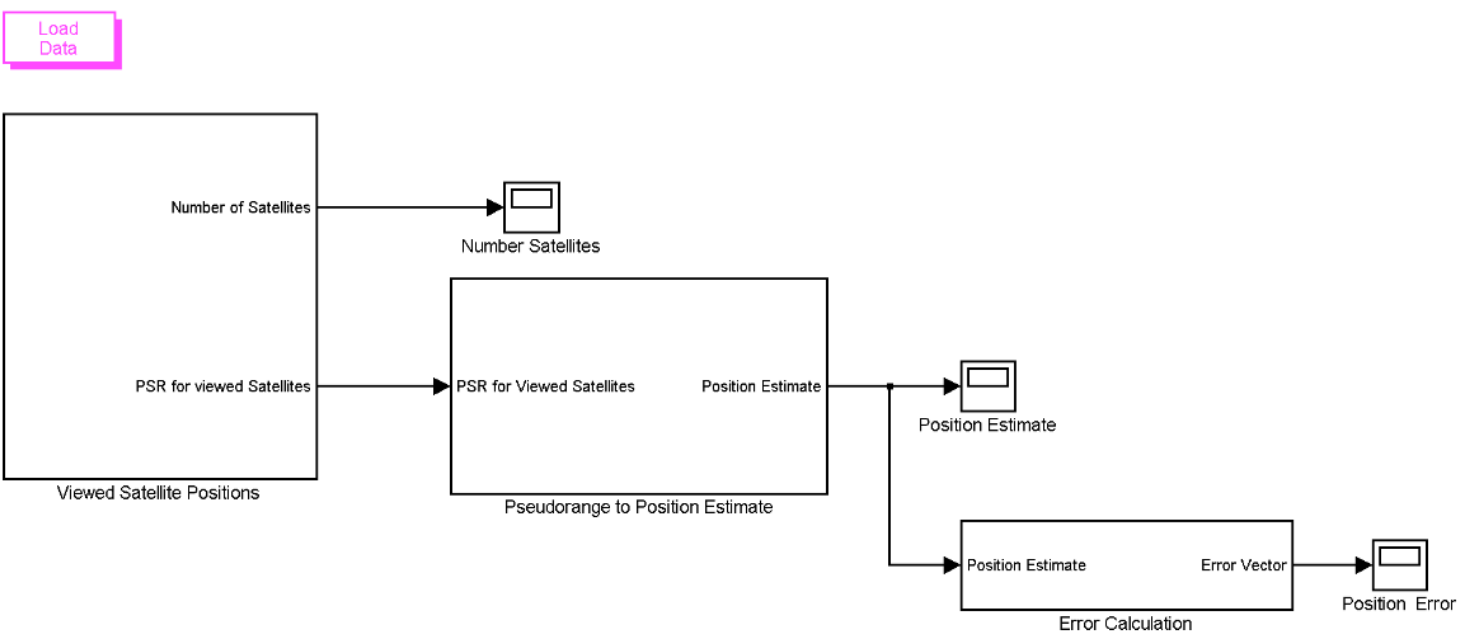

Figure 4.8: GPS data play-back block diagram

The play-back of a GPS receiver's recorded data is useful when determining differences between the GPS receiver's position and velocity estimation algorithm and an individually formulated algorithm. The viewed number of satellites block imports the recorded data and formats the data into the desired format. The next block labeled pseudorange to position estimation is an EKF that provides a position estimate. The position estimate is then fed into the error calculation block. For this thesis this was the estimated true position, this was developed however with the ability to use the reported GPS receiver's position estimate. This tool was used throughout the evaluation process of this thesis project. 


\subsection{Initial Orbit Determination}

For future projects, the use of IGU product data will most likely need the ability to estimate a user's velocity. With this being said the velocity of a satellite will need to be known. However, most IGU product datasets do not include a velocity of the satellites. An initial estimate of the velocity of the satellites will need to be determined before further orbital estimations are performed.

There are multiple methods for calculating a velocity of an unknown orbit. Each of the following methods were used and evaluated. The methods include: Gibbs, Herrick-Gibbs, and Lambert with universal variables. Each of these methods has their respective strengths and weaknesses in the estimation of a satellite's velocity. A brief description about each method is provided, however for more information about these methods see [85].

The Gibbs method is basically vector analysis. This method is formed by knowing three nonzero, coplanar position vectors, which represent three time-sequential vectors of a satellite in orbit. The nonzero position vector assumption is to prevent divisions by zero. The coplanar assumption will be relaxed due to possible observation imperfections. The sequential requirement is important due to in the formation of this method cross products are taken based on the given order. A downside to this method is that it does not perform well with very closely spaced position vectors $[\mathbf{8 5}]$.

The Herrick-Gibbs method is a variation of the Gibbs method. This method requires three sequential position vectors and their observation times. This method uses a Taylor-series expansion to determine the velocity vector at the middle position vector. By using a Taylorseries expansion, this method is not as robust as the Gibbs method. The Herrick-Gibbs method however preforms more accurately with closely spaced position vectors.

Vallado identified that the Herrick-Gibbs is superior of these two methods when the separation angle is less than 1 degree, and the Gibbs method is superior with an angle greater than 5 degrees [85]. The Gibbs method, however, performs more accurately with larger separation angles. Figure 4.9 shows the separation angle between the first and second position vectors throughout a dataset of satellite PRN-1. The angle never goes below 5 degrees or above 6.2 degrees due to the fixed 900 second interval of the dataset. The amount of separation raises the suspicion in which method should be used. 


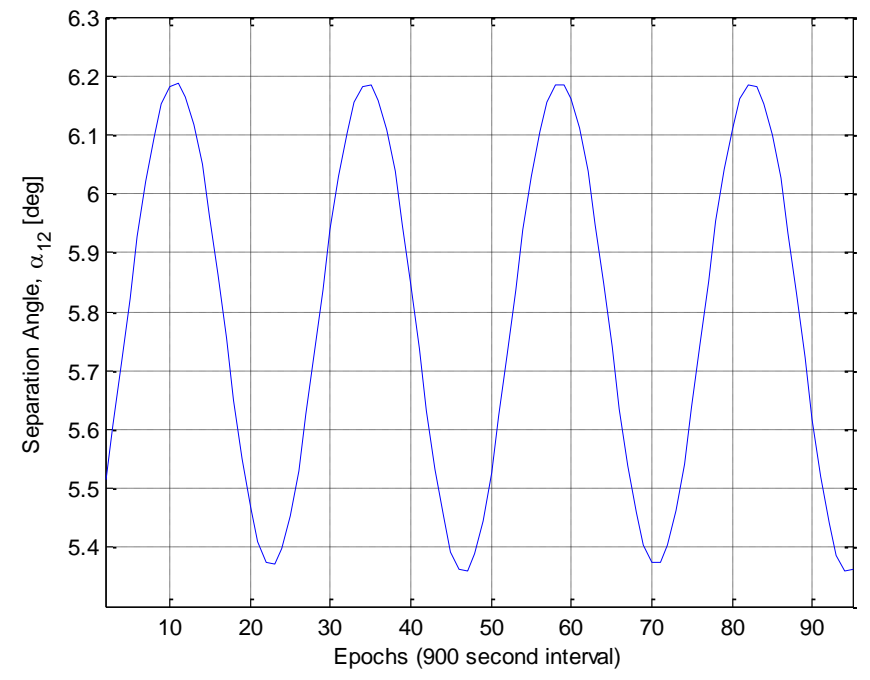

Figure 4.9: PRN-1 satellite's separation angle throughout a dataset

The Lambert with universal variables method is an alteration of Gauss's method. Gauss's method is based on obtaining two independent equations that relate the change in eccentric anomaly, $E$, and the ratio between the swept area of the satellite and the area bounded by position vectors [85]. The only inputs for this method are the position vectors, and the outputs are the velocity components at each position. Therefore, each time epoch has two velocity estimates. Both of these velocity estimates are shown in the figure below to reveal the variation within this method. The following figure is a sample of velocity estimates in the $x$-direction. The $y$ - and $z$-directions have similar trends.

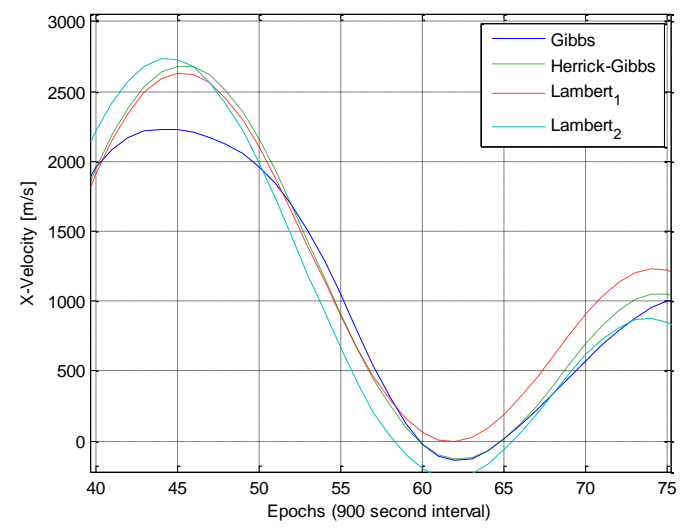

Figure 4.10: Method comparison of $\mathrm{X}$-axis velocity estimates 
Clearly from Figure 4.10, without the use of a truth data, the ability to determine the optimal velocity estimate is difficult. Upon further investigation, another method was also revealed to be applicable. This method extends the broadcasted orbital parameters to calculate the velocity and acceleration of a GPS satellite. This method will be referred to within this thesis as the extended broadcast ephemeris (EBE) method. Derivations of this method include the conventional rotation matrix method and a first-order central difference Taylor series approximation, [52,86]. The EBE method implemented within the MATLAB tools was the rotation matrix method. Although Gibbs, Herrick-Gibbs, and Lambert with universal variables are practical and easily implemented the rotation matrix method was selected because it is the conventional approach. The rotation matrix method assumes Kepler's second law: a radius vector sweeps out equal areas in equal intervals of time. The Taylor series method used by Zhang et al. was reported to be identical to the rotation matrix method and have an accuracy of $\pm 1 \mathrm{~mm} / \mathrm{sec}$ in all axes for a sample dataset [52]. By implementing the rotation matrix method rather than the previously described methods, a precise velocity and acceleration estimate can be obtained.

\subsection{GPS Orbit Interpolation}

The use of precise satellite positions is required at an increased frequency compared to the reported 900 second interval. Therefore, an interpolation strategy that does not corrupt the precision of the data must be employed. A Lagrange interpolator has been used in the past by Defense Mapping Agency (DMA) [87], National Geodetic Survey (NGS) [88], Jet Propulsion Laboratory (JPL) and other organizations [14]. Schenewerk compared polynomial and trigonometric interpolators, and revealed that a 7-term trigonometric interpolator outperformed all other cases in his study [13]. Feng et al. evaluated Lagrange, Chebyshev and trigonometric interpolators, and suggested that trigonometric representation of $24 \mathrm{hr}$. GPS orbits could be developed as an alternative to the Standard Product Version 3 (SP3).

After reading the work of Schenewerk [13] and Feng et al. [14], it was concluded that the best interpolator to use was a trigonometric function. The process that was taken for this study was a combination of the approaches of Ruibo et al. [89], Schenewerk [13], and Feng et al. [14]. When there is an availability of IGR product data for a particular day of interest, the preceding 
and subsequent days are used. This is due to the possibility of errors increasing near the beginning and end of a dataset.

Flight times for the FCSL are usually within the range of 17:00 to 20:00 GPST. For off-line analysis, the following interpolation strategy was developed. Figure 4.11 shows a plot of the position of satellite PRN-9 in ECEF coordinates. The starting time of the interpolation is at 00:00 GPST 10/9/2011 and ends at 23:45 GPST 10/11/2011.

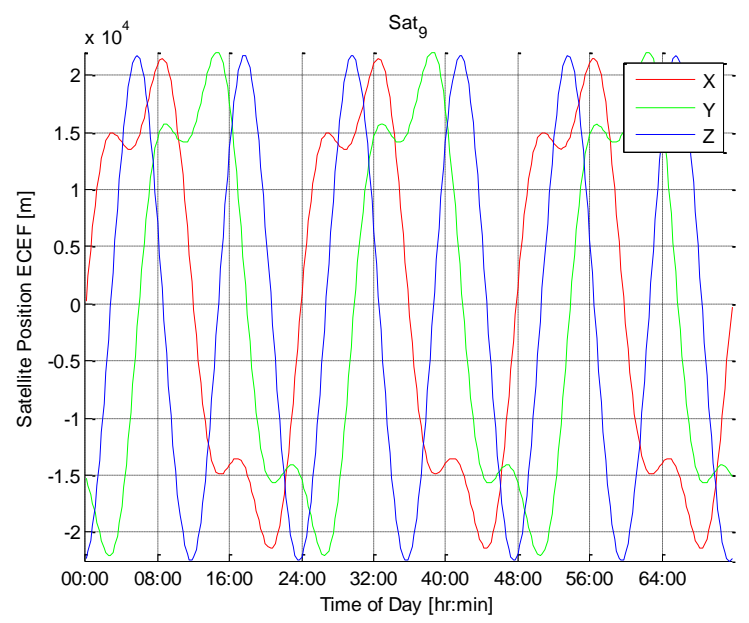

\section{Figure 4.11: ECEF position coordinates from precise ephemeral data}

As used in Ruibo and Xiaoqiang's paper [89], a Fourier series is used to fit the GPS product data. Equation 4.2 is the Fourier series where $C$ is the dependent variable, $t$ is the independent variable, $a_{k}$ and $b_{k}$ are the coefficients, $N$ is the order, and $w$ is the frequency. In this context, $t$ is the time in seconds, $C$ is the $x, y$, and $z$ coordinates in the ECEF frame of the satellite.

$$
C=\sum_{k=0}^{N}\left[a_{k} \cos (k w t)+b_{k} \sin (k w t)\right], k=0,1,2, \ldots, N
$$

Due to this form, $w$ will be a conversion of the ratio of solar to sidereal day, $d \theta / d t$, for the span of days and time scale. This calculation is as follows, the value of $d \theta / d t$ was obtained from the IERS Conventions (2010) [90], which can also be viewed in Appendix A. 


$$
w=2 \pi(d \theta / d t)(1 / 3)=2 \pi(1.00273781191135448 \text { 1/day })(1 / 86400 \mathrm{day} / \mathrm{sec})(1 / 3)
$$

The independent variable was not rescaled such that it would remain in terms of seconds. The number of terms is related to the number of data points. Feng et al. suggested two 20-term trigonometric functions for $12 \mathrm{hrs}$ of GPS ephemeral data [14]. However, this study is using only one function for three days (72 hrs), requiring a factor of 6 to number of terms to properly capture all frequencies of the behavior, therefore 120 terms were used in the Fourier series. The approximation with these specifications resulted in similar plots to Schenewerk [13], and Feng et al. [14], seen in the figure below. This figure is of the $\left|x_{f i t}-x\right|+\left|y_{f i t}-y\right|+\left|z_{f i t}-z\right|$ or the sum of the absolute differences in ECEF for satellite PRN-9.
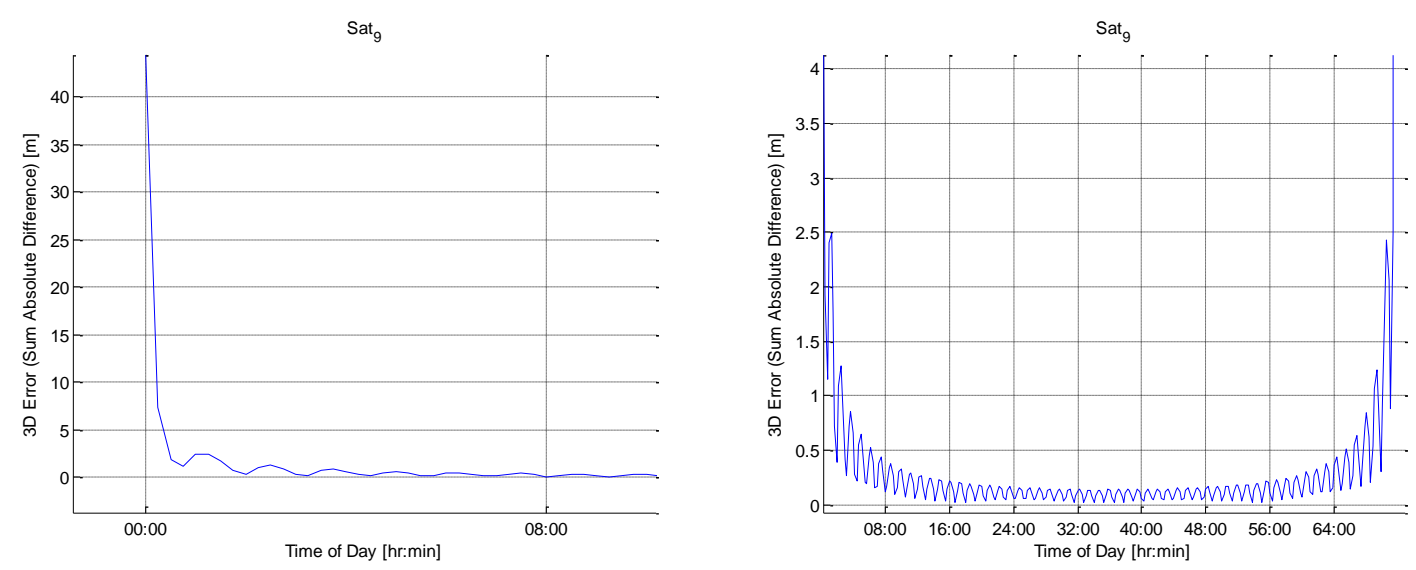

Figure 4.12: Sample of initial interpolation results

The plot on the left of Figure 4.12 is a zoomed in version of the first 8 hours of this interpolation. This reveals divergence of the approximation at the beginning and end of the dataset. This is a similar characteristic of Schenewerk and Feng's results [14,13]. During the interested day (24:00-48:00) the mean error is approximately 0.1 meters. Since the errors are undesirably large during the interested time span, an alteration to the Fourier series was necessary. The frequency was then divided by 2 to limit the available frequencies modeled by half. This is due to the effects of both the orbital motion and the rotation of the coordinate frame being displayed in the ECEF position coordinates. This change causes the frequency, $w$, to be the following: 


$$
w=2 \pi(d \theta / d t)(1 / 3)(1 / 2)=1.215352524451163 \mathrm{e}-5
$$

The results obtained with this adjustment reaffirm the statement of Feng et al. "trigonometric representations of GPS orbits could be developed as an alternative to the SP3 ephemeris format used for IGS services" [14]. Figure 4.13 is the sum of the absolute differences of PRN-9 throughout the three day interpolation, the $y$-axis is in terms of centimeters, and $x$-axis is as it was previously.

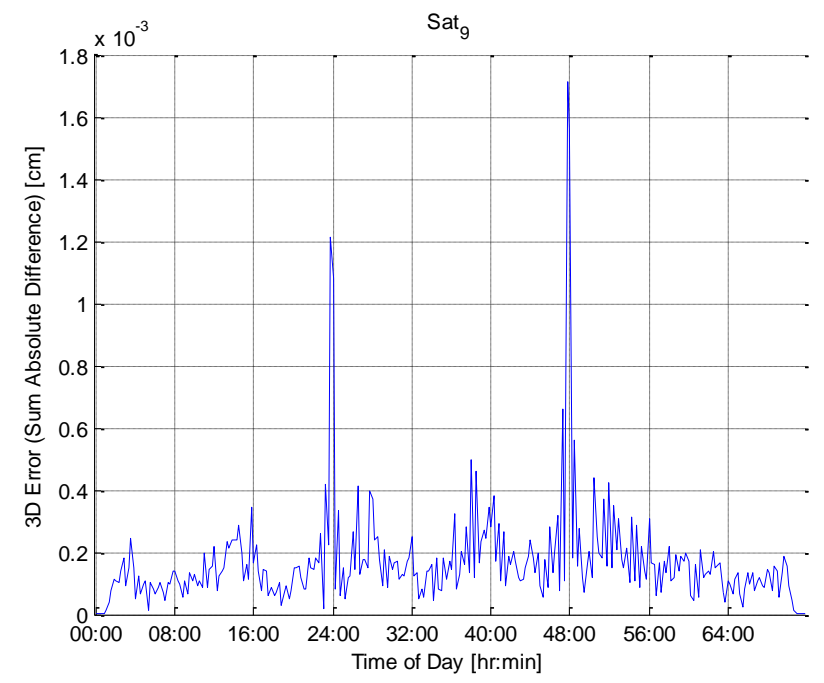

Figure 4.13: Results of the adjusted Fourier series interpolation

The interpolation errors are on a sub-centimeter level throughout the approximation. An interesting increase in error can be viewed at exactly the $24^{\text {th }}$ and $48^{\text {th }}$ hour of the interpolation. This was visible in this dataset on other satellites and also other smaller peaks near the $12^{\text {th }}$ and $36^{\text {th }}$ hours as well. Due to the magnitude of the largest error spike the required accuracy is well within the available accuracy of the product data. Understanding the error spikes is outside the scope of this thesis; however it is concluded this is due to how the IGS products are aligned daily to GPS time (For more information see Kouba's report Section 7.3 [1]). 
For the application of a real-time problem, adjustments need to be made to the interpolation strategy. Due to the nature of nearly circular satellite orbits, a conversion between ECEF coordinates to the ECI frame would result in a smooth sinusoidal curve for each position component. A three dimensional illustration of all GPS satellites within the constellation in the ECI frame throughout the first 12 hours of Oct. 12, 2011 (GPST) is given in Figure 4.14.

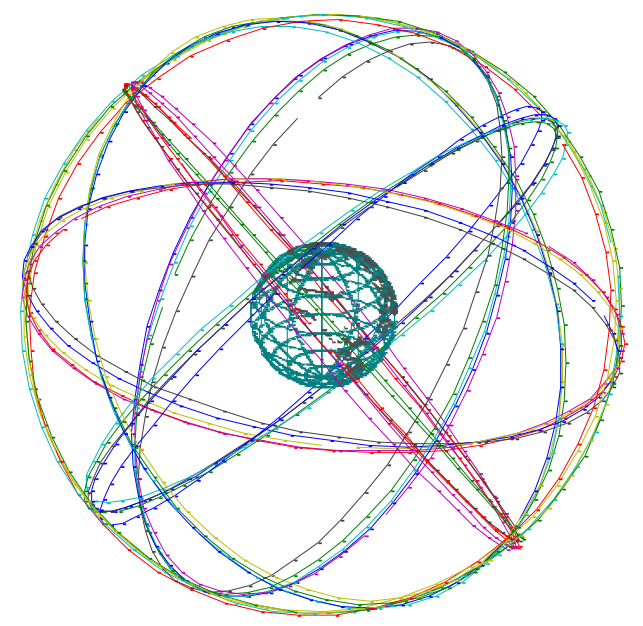

Figure 4.14: GPS contsellation within the ECI frame

Figure 4.14 was provided to visualize the motion of a satellite orbit. The conversion from ECEF to ECI is approximated by removing the Earth's rotation from satellite's position. This is completed by calculating the Julian date, from this the Greenwich Apparent Sidereal Time (GAST) is then determined. The Earth's orientation can be determined from using the GAST. The Earth orientation can then be removed throughout the dataset to convert the ECEF coordinate to ECI. The following text and reference was used for the conversion from ECEF to ECI [91], [92]. Figure 4.15 is an example of a satellite's position throughout a dataset in the ECI coordinate frame. The dataset used is the first available Ultra-rapid product on Oct. 12, 2011 (GPST). 


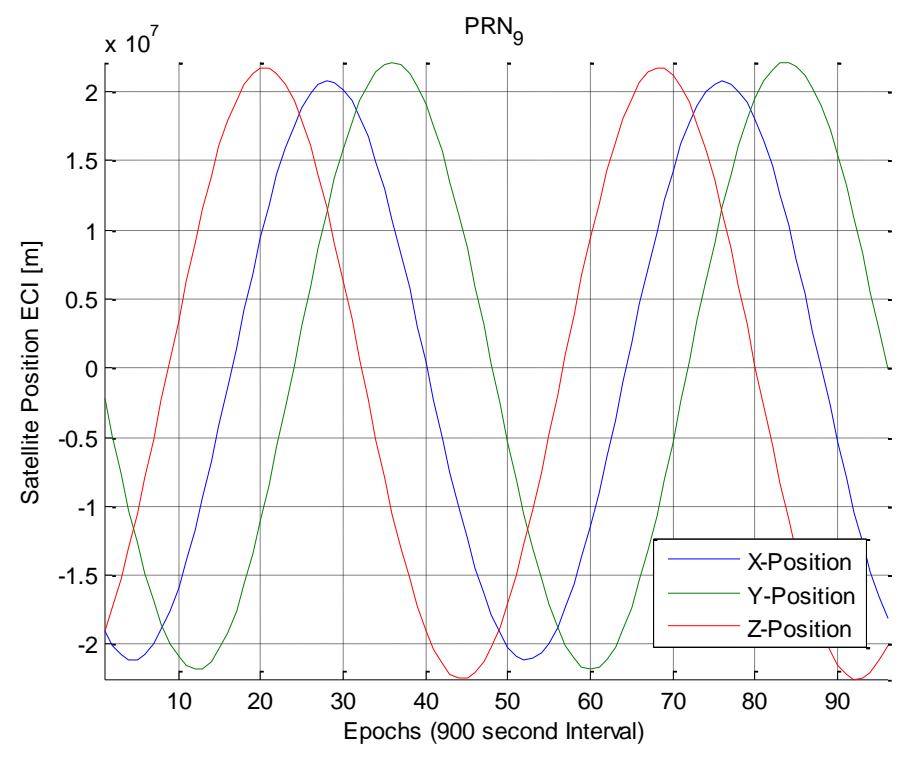

\section{Figure 4.15: Satellite PRN-9 position throughout entire IGU dataset}

Alterations were made to the interpolation strategy such that it would properly be used for the ECI positions. The first of which was changing the frequency, $w$, back to the original solar to sidereal day ratio with the conversion to the appropriate time scale. Also the number of coefficients of the interpolation strategy was decreased. The following figure shows the ECI interpolation strategy's sum of absolute differences for satellite PRN-30 over the entire IGU dataset. This figure is the worst performing satellite interpolation of this dataset; however this still is a representation of the interpolation strategy. The 'worst' interpolation was determined by finding the satellite with the largest cost function value. The cost function evaluated is: $J=\int\left|x_{f i t}-x\right|+\left|y_{f i t}-y\right|+\left|z_{f i t}-z\right| d t$. 


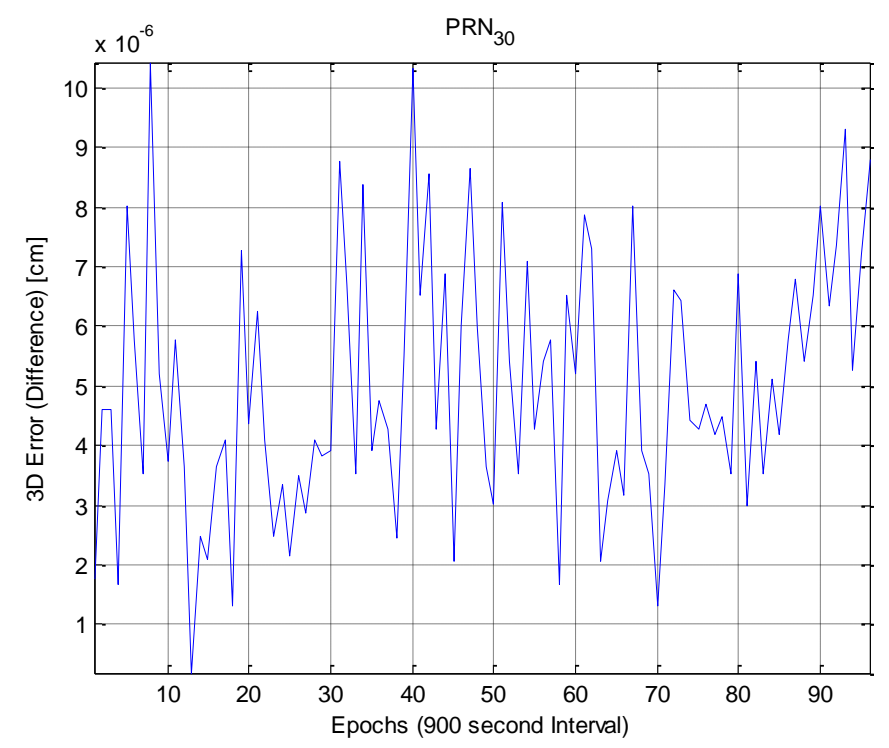

Figure 4.16: ECI interpolation of Satellite PRN-30 performance

Due to the level of precision of the product data and of these interpolation strategies, the precision of the interpolated information is assumed to have the same accuracy. To further evaluate the usefulness of this information, a comparison between a position estimation algorithm using this interpolated information and the same position estimation algorithm using recorded data is evaluated in the next chapter. 


\section{Chapter 5 : Estimation Comparison}

The created MATLAB tools information is evaluated based on a real-time application. A comparison is presented between an EKF with using recorded GPS data and an EKF that uses the MATLAB tools information. The comparison will be conducted by using two different sets of data of a static GPS receiver for approximately 15 minutes of time. As stated previously, the collection of this data was completed with the use of the Green PAC aircraft.

First, the use of the BESTXYZ packet information is discussed. Then the evaluation of the true position of the static GPS receiver is then described. Next, the satellite position estimation from the use of the SATXYZ packet is discussed. The TIME packet information is also briefly described. Next, the formulated EKF is described and thereafter an evaluation of the created MATLAB tools information is presented. This evaluation compares the results of an EKF that uses recorded GPS data and the same EKF using a portion of the created MATLAB tools information.

\subsection{True Position Estimation}

As mentioned before, the Green PAC aircraft records the BESTXYZ, SATXYZ and TIME NovAtel GPS data logs. The BESTXYZ packet includes the position, velocity, and the standard deviations of each of these measurements. The position estimation from this packet is used to evaluate the approximate true position of the GPS receiver. By assuming the GPS position has a normal distribution for each component of measurement, the mean of each component is used to approximate the true location of the GPS receiver. A refinement of data is conducted to more accurately approximate the true position. The use of the standard deviations of the position estimates is used to determine a threshold or percentage in which high noise measurements will be removed from the dataset.

The first step of this process was to calculate an approximation of the circular error probable (CEP). The CEP is the radius of a circle in which $50 \%$ of the values occur. A commonly used approximation was taken from Maybeck's text in Chapter 6.11 of the follow reference [93]. Using the reported standard deviations within the BESTXYZ the following equation was used to determine the CEP throughout the set of data $C E P=0.563 \sigma_{x}+0.614 \sigma_{y}$. A threshold was determined for each dataset to remove any outliers. The maximum percentage of the outliers 
removed was less than $2 \%$. The following figure is of the calculated CEP of the raw datasets and their respective CEP threshold.
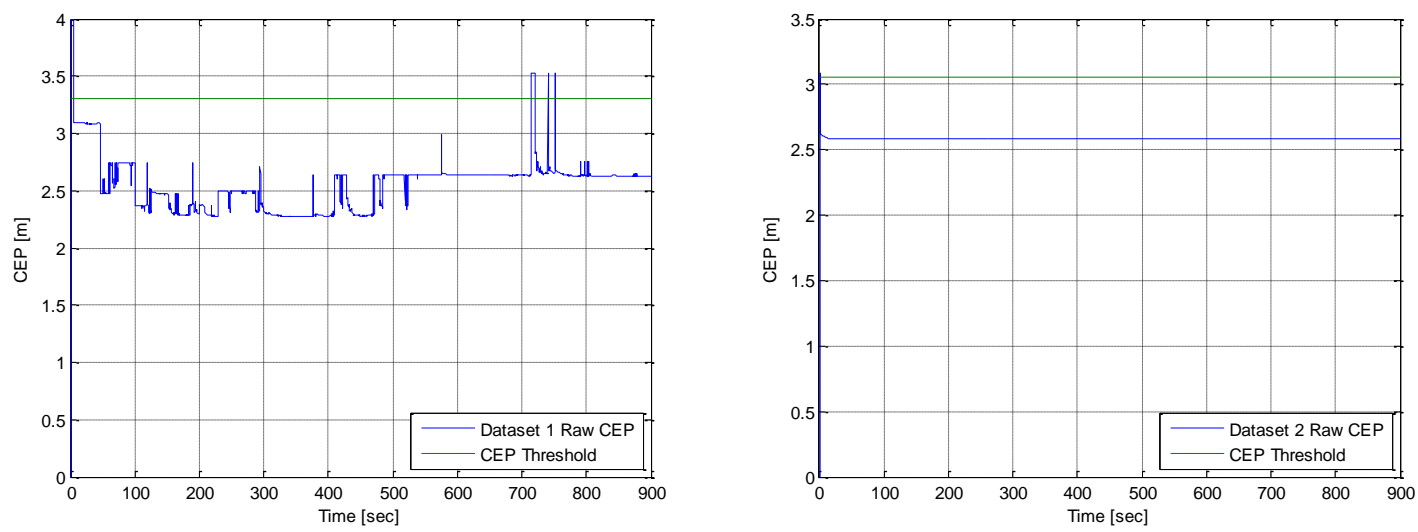

Figure 5.1: CEP thresholds for both Datasets

After the refinement of the BESTXYZ data, the mean was determined. The following scatter plots show the raw data in blue-dots, refined data in black-crosses, and the estimated true position is a red asterisk. In the figure below, the $x$ - and $y$-axes are the local coordinate frame $x$ and $y$-components. The radius of a circle at the true position, containing the position estimate with $95 \%$ probability was also placed on this plot as well. These circles are referred to as the R95 the raw data R95 is a dashed red line, and the R95 for the refined data is a solid red line. Even if the precision of the assumed true estimated location is off the results will only be biased.
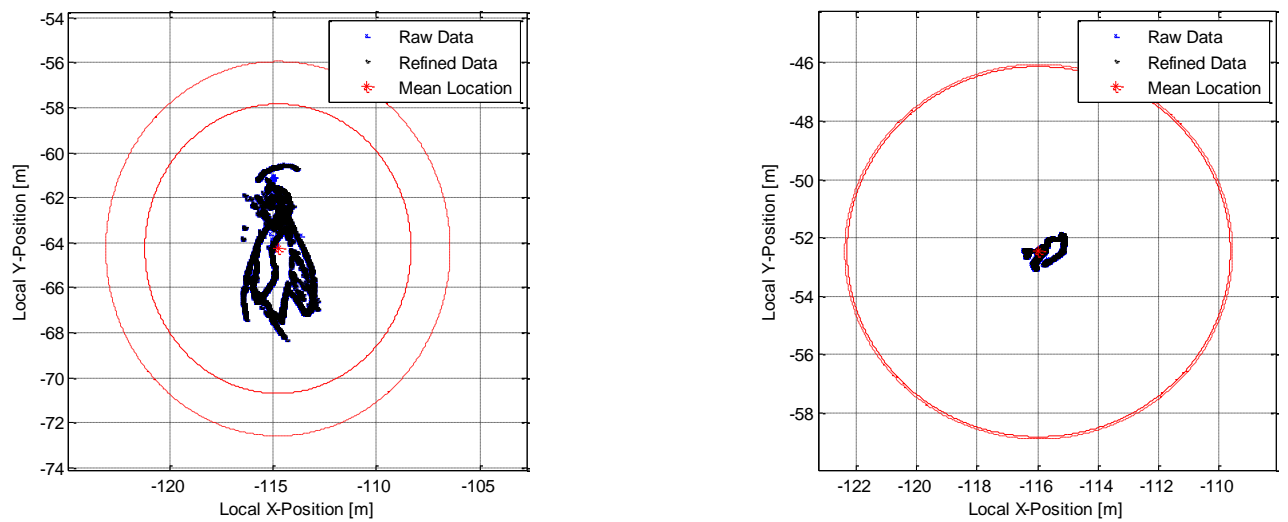

Figure 5.2: True Position Estimation

The reason for selecting these two datasets was due to the number of satellites used in obtaining the solution of the BESTXYZ position estimate. Figure 5.3 is of the viewed number of 
satellites and the number of satellites used for the solution of the position estimate. For both plots the solution number of satellites and viewed number of satellites match exactly.
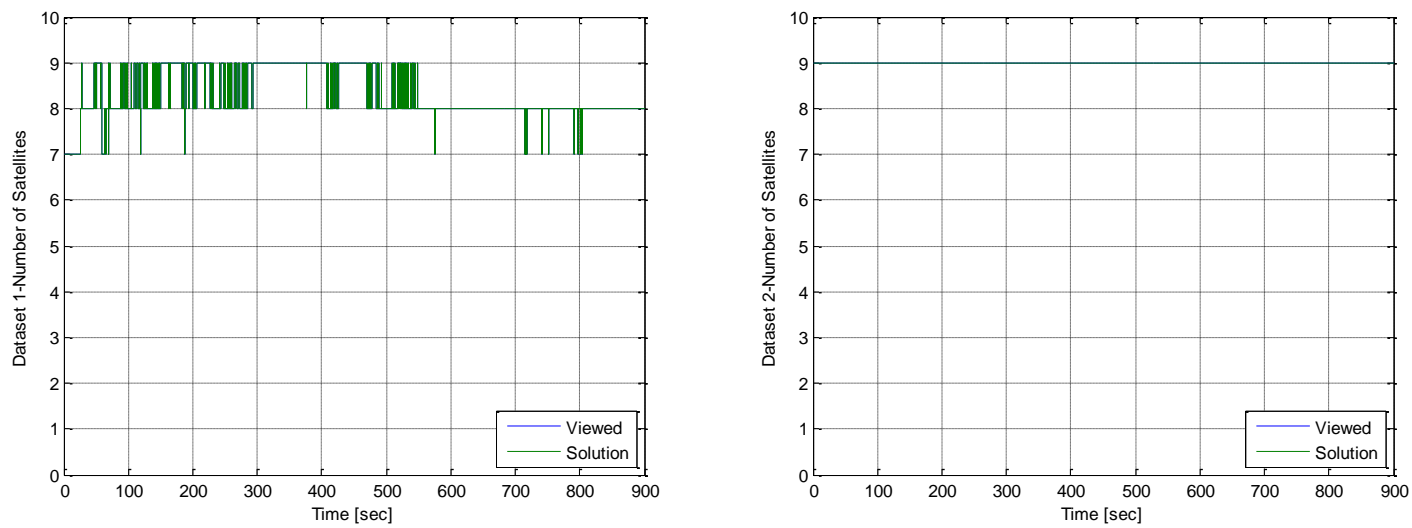

Figure 5.3: Number of satellites throughout datasets

These specific datasets were selected because of this exact match of viewed and solution number of satellites. This is because it would be difficult to determine which satellites were used for the position estimation if these did not match exactly. Although Dataset-2 the BESTXYZ packet revealed nine satellites in view a tenth satellite was observed within the SATXYZ packet. The cause for this is unknown, however conclusions that the satellite was below a viewing threshold could be considered. Other reasons will be discussed in Section 5.4.

\subsection{Satellite Position from Recorded NovAtel Data}

The EKF that will use recorded data will primarily use the SATXYZ packet. NovAtel's SATXYZ packet has a dynamic packet length, which changes with respect to the number of observed GPS satellites. This caused some initial data recording issues due to the size and rate at which the data was sent. This was mitigated by changing the rate at which the GPS receiver would send the packet information. Even after this initial mitigation, further steps needed to be conducted to allow the use of this information. The following table from NovAtel's manual describes in detail the SATXYZ's protocol. 
Table 5.1: NovAtel's SATXYZ Packet [69]

\begin{tabular}{|c|c|c|c|c|c|}
\hline Field \# & Field type & Data Description & Format & $\begin{array}{l}\text { Binary } \\
\text { Bytes }\end{array}$ & $\begin{array}{l}\text { Binary } \\
\text { Offset }\end{array}$ \\
\hline 1 & SATXYZ header & Log header & & H & 0 \\
\hline 2 & Reserved & & Double & 8 & $\mathrm{H}$ \\
\hline 3 & \#sat & $\begin{array}{l}\text { Number of satellites with Cartesian } \\
\text { information to follow }\end{array}$ & Ulong & 4 & $\mathrm{H}+8$ \\
\hline 4 & PRN/slot & $\begin{array}{l}\text { Satellite PRN number of range } \\
\text { measurement (GPS: } 1-32 \text { and } \\
\text { SBAS: } 120 \text { to } 138 \text {. For GLONASS, } \\
\text { see Section } 1.3 \text { on page } 29 \text {.) }\end{array}$ & Ulong & 4 & $\mathrm{H}+12$ \\
\hline 5 & $x$ & Satellite $X$ coordinates (ECEF, m) & Double & 8 & $\mathrm{H}+16$ \\
\hline 6 & $\mathrm{y}$ & Satellite $Y$ coordinates (ECEF, $m$ ) & Double & 8 & $\mathrm{H}+24$ \\
\hline 7 & $z$ & Satellite $Z$ coordinates $(E C E F, m)$ & Double & 8 & $\mathrm{H}+32$ \\
\hline 8 & clk corr & Satellite clock correction (m) & Double & 8 & $\mathrm{H}+40$ \\
\hline 9 & ion corr & Ionospheric correction (m) & Double & 8 & $\mathrm{H}+48$ \\
\hline 10 & trop corr & Tropospheric correction (m) & Double & 8 & $\mathrm{H}+56$ \\
\hline 11 & \multirow{2}{*}{\multicolumn{2}{|c|}{ Reserved }} & Double & 8 & $\mathrm{H}+64$ \\
\hline 12 & & & Double & 8 & $\mathrm{H}+72$ \\
\hline 13 & \multicolumn{5}{|c|}{ Next satellite offset $=\mathrm{H}+12+(\#$ sat $\times 68)$} \\
\hline variable & $x x x x$ & 32-bit CRC (ASCII and Binary only) & Hex & 4 & $\begin{array}{l}\mathrm{H}+12+ \\
\text { (\#sat } \mathrm{x} \\
68)\end{array}$ \\
\hline variable & [CR][LF] & Sentence terminator (ASCII only) & - & - & - \\
\hline
\end{tabular}

The SATXYZ packet will be used to evaluate a baseline performance of the EKF formulation. The use of a satellite's position and tropospheric and ionospheric correction terms will be used with in the EKF. It was observed that an update rate of $10 \mathrm{~Hz}$ of this information yielded the highest update rate with a small number of improperly recorded or missing updates. Usual values of a missing packet would be approximately zero, and for an improperly recorded packet random values were viewed. Since these corrupted measurements the recorded information will need to be refined. Due to the nature of why these measurements are corrupted only short intermittent sections of the datasets were needed to be manipulated. Approximately one to three sequential measurement samples were seen to be corrupted every twenty seconds.

However the frequency and duration of sequential corrupted measurements was not predictable therefore a more intuitive strategy was determined for their identification and removal. The determination of corrupted measurements was completed by taking a numerical derivative of a satellites position measurement. This would yield an approximation of the satellite's velocity. Since GPS satellites have nearly circular orbits they have approximately a constant velocity throughout their orbit.

Corrupted measurements were determined by large changes in a satellite's velocity. The determination of a "large" change was multiple orders of magnitude larger than the nominal case. After these measurements were identified a simple interpolation strategy was deemed appropriate due to the update frequency and the change in a satellites position for short intervals. 
This same interpolation was applied to the tropospheric and ionospheric correction terms as well. This is also applicable in this case since the GPS receiver is static, the tropospheric and ionospheric correction terms will be nearly constant. A sample of a dataset is included below; this is of satellite PRN-12 ECEF y-position for approximately 600 samples or an equivalent 60 seconds.
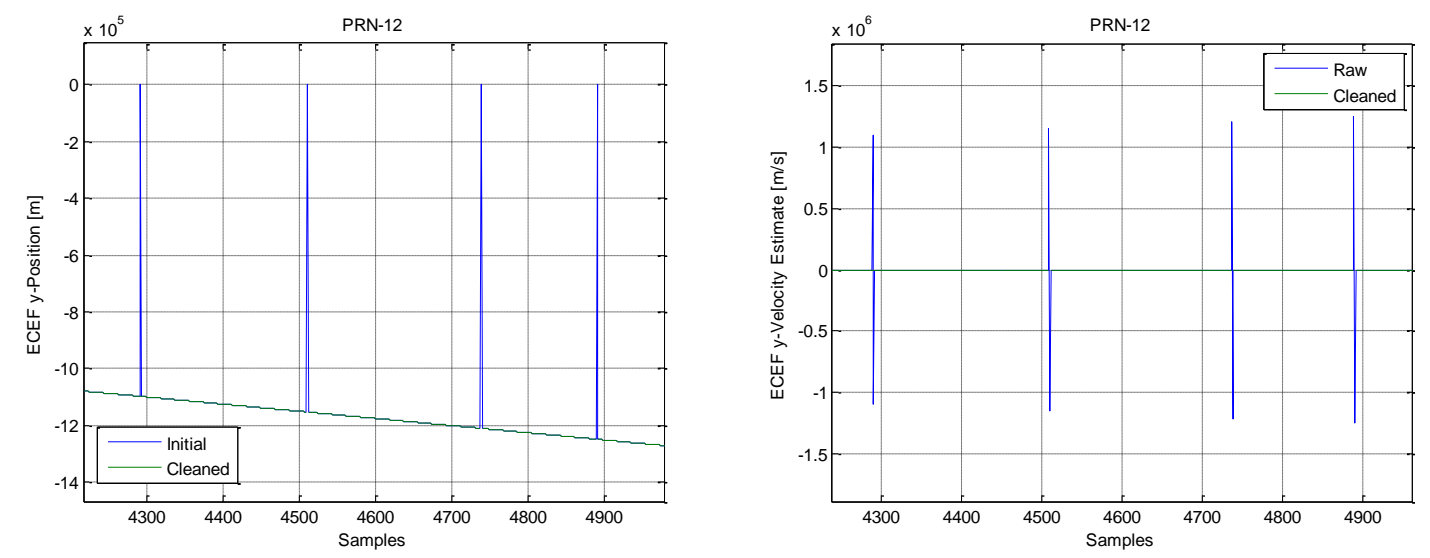

Figure 5.4: Sample of a refinement of SATXYZ dataset

An atmospheric correction term, $\varepsilon$, was calculated by the summation of the ionospheric correction term and tropospheric correction terms. Both measurement sources that will be evaluated will use this summed atmospheric correction term when calculating the pseudoranges for each satellite. Including this term is an approximation for the observed atmospheric errors. 
The inclusion of the TIME packet was used to accurately correlate information to the UTC time frame. The TIME packet also includes the clock offset for the GPS receiver from the GPST. The TIME packet has the following architecture:

Table 5.2: NovAtel's TIME Packet [69]

\begin{tabular}{|c|c|c|c|c|c|}
\hline Field \# & Field type & Data Description & Format & $\begin{array}{l}\text { Binary } \\
\text { Bytes }\end{array}$ & $\begin{array}{l}\text { Binary } \\
\text { Offset }\end{array}$ \\
\hline 1 & $\begin{array}{l}\text { TIME } \\
\text { header }\end{array}$ & Log header & & $\mathrm{H}$ & 0 \\
\hline 2 & $\begin{array}{l}\text { clock } \\
\text { status }\end{array}$ & $\begin{array}{l}\text { Clock model status (not including current } \\
\text { measurement data), see Table } 54 \text { on page } 269\end{array}$ & Enum & 4 & $\mathrm{H}$ \\
\hline 3 & offset & $\begin{array}{l}\text { Receiver clock offset, in seconds from GPS time. A } \\
\text { positive offset implies that the receiver clock is } \\
\text { ahead of GPS time. To derive GPS time, use the } \\
\text { following formula: GPS time = receiver time - offset }\end{array}$ & Double & 8 & $\mathrm{H}+4$ \\
\hline 4 & offset std & Receiver clock offset standard deviation. & Double & 8 & $\mathrm{H}+12$ \\
\hline 5 & utc offset & $\begin{array}{l}\text { The offset of GPS time from UTC time, computed } \\
\text { using almanac parametres. UTC time is GPS time } \\
\text { plus the current UTC offset plus the receiver clock } \\
\text { offset: UTC time = GPS time + offset + UTC offset }\end{array}$ & Double & 8 & $\mathrm{H}+20$ \\
\hline 6 & utc year & UTC year & Ulong & 4 & $\mathrm{H}+28$ \\
\hline 7 & utc month & UTC month (0-12) a & Uchar & 1 & $\mathrm{H}+32$ \\
\hline 8 & utc day & UTC day $(0-31)^{a}$ & Uchar & 1 & $\mathrm{H}+33$ \\
\hline 9 & utc hour & UTC hour $(0-23)$ & Uchar & 1 & $\mathrm{H}+34$ \\
\hline 10 & utc min & UTC minute (0-59) & Uchar & 1 & $\mathrm{H}+35$ \\
\hline 11 & utc ms & UTC millisecond $(0-60999)^{b}$ & Ulong & 4 & $\mathrm{H}+36$ \\
\hline 12 & utc status & $\begin{array}{ll}\text { UTC status } & \\
& =\text { Invalid } \\
1 & =\text { VValid }^{\mathrm{c}} \\
2 & =\text { Waming }^{\mathrm{c}}\end{array}$ & Enum & 4 & $\mathrm{H}+40$ \\
\hline 13 & $x \mathrm{xxx}$ & 32-bit CRC (ASCII and Binary only) & Hex & 4 & $\mathrm{H}+44$ \\
\hline 14 & [CR][LF] & Sentence terminator (ASCII only) & - & - & - \\
\hline
\end{tabular}

This information is not used within the EKF however for other purposes with respect to the MATLAB tools the inclusion of this information is useful. Within every NovAtel header section of a message the millisecond of the week is provided, however this is not in terms of the accuracy in which some problems require. For a more accurate time measurement the TIME packet should be used. 


\subsection{Extended Kalman Filter Formulation}

The EKF that was used to evaluate the effectiveness of using the refined MATLAB tools IGU product data was formulated by following Chapter 9.2 of Kaplan's textbook [43]. The states of this EKF formulation are the position of the GPS receiver and clock bias, therefore the state vector is the following: $\mathbf{x}=\left[\begin{array}{llll}x_{E C E F} & y_{E C E F} & z_{E C E F} & t_{u}\end{array}\right]^{T}$. The receiver clock bias is required due to the accuracy of the GPS receiver clock, for more information about this review Section 2.1.3. Units for the states are meters for position and seconds for the clock bias. Since this EKF does not have velocity as a state, the transition matrix, $A$, is just an identity matrix.

The measurement matrix relates the observation to the state vector. For this formulation the measurement matrix is the range between the satellite and GPS receiver. An estimated range, $r_{j}$, from the GPS receiver and a satellite position will need to be determined through the use of the follow equations below. Within these equations $j$ denotes the $j$ th satellite, $\left(x_{u}, y_{u}, z_{u}\right)$ are the GPS receiver's position, $\left(x_{j}, y_{j}, z_{j}\right)$ are the satellite position components in the ECEF frame.

$$
\begin{array}{cc}
r_{x}=\left(x_{j}-x_{u}\right) & \mathbf{5 . 1} \\
r_{y}=\left(y_{j}-y_{u}\right) & 5.2 \\
r_{z}=\left(z_{j}-z_{u}\right) & 5.3 \\
r_{j}=\sqrt{r_{x}^{2}+r_{y}^{2}+r_{z}^{2}} & \mathbf{5 . 4}
\end{array}
$$

The pseudorange will be calculated by Equation 5.5. Within in this equation $\rho$, is the pseudorange, $c$, is the speed of light, $t_{u}$ is the clock bias, and $\varepsilon$ is an atmospheric correction term.

$$
\rho_{j}=\sqrt{\left(x_{j}-x_{u}\right)^{2}+\left(y_{j}-y_{u}\right)^{2}+\left(z_{j}-z_{u}\right)^{2}}+c t_{u}+\varepsilon
$$

The measurement or observation vector has a length of the number of viewed satellites and the elements of this are the pseudoranges for each satellite. The number of rows of $H_{k}$ is equal to the number of viewed satellites. Therefore, for just an example if there was only one satellite in view; the following would be the observation matrix. The elements within this matrix are the range components normalized by the range. 


$$
H_{k}=\left[\begin{array}{llll}
\frac{r_{x}}{r_{j}} & \frac{r_{y}}{r_{j}} & \frac{r_{z}}{r_{j}} & c
\end{array}\right]
$$

The initialized state covariance matrix, $P$, was assumed to be: $P=\operatorname{diag}\left(400,400,400,10^{10}\right)$ which has the same associated values of Kaplan's formulation in Chapter 9.2.4.4 of this textbook [43]. The initialized state matrix, $\hat{\mathrm{x}}_{0}=\left[10^{6}, 10^{6}, 10^{6}, 10^{-3}\right]^{T}$, this was assumed to reveal that the EKF formulation converges. This initialization error is discussed further later in this chapter.

Within the process noise covariance matrix, $Q$, the position elements are computed from unmodeled acceleration variance, and the clock bias is computed from the un-modeled clock variance. These variances will be assumed to be the same as a commercial-grade C/A code receiver, this is the same as Kaplan's formulation [43]. The process noise covariance matrix is given by:

$$
Q=\operatorname{diag}\left[\frac{d T^{3}}{3}\left(\sigma_{n}^{2}\right), \frac{d T^{3}}{3}\left(\sigma_{n}^{2}\right), \frac{d T^{3}}{3}\left(\sigma_{n}^{2}\right), \frac{d T^{3}}{3}\left({\sigma_{t_{u}}}^{2}\right)\right]
$$

where $d T$ is the sample time, and $\sigma_{n}$ the standard deviation of the state noise, and $\sigma_{t u}$ is the standard deviation of the clock bias. The sample time is 0.1 seconds, the assumed $\sigma_{n}$ is 0.01 meters, and $\sigma_{t u}$ is assumed to be $10^{-5}$ meters. For this formulation, the measurement noise matrix, $R$, has the same number of rows and columns as the viewed number of satellites. The assumed $\sigma_{\rho}$, or standard deviation of the pseudorange measurements, is again the same as Kaplan's formulation: $\sigma_{\rho}=1$.

A brief description of the steps taken by this EKF formulation will be described now. The first step is to predict the states:

$$
\hat{x}_{k \mid k-1}=A_{k-1} \hat{x}_{k}
$$

Then the extrapolated error covariance with added process noise is computed: 


$$
P_{k \mid k-1}=A_{k-1} P_{k-1} A_{k-1}^{T}+Q_{k-1}
$$

The pseudorange is determined for each viewed satellite; this formulates the measurement vector, $z$. The appropriate size of the measurement noise matrix, $R$, is determined and calculated. The predicted pseudorange measurements are calculated and the observation matrix, $H$, is determined as well. The Kalman gain matrix, $K$, can then be computed:

$$
K_{k}=P_{k \mid k-1} H_{k}^{T}\left(H_{k} P_{k \mid k-1} H_{k}^{T}+R_{k}\right)^{-1}
$$

The estimated state vector is then updated:

$$
\hat{x}_{k}=\hat{x}_{k \mid k-1}+K_{k}\left[z_{k}-h\left(\hat{x}_{k \mid k-1}, 0\right)\right]
$$

Thereafter the covariance of the new estimate is then updated:

$$
P_{k}=\left(I-K_{k} H_{k}\right) P_{k \mid k-1}
$$




\subsection{Evaluation of the Use of MATLAB Tools Information}

This evaluation is conducted through a comparison between an EKF that uses two different measurement sources. The first measurement source is from the recorded SATXYZ information that has been refined such that erroneous measurements have been eliminated. The second measurement source is the satellite positions created from the developed MATLAB tools. Two different datasets were selected due to their variation in the viewed number of satellites and solution number of satellites. A brief discussion follows about how each of these measurement sources were created.

The first measurement source is a refined set of recorded SATXYZ data. This is referred to as the baseline estimation. Since the formulated EKF does not incorporate any sort of fault tolerance with respect to erroneous measurements, the refinement of this recorded data was required. The erroneous measurements were identified by calculating a numerical derivative of the satellites position; this is an approximation of the satellites velocity. Due to the nearly circular orbit of a GPS satellite, large changes in the velocity identify the erroneous measurements. The replacement of these measurements was completed through a simple interpolation.

The second measurement source was created by multiple tools developed for this thesis. This is referred to as the created MATLAB tools measurement (CMTM). Initially the appropriate date and time was determined and the desired predicted IGU product data was imported into MATLAB. The precise ephemerides for the viewed satellites was then converted to the ECI frame and interpolated with the second strategy described in Section 4.3. This Fourier series interpolation was conducted for same time as the recorded SATXYZ data. The GPS satellite positions are then transformed back to the ECEF frame.

As revealed in Section 5.1, Dataset-1 has a higher standard deviation in the number of viewed satellites. This dataset is utilized to investigate the effects of poorly positioned satellites on the receiver position estimation.

Figure 5.5 illustrates the geometry of the satellite constellation at the midpoint of Dataset-1. The approximated true position of the GPS receiver is the blue asterisk, the satellite positions are the black circles, and the multicolored sphere is a representation of the Earth. 

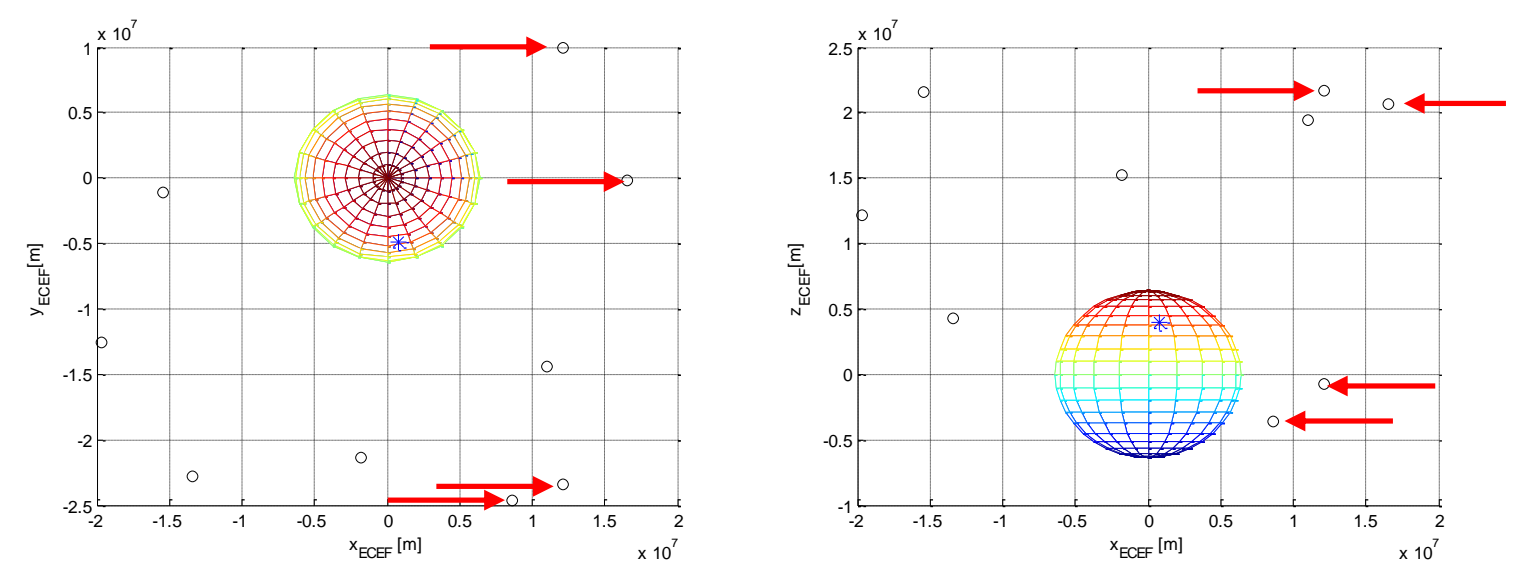

Figure 5.5: Orthogonal views of Dataset-1 constellation geometry

The positions of the identified four satellites in the above figure reveal these are not in an ideal position to increase the precision of the position estimate. The variability of the viewed number of satellites in this dataset is due to most likely these satellites. Two of these are easily identified since they are practically in the earth's shadow and the other two are subjected to larger ionospheric errors.

The quantization of error between the measurement sources will be evaluated based upon the $L^{2}$-Norm of the difference between the estimated position and approximated true position of the GPS receiver. This intuitively is the radius of the estimated position error or $r_{\text {err }}$. This is calculated by the following equation:

$$
r_{\text {err }}=\sqrt{\left(x_{\text {true }}-x_{\text {est }}\right)^{2}+\left(y_{\text {true }}-y_{\text {est }}\right)^{2}+\left(z_{\text {true }}-z_{\text {est }}\right)^{2}}
$$

Qualitatively the trend of radius error ideally would go to zero, however this will not be possible since the EKF is only using pseudorange measurements. As previously shown in Section 2.1.5, the calculation of a satellite's position is determined by the navigation message of a satellite. Within this navigation message, clock information for each satellite is also included. These clock parameters include: clock bias, clock drift, and clock drift rate. These parameters and the associated errors that are accrued without the use of this information are significant. However, the estimation performance of this EKF formulation is not being evaluated. Only the 
difference between the use of the baseline estimation and the CMTM estimation is being evaluated.

The large initialized position error can be viewed within the radius of estimated position error plots. The first time step has a radius error of approximately $10^{6}$ meters within approximately 20 seconds the EKF estimate converges. This demonstrates that this formulation converges in an acceptable amount of time. NovAtel reports for the OEMV-1 a typical time to first fix value of 'cold-start' and 'hot-start' of 60 and 35 seconds respectively [94]. Their definition of a 'coldstart' is when there is no almanac or ephemerides and no approximate position or time, and a 'hot-start' is when there is almanac and recent ephemerides saved and approximate position and time entered.

For Dataset-1 the baseline estimation performs moderately well. An interesting result can be viewed in the figure below. The inclusion of the $9^{\text {th }}$ satellite causes the estimation performance to drift. Once the $9^{\text {th }}$ satellite is no longer in view the estimation performance greatly increases.

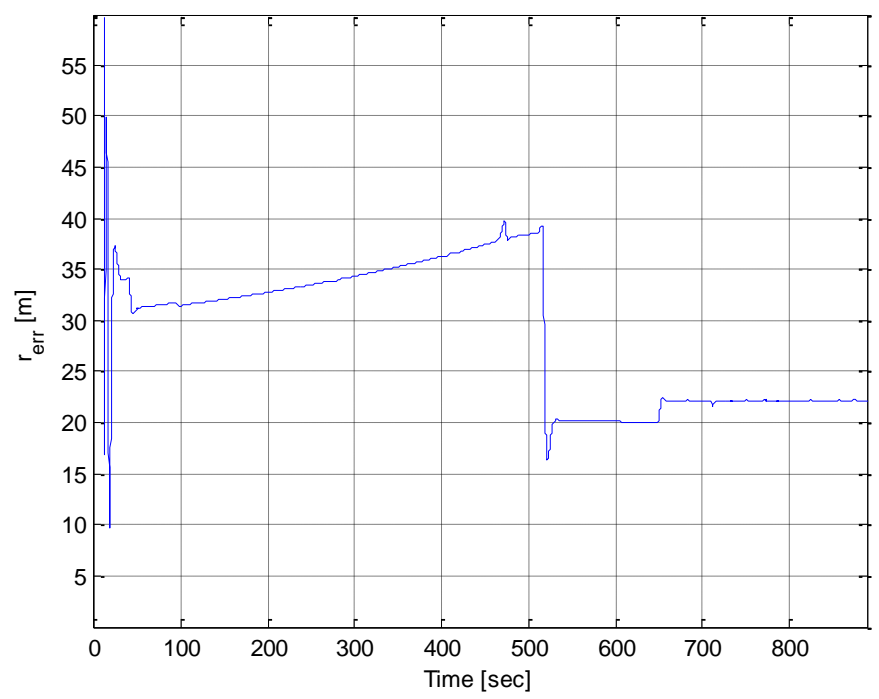

Figure 5.6: Radius of estimated position error for the Baseline estimation of Dataset-1

For this case with a less than desirable satellite geometry, the estimation performance still performs well. By looking at Figure 5.7 a difference between the baseline and CMTM estimation error can be viewed. This difference is an appropriate amount, since this is directly correlated to 
the differences in satellite positions. The difference in the satellite position measurements were assumed to be within a 5-10 meter range. An approximate difference between these two radius errors an average improvement of ten meters can be assumed. This result reveals that the use of the CMTM in a real-time application increases the accuracy of the position estimation.

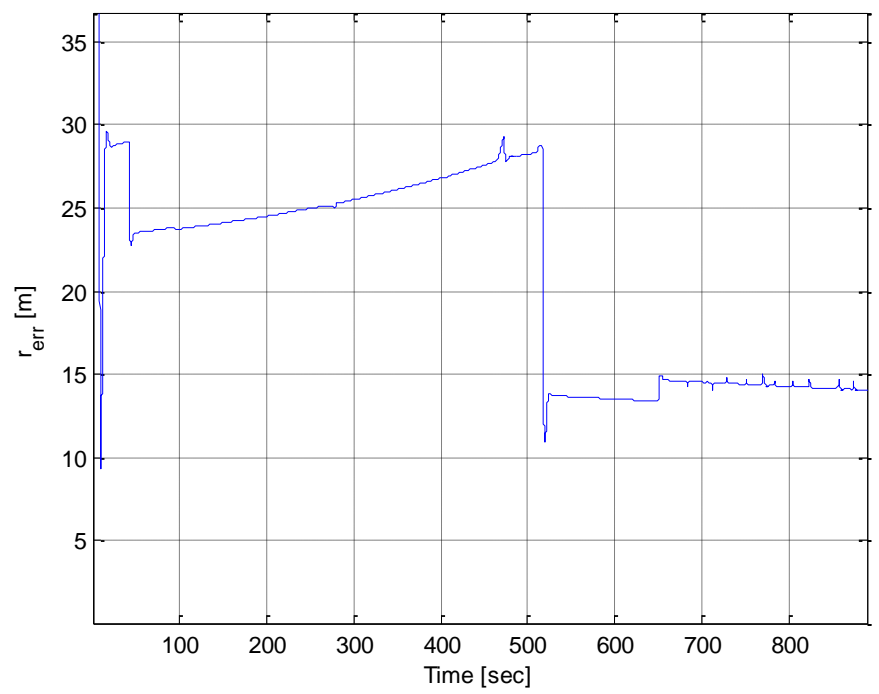

Figure 5.7: Radius of estimated position error for the CMTM estimation of Dataset-1

Once again as for the baseline estimation the CMTM estimation performs similarly. Just as before once the $9^{\text {th }}$ satellite is no longer in view of the GPS receiver the position estimation greatly improves. The improvement of the estimation is only based upon the addition of precise satellite positions created by the MATLAB tools. This reveals that the real-time application of this information is valid and useful.

As stated previously, there were four satellites that were suspected of not being positioned ideally. By the removal of these satellites, the estimation performance increases for both measurements. Figure 5.8 and Figure 5.9 reveal the estimation error, and since the viewed number of satellites decreased by four this inadvertently increases the convergence time of the algorithm. Even though the convergence time increases, once four satellites are in view the position estimation is approximately 10 meters better during 100-550 seconds of time. However, during the end of the dataset the estimation has a slightly higher error than when all satellites 
were used. The difference between the measurement sources error is approximately five meters which is an error reduction of $\sim 25 \%$ by using the CMTM.

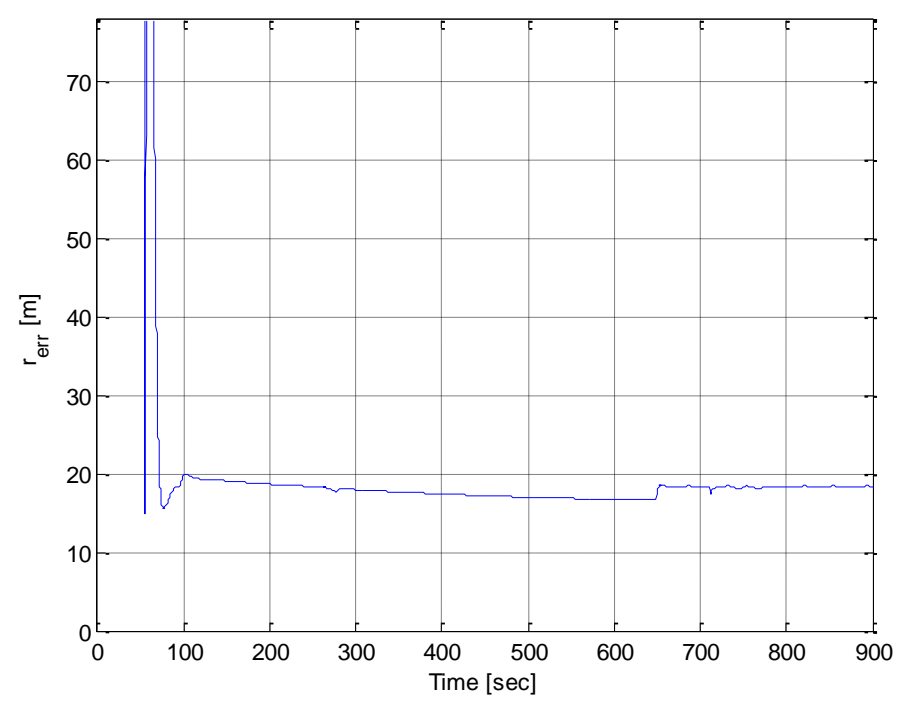

Figure 5.8: Radius of estimated position error for the Baseline estimation of Dataset-1 with removal of poorly positioned satellites

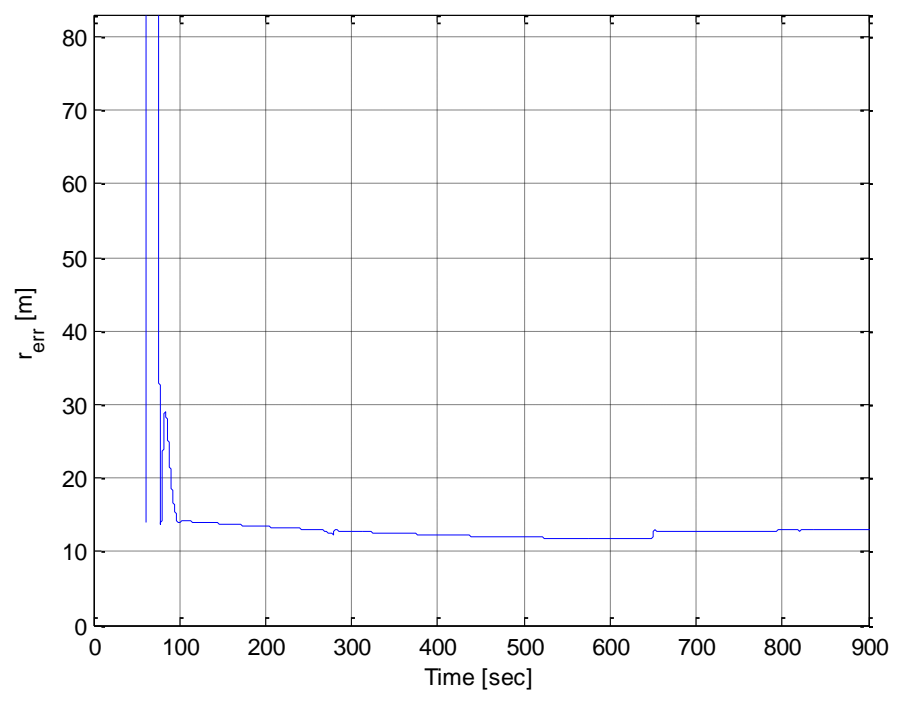

Figure 5.9: Radius of estimated position error for the CMTM estimation of Dataset-1 with removal of poorly positioned satellites

The evaluation conducted with Dataset-2 was included in this thesis because it has the potential for an increased estimation performance. This was assumed since the constant number 
of satellites and relatively constant standard deviation of the BESTXYZ position measurements. Figure 5.10 and Figure 5.11 on the next page are of the radius of estimated position error for each EKF measurement. Since all satellites are in view the entire time there should not have any large changes in the error. The minimums of the radius error when the $9^{\text {th }}$ satellite was no longer in view for the baseline and CMTM estimations for Dataset-1, were 16 and 11 meters respectively

Figure 5.10 and Figure 5.11 reveal an interesting result even though the number of viewed satellites was constant; the estimation error does not decrease over time. The error radius converges to a moderately high value. The difference of the baseline and CMTM estimations are clear, however even though the error converges the difference between the two is approximately ten meters. The removal of ten meters of error is a significant increase in performance with only the addition of precise satellite position estimates.

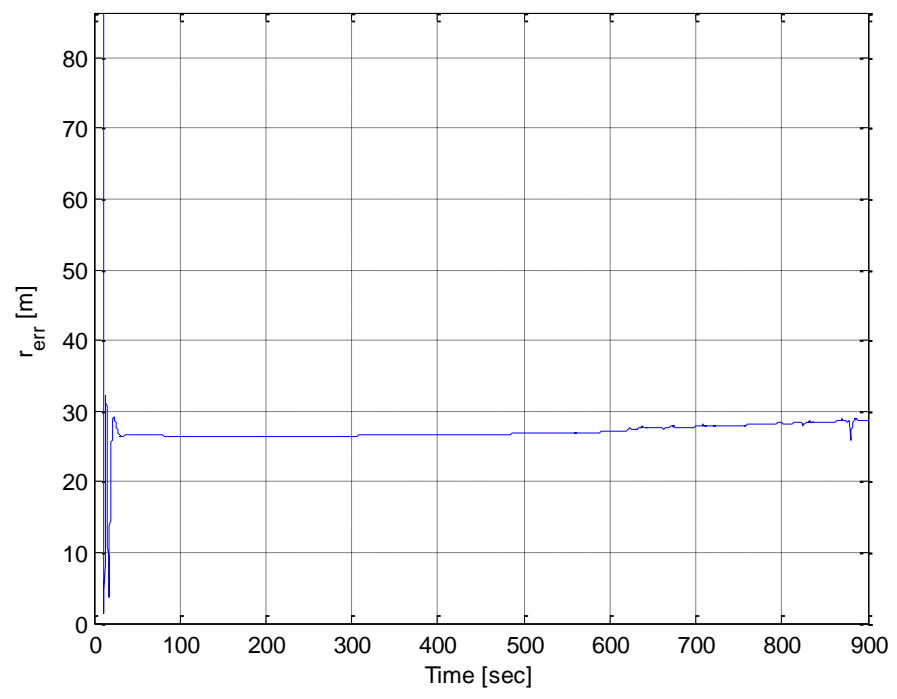

Figure 5.10: Radius of estimated position error for the Baseline estimation of Dataset-2 


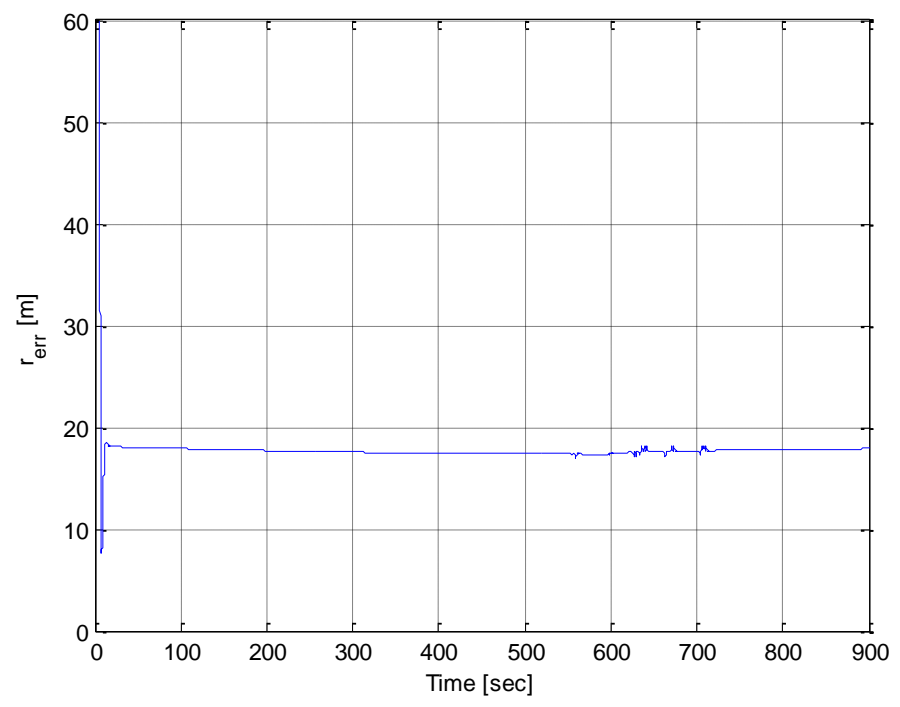

Figure 5.11: Radius of estimated position error for the CMTM estimation of Dataset-2

Even though the estimation converges to a moderately high value suspected reasons are: constellation geometry, and un-modeled errors. Once again by looking at the constellation geometry for Dataset-2 there were a couple of satellites that were not ideally positioned. The identified satellites in the figure below were assumed to be poorly positioned.
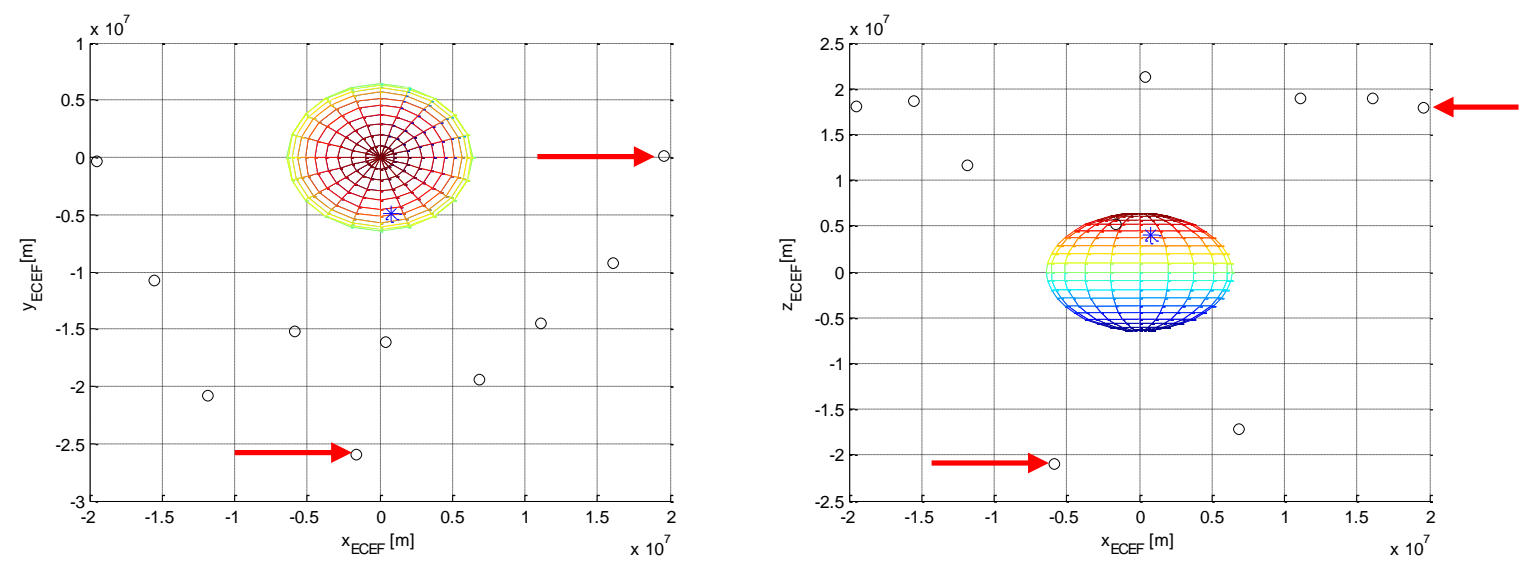

Figure 5.12: Orthogonal views of Dataset-2 constellation geometry 
The removal of these poorly positioned satellites once again improved the estimation performance for both cases, seen in Figure 5.13 and Figure 5.14. The baseline decreased by approximately 10 meters similarly as the change in Dataset-1. For this dataset there were only two satellites removed, this however interestingly changed the convergence time of the baseline from 30 to 40 seconds and barely changed the CMTM convergence time.

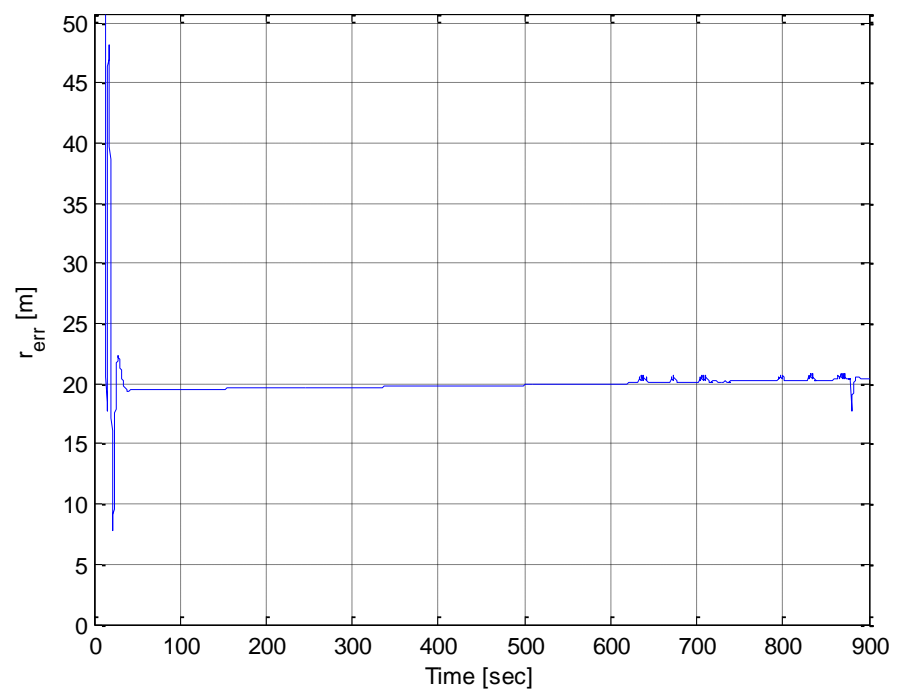

Figure 5.13: Radius of estimated position error for the Baseline estimation of Dataset-2 with poorly positioned satellites removed

The overall accuracy is quite high for all cases seen previously; however the precision does seemed biased. A possible reason for this could be due to datasets only being 15 minutes long which may not provide enough time for the normal distribution on all components assumption to be fully applicable. For Dataset-2, the BESTXYZ log seems to have converged to a small area with a smaller radius error. Figure 5.14 has the lowest radius error, and continually decreases with time. 


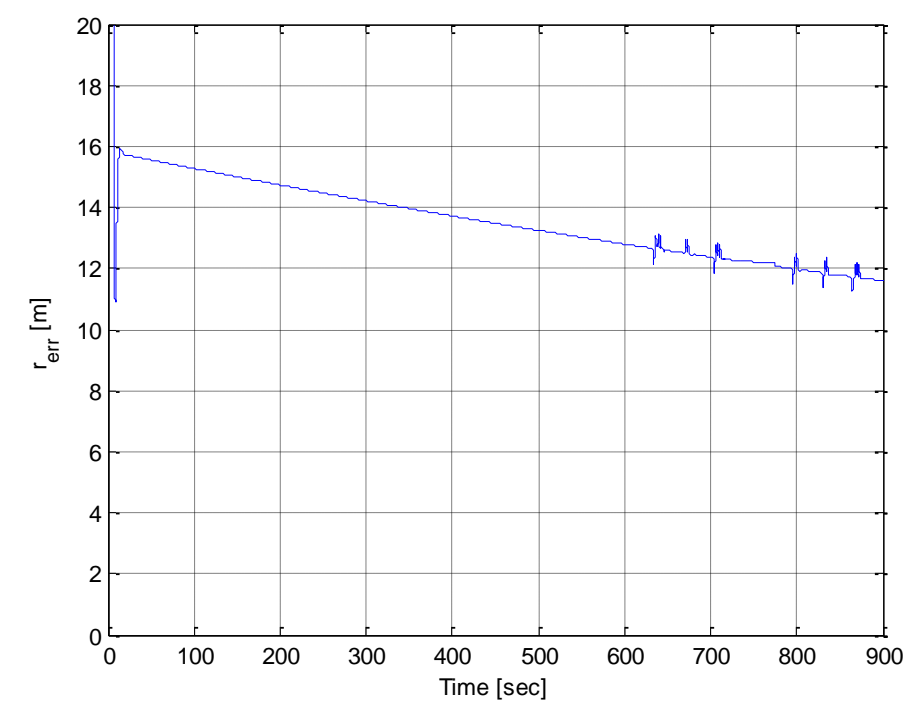

\section{Figure 5.14: Radius of estimated position error for the CMTM estimation of Dataset-2 with poorly positioned satellites removed}

The difference between the baseline and CMTM result once again is within a reasonable margin. The use of the CMTM decreased the radius error by 4-8 meters compared to the baseline. With the steady decrease in radius error a hopeful conclusion would be that with more time this would converge to zero. This will not most likely occur because the use of the atmospheric corrections within both sets of measurements, and un-modeled clock biases/drift for each satellite. Including IGS clock information for the viewed satellites would most likely decrease the position estimation drift caused by individual satellites.

Even though there is an improvement between using the baseline estimation and the CMTM estimation this is only a small portion of the information that could be used in determining the precise location of a GPS receiver in real-time. When using pseudorange measurements without a fault tolerance evaluation the use of the created measurements from the MATLAB tools will most likely perform better than an estimation algorithm that uses raw data or broadcast ephemerides. 


\section{Chapter 6 : Conclusions and Future Directions}

\subsection{Summary and Conclusion}

A significant portion of this graduate project was the development and advancement of the PAC aircraft avionic hardware. The use of these advancements allowed the collection of more information to be used in real-time. This provides a large amount of growth to be possible within the PAC aircraft research areas. From the development of the top PCB within the Gen-V system to the nose PCB, these hardware improvements were significant and useful to all research topics within the FCSL.

The development of the MATLAB tools provides an easy and accessible use of IGS products and raw data types. The variety of different data types that can be used within the MATLAB tools is extensive. These data types include raw broadcast ephemeris, precise product satellite positions and clock estimates, and ionospheric TEC maps of the globe. Within these data types other information is available such as Earth rotation parameters and polar motion. This allows for an introduction to IGS products and data to fellow researchers. The format in which these tools were created allows for expansions to easily be implemented.

An evaluation of the use of real-time information created from the MATLAB tools was conducted. This was completed by a static GPS receiver that recorded position estimates, satellite position estimates and time information. This was used in the creation of a baseline set of measurements. This information was refined such that erroneous measurements were removed. The creation of the MATLAB tools measurement used predicted product data from IGS to estimate the GPS satellites for a real-time application. These measurements were compared through the use of a four state extended Kalman filter. The states of the EKF were the GPS receiver's position in the ECEF frame and receiver clock bias.

The baseline EKF that used recorded satellite position provided a reasonable position estimation solution. The position estimation that used the created MATLAB tools measurement had a reduction of estimation error of approximately 10 meters when using all viewed satellites. The removal of poorly positioned satellites decreased both estimation errors however the CMTM results showed approximately a five meter improvement over the baseline. This created 
information in a real-time application decreased the position error by approximately one fourth less than the baseline estimation.

\subsection{Future Directions}

Implications can easily be hypothesized with the further integration of precise predicted products or models developed by past precise products a significant increase in accuracy and precision is possible. IGS information is just one component of information that can be used to improve a GPS position estimate. The incorporation of other products available has the potential to provide a cm-level accuracy of position estimation. Specifically the incorporation of clock information for each satellite would be useful for a real-time application.

The inclusion of INS information will also greatly increase the accuracy of a position estimation algorithm. For example, the inclusion of an in-depth stochastic model of inertial sensors would provide a more complete modeling of INS information. Another example would be if a GPS/INS formulation would also include magnetometer measurements. The fusion of more information sources should ultimately increase estimation performance.

The development of the MATLAB tools created here will be further enhanced at the FCSL. Expansions to incorporate other IGS product data are currently being conducted. Also the use of locally positioned Continuously Operation Reference Stations (CORS), stations apart of the NGS network, is also being pursued. The use of this high rate information could provide estimations of atmospheric properties, and relative positioning from the base station(s).

The integration of INS information has previously been conducted in a fault tolerant application in Gross's dissertation [2]. The information filter developed in this dissertation could be augmented to be a fault tolerant tightly-coupled attitude estimation algorithm employing the use of the IGU produced by the developed MATLAB tools. Within the FCSL, the incorporation of additional sources is currently being pursued by Dr. Yu Gu. 


\section{References}

[1] J. Kouba, "A guide to using International GPS Service (IGS) Products," Geodetic Survey Division Natural Resources Canada, Ottawa, Ontario, 2003.

[2] J. Gross, "Sensor Fusion Based Fault-Tolerant Attitude Estimation Solutions for Small Unmanned Aerial Vehicles," West Virginia University, Morgantown, WV, PhD Thesis 2011.

[3] Y. Gu et al., "Autonomous Formation Flight: Hardware Development," in Mediterranean Control Conference, Ancona, Italy, June, 2006.

[4] Y. Gu, B. Seanor, S. Gururajan, and M. Napolitano, "Integrated Avionics Systems for UAVs," in AIAA Guidance, Navigation and Controls Conference and Exhibit, Honolulu, HI, Aug. 2008.

[5] B. Seanor et al., "3 Aircraft Formation Flight Experiment," in Meterranean Control Conference, Ancona, Italy, June, 2006.

[6] MathWorks. (2011, December) MathWorks Academia. [Online]. http://www.mathworks.com/academia/faculty_center/includes/9364v04_MathWorks-Academia.pdf

[7] K. Borre, "The GPS Easy Suite-Matlab code for the GPS newcomer," GPS Solutions, vol. 7, no. 1, pp. 47-51, May 2003.

[8] T. Herring, "MATLAB Tools for viewing GPS velocities and time series," GPS Solutions, vol. 7, no. 3, pp. 194-199, 2003.

[9] M. Scharber, Y. Bock, and B Gilmore, "Software Tools for Accessing the GPS Seamless Archive," GPS Solutions, vol. 7, no. 4, 2004.

[10] X. Chang and T. Zhou, "MILES: MATLAB package for solving Mixed Integer LEast Squares problems," GPS Solutions, vol. 11, no. 4, pp. 289-294, November 2007.

[11] Z. Dai, S. Knedlik, and O. Loffeld, "A MATLAB toolbox for attitude determination with GPS multiantenna systems," GPS Solutions, vol. 13, no. 3, pp. 241-248, July 2009.

[12] M. Horemuz and J. V Andersson, "Polynomial interpolation of GPS satellite coordinates," GPS Solutions, vol. 10, pp. 67-72, January 2006.

[13] M. Schenewerk, "A brief review of basic GPS orbit interpolation ," GPS Solutions, vol. 6, no. 4, pp. 265-267, 2003.

[14] Y. Feng and Y Zheng, "Efficient interpolations to GPS orbits for Wide Area Application," GPS Solutions, vol. 9, pp. 273-282, May 2005.

[15] D. E Gaylor, "Integrated GPS/INS navigation system design for autonomous spacecraft rendezvous," The University of Texas, Austin, PhD Thesis 2011.

[16] Navsys Corp. (2011, September) GPS Signal Simulation Toolbox. [Online]. http:/www.navs ys.com/products/toolbox.htm

[17] Tae-Yun et al., "Development of the GPS Simulator for Driving Simulators," in International Joint 
Conference, 2006, pp. 2213-2137.

[18] Julien et al,, "A Complete Software-Based IF GNSS Signal Generator for Software Receiver Development," in Proceedsing of the 17th International Technical Meeting of the Satellite Division of The Insitute of Navigation, Long beach, CA., 2004, pp. 2146-2157.

[19] W. Liduan, Chuanrun Z., Wenrui J., and Z. Xingqun, "Enhanced GPS Measurements Simulation for Space-oriented Navigation System Design," Chinese Journal of Aeronautics, vol. 23, no. 4, pp. 438446, August 2010.

[20] Spirent. (2011, September) What is GPS Simulation? [Online]. http://www.spirent.com/Positioningand-Navigation/What_is_GPS_Simulation.asp $\mathrm{x}$

[21] P Kashyap, "Real Time Software GPS Receiver Implementation," The University of Texas, San Antonio, MS Thesis 2009.

[22] J Um, "Relative Navigation and Attitude Determination Using a GPS/INS Integrated System Near the International Space Station," The University of Texas, Austin, PhD Thesis 2001.

[23] T. Ebinuma, "Precision Spacecraft Rendezvous Using Global Positioning System: An Integrated Hardware Approach," The University of Texas, Austin, PhD Thesis 2001.

[24] A. Brown and N. Gerein, "Advanced GPS Hybrid Simulator Architecture," in Proc. of ION 57th Annual Meeting , Alburquerque, NM, June, 2001.

[25] P. Gustavsson, "Development of a MatLab-based GPS Constellation Simulation for Navigation Algorithm Developemnts," Lulea University of Technology, Kiruna, MS Thesis 2005.

[26] C. Abart, "Simulating GNSS constellations - GPS, Galileo and SBAS," in ELMAR, 2008. 50th International Symposium, Zadar, Sept. 2008 , pp. 569 - 572.

[27] P Boulton et al., " Proposed Models and Methodologies for Verification Testing of AGPS-Equipped Cellular Mobile Phones in the Laboratory," in Proceedings of the 15th International Technical Meeting of the Satellite Division of The Institute of Navigation (ION GPS 2002), Portland, OR., September 2002, pp. 200 - 212.

[28] K.A. Redmill, "Sensor and data fusion design and evaluation with a virtual environment simulator," in Proceedings of the IEEE, 2000, pp. 668-674.

[29] O. Julien, B. Zheng, L. Dong, and G. Lachapelle, "A Complete Software-Based IF GNSS Signal Generator for Software Receiver Development," in ION GNSS Sept. 21-24, Long Beach, CA, 2004.

[30] J. Rankin, "An error model for sensor simulation GPS and differential GPS," in Position Lovatoin and Navigation Symposium, IEEE, Las Vegas, NV, USA , 1994, pp. 260 - 266.

[31] L. Dong, "IF GPS Signal Simulator Development and Verification," University of Calgary, MS Thesis 2003.

[32] X. Kong, "GPS Modeling in Frequency Domain," in The 2nd International on Wireless Broadband and Ultra Wideband Communications, August, 2007, p. 61.

[33] B.L. Malleswari, I.V. MuraliKrishna, K. Lalkishore, M. Seetha, and P.H. Nagaratna, "The Role of Kalman Filtering in the Modelling of GPS Errors," Journal of Theoretical and Applied Information 
Technology, January 2008.

[34] X Yuana, J. Fua, H. Suna, and C. Toth, "The application of GPS precise point positioning technology in aerial triangulation," ISPRS Journal of Photogrammetry and Remote Sensing, vol. 64, no. 6, pp. 541-550, November 2009.

[35] M. Honda, M. Murata, and Y Mizukura, "GPS Precise Point Positioning Methods Using IGS Products for Vehicular Navigation Applications," in SICE-ICASE International Joint Conference, Bexco, Busan, Korea, 2006.

[36] O. Bogdanov and A. Cepe, "Precise orbit determination for GPS and GLONASS satellites on the basis of IGS data," in 5th International Conference on Recent Advances in Space Technologies (RAST), 2011, pp. 915 - 919.

[37] D. Hobbs and Bohn. Precise Orbit Determination for Low Earth Orbit Satellites. [Online]. http://www.astro.lu.se/ david/papers/Hobbs_POD_annalsIV_Final.pdf

[38] A. Komjathy, "Global Ionospheric Total Electron Content Mapping Using the Global Positioning System," University of New Brunswick, Frederiction, N.B. Canada, PhD Thesis 1997.

[39] M. Grewal, L. Weill, and A. Andrews, Global Positioning Systems, Inertial Navigation, and Integration, 2nd ed. Hoboken, New Jersey, United States of America: John WIley \& Sons, Inc., 2007.

[40] The Aerospace Corporation. (2011, September) Education; GPS Primer; GPS Timeline. [Online]. http:/www.aero.org/education/primers/gps/gpstimeline.html

[41] D. Jefferson and Y Bar-Sever, "Accuracy and Consistency of Broadcast GPS Ephemeris Data," in Proceedings of the 13th International Technical Meeting of the Satellite Division of The Institute of Navigation, 2000, pp. 391-395.

[42] Pratap Misra and Per Enge, Global Positioning System, Signals, Measurements, and Performance, 2nd ed. Lincoln, Mass., United States of America: Ganga-Jamuna Press, 2011.

[43] E. Kaplan and C. Heagarty, Understanding GPS Principles and Applications, 2nd ed. Norwood, MA: Arttech House, 2006.

[44] R. Bate, D. Mueller, and J. E. White, Fundamentals of astrodynamics. New York, USA: Dover Publications, 1971.

[45] Y. M. Wang, J. Saleh, X. Li, and D.R. Roman, "The US Gravimetric Geoid of 2009 (USGG2009): model development and evaluation," Journal of Geodesy, no. 0949-7714, pp. 1-16, September 2011.

[46] International Earth Rotation and Reference Systems Service. (2010, February 8) Home; Background; Earth Rotation; Coordinated Universal Time (UTC). [Online]. http:/www.iers.org/nn_10398/IERS/EN/Science/EarthRotation/UTC.html

[47] R. Langley, "Time, Clocks, and GPS," GPS World Magazine, pp. 38-42, November/December 1991.

[48] J. Tsui, Fundamentals of Global Positioning System Receivers: A Software Approach. New Jersey, USA: John Wiley \& Songs, 2005.

[49] NAVSTAR GPS. (1995, June) Global Positiong System Standard Positioning Service Signal 
Specification. [Online]. www.pnt.gov/public/docs/1995/signalspec1995.pdf

[50] J. Farrell, Aided navigation : GPS with high rate sensors. New York, USA: McGraw-Hill, 2008.

[51] B. W. Remondi, "Computing satellite velocity using the broadcast ephemeris," GPS Solutions, vol. 8, no. 3, pp. 181-183, September 2004.

[52] W. Zhang, Me. Ghogho, and L. E. Aguado, "Extension of GPS Broadcast Ephemeris to Deter-mine Satellite Velocity and Acceleration," in ENC-GNSS, Naples, Italy, 2009.

[53] S. Ackerman and J. A. Knox, Meteorology: Understanding the Atmosphere, 2nd ed.: Brooks Cole, 2006.

[54] J. F. Kasting and T.P. Ackerman, "Climatic Consequences of Very High Carbon Dioxide Levels in the Earth's Early Atmosphere," in Science., Dec. 12, 1986, pp. 1383-1385.

[55] A, Hauschild and O. Montenbruck, "Kalman-filter-based GPS clock estimation for near real-time positioning," GPS Solutions, vol. 13, no. 3, pp. 173-182, July 2009.

[56] Y.S. Shmaliy, A.V. Marienko, and A.V. Savchuck, "GPS-Based Kalman Estimation of Time Error, Frequency Offset and Aging," in 31st Annual Precise Time and Time Interval (PTTI) Meeting , 1999.

[57] L. Arceo-Miquel, Y.S. Shmaliy, and O. Ibarra-Manzano, '"'Optimal Synchronization of Local Clocks by GPS 1PPS Signals Using Predictive FIR Filters," in , IEEE Transactions on Instrumentation and Measurement, June 2009, pp. 1833-1840.

[58] IGS Central Bureau. (2011, October) International GNSS Service - Home page. [Online]. http://igscb.jpl.nasa.gov/

[59] International GNSS Central Bureau, "IGS," International GNSS Central Bureau, Pasadena, CA, PDF Information Sheet.

[60] International GNSS Service. (2009, July) IGS Products. [Online]. http://igscb.jpl.nasa.gov/components/prods.html

[61] J. M. Dow, R. E. Neilan, and C. Rizos, "The International GNSS Service in a changing landscape of Global Navigation Satellite Systems," Journal of Geodesy, vol. 83, no. 7, p. 689, 2009-07-01.

[62] IGS. (2010) IGS 2010 Workshop Summary Recommendations. [Online]. ftp//igscb.jpl.nasa.gov/pub/resource/pubs/IGS_2010_Newcastle_Workshop_Recommendataions.pdf

[63] M. J. Merrigan, E. R. Swift, R. F. Wong, and J. T. Saffel, "A Refinement to the World Geodetic System 1984 Reference Frame," in Proceedings of the 15th International Technical Meeting of the Satellite Division of The Institute of Navigation (ION GPS 2002), Portland, OR., September 2002, pp. 1519-1529.

[64] National Geodetic Survey (Ray, J.). (2011, November) Figures and Statistics from the IGS UltraRapid GPS Orbit Combination. [Online]. http://acc.igs.org/igsacc_ultra.html

[65] R. E. Kalman and R. S. Bucy, "New Results in Linear Filtering and Prediction Theory," Journal of Basic Engineering (Transactions of ASME), pp. 95-108.

[66] D. Simon, Optimal State Estimation. Hoboken, New Jersey: John Wiley \& Sons, 2006. 
[67] B. Quine, , J. Uhlmann, and Hugh D.W., "Implicit Jacobians for Linearised State Estimation in Nonlinear Systems," in American Control Conference, IEEE Press, June - 1995.

[68] J. Gross, Y. Gu, B. Seanor, S. Gururajan, and M. Napolitano, "Advanced Research Intergrated Avionics (ARIA) System for Fault-Tolerant Flight Research," in AIAA Guidance, Navigation and Controls Conference and Exhibit, Chicago, USA, 2009.

[69] NovAtel, "OEMV® Family Firmware Reference Manual," Reference Manual OM-20000094 Rev 8, 2010/05/14.

[70] Netburner. (2011, Nov.) Netburner, Home, Products, Core Modules, MOD5213. [Online]. http://www.netburner.com/products/core_modules/mod5213.html

[71] P3 America. (2004) Single-Turn Potentiomter Series - MP 1545A.

[72] Sensor Technics. HCE Series - Miniature amplified pressure sensors. E/ 11652/ E.

[73] Measurement Specialties. (2008, Month) HTM2500 - Temperature and Relative Humidity Module.

[74] Opti-Logic. (2007, October Rev. 2.8) Operating Instructions for the RS232 Laser Rangfinder Module.

[75] AirNav.com. (2011, November) AirNav.com-WV23-Louis Bennet Field Airport. [Online]. http://www.airnav.com/airport/WV23

[76] B. W. Remondi, "Distribution of Global Positioning System Ephemerides by the National Geodetic Survey," in Presented at the First Conference on Civil Applicaions of GPS - Institute of Navigation, September 12,1985, p. 8.

[77] B. W. Remondi, "Extending the National Geodetic Survey Geodetic Orbit Formats," NOAA, NOAA Technical Report 133 NGS 46 85, 1989.

[78] B. W. Remondi, "NGS Second Generation ASCII and Binary Orbit Formats and Associated Interpolated Studies," in Proceedings of the Twentieth General Assembly, International Union of Geodesy and Geophysics, Vienna, Austria, August 11-24, 1991, p. 28.

[79] S. Hilla. (2007, November) The Extended Standard Product 3 Orbit Format (SP3-c). [Online]. http://www.ngs.noaa.gov/orbits/sp3c.txt

[80] P. R. Spofford and B. W. Remondi. The National Geodetic Survey Standard GPS Format SP3. [Online]. http://igscb.jpl.nasa.gov/igscb/data/format/sp3_docu.txt

[81] J. Griffiths and J. R. Ray, "On the precision and accuracy of IGS orbits," Journal of Geodesy, vol. 83, no. 3, p. 277, March 2009.

[82] W. Gurtner, "Rinex: The Receiver Independent Exchange Version 2," Astronomical Institute, University of Berne, Rinex 2998.

[83] "ICD-GPS-200," Navstar GPS Space Segment/Navigation User Interface, 1993.

[84] F. Barchesky, J. Gross, Y. Gu, M. Rhudy, and M.R. Napolitano, "Development of a GPS/INS Sensor Fusion Simulation Environment Using Flight Data," in 2011 AIAA Modeling and Simulation Technologies Conference, Portland, OR, 2011.

[85] D. A. Vallado and W.D. McClain, Fundamentals of Astrodynamics and Applications, 3rd ed. New 
York, NY, USA: McGraw-Hill, 2007.

[86] C-T. Weng, Y-C. Chien, C-L. Fu, W-G. Yau, and Y.J. Tsai, "A Broadcast Ephemeris Extension Method for Standalone Mobile Apparatus," in Proceedings of the 22nd International Technical Meeting of The Satellite Division of the Institute of Navigation, Savannah, GA, September 2009, pp. 2108-2114.

[87] S. Malys and M. J. Ortiz, "Geodetic absolute positioning with differenced GPS carrier beat phase data," in Proceedings of the 5th International Symposium on Satellite Positioning, Las Cruces, 1989.

[88] B. W. Remondi, "NGS second generation ASCII and binary orbit formats and associated interpolation studies," in 20th General Assembly of the IUGG, Vienna, 1991, pp. 11-24.

[89] J. Ruibo and L. Xiaoqiang, "Using Fourier series to fit The GPS precise ephemeris," in 2010 International Conference on Computer Application and System Modeling (ICCASM), 22-24 Oct. 2010, pp. V11-96-V11-99.

[90] G. Petit and B. Luzum, "IERS Conventions (2010)," Internation Earth Rotation and Reference Systems Service (IERS), Frankfurt am Main 2010), IERS Technical Note No. 362010.

[91] Guochang Xu, Orbits. Berlin: Springer, 2008.

[92] Koblick. (2010, July) Convert ECEF to ECI Coordinates. Matlab Function.

[93] P. S. Maybeck, "Stochastic models, estimation and control," Mathematics in Science and Engineering, vol. 141, no. 2.

[94] NovAtel. (2011) OEMV-1 $\quad$ Product $\quad$ Sheet. http:/www.no vatel.com/assets/Documents/Papers/OEMV-1_Series.pdf 


\section{Appendix A.}

A.1 Relevant Constants Used

\begin{tabular}{|c|c|c|}
\hline Term & Value & Units \\
\hline Speed of light $^{1}$ & $c=299729.458$ & $\mathrm{~km} / \mathrm{sec}$ \\
\hline Geocentric gravity constant $^{1}$ & $\mu=3986400.440 \pm 0.002$ & $\mathrm{~km}^{3} / \mathrm{sec}^{2}$ \\
\hline WGS84 geocentric gravity constant $^{2}$ & $\mu=3986400.5$ & $\mathrm{~km}^{3} / \mathrm{sec}^{2}$ \\
\hline Earth rotation rate $^{2}$ & $d \Omega_{E} / d t=7.2921151467 \times 10^{-5}$ & $\mathrm{rad} / \mathrm{s}$ \\
\hline Solar to Sideral Day Ratio $^{3}$ & $d \theta / d t=1.00273781191135448$ & $\mathrm{rev} / \mathrm{day}_{U T 1}$ \\
\hline
\end{tabular}

For more information on the above values see the following references:

${ }^{1}$ J. Farrell $[\mathbf{4 8}]$.

${ }^{2}$ Taken from B. W. Remondi [36].

${ }^{3}$ Pg. 18 of IERS Convections (2010) [79]. 


\section{Appendix B.}

\section{B.1 Tables from RINEX-2.10 Format Document [82]}

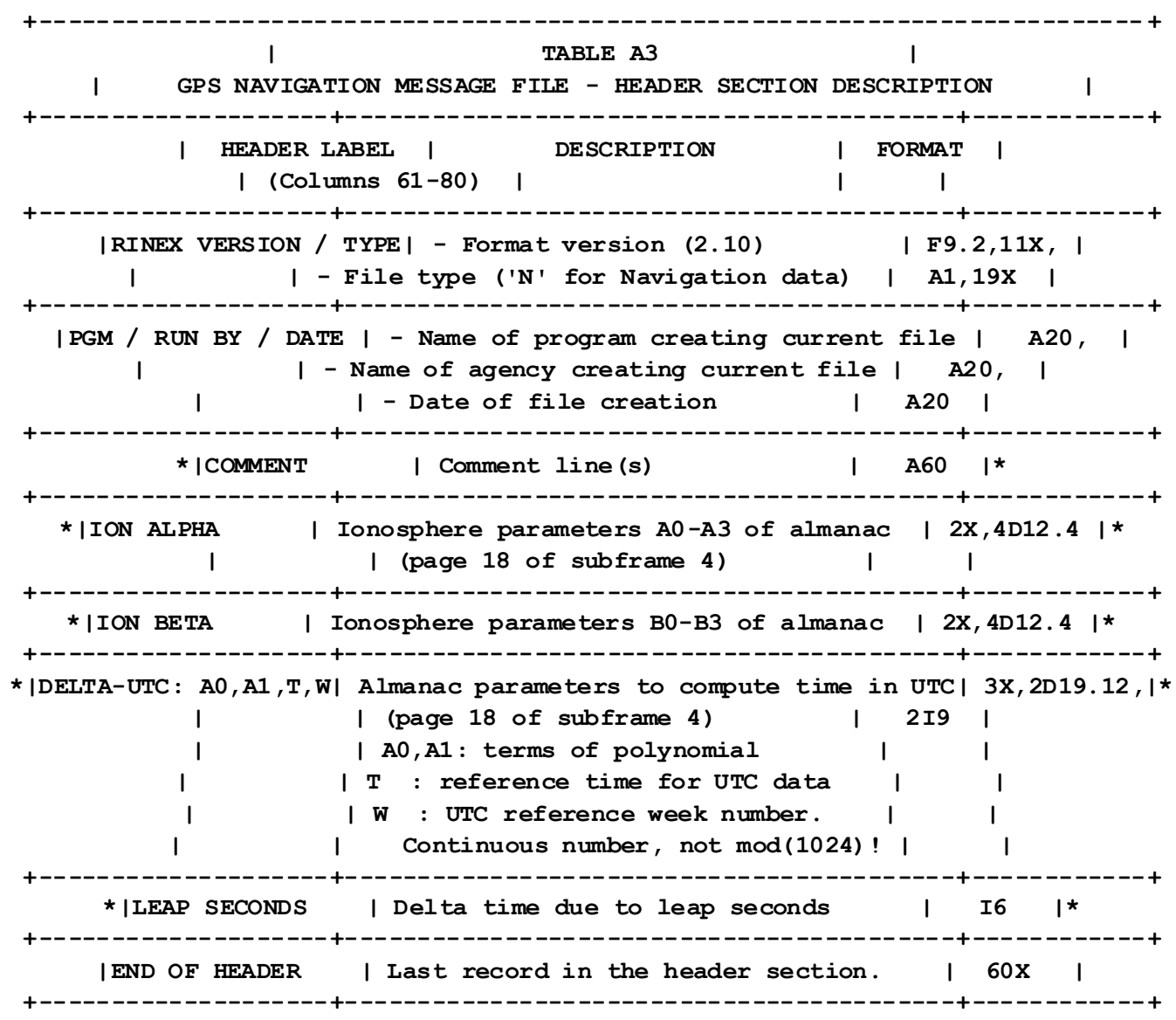

Records marked with * are optional 


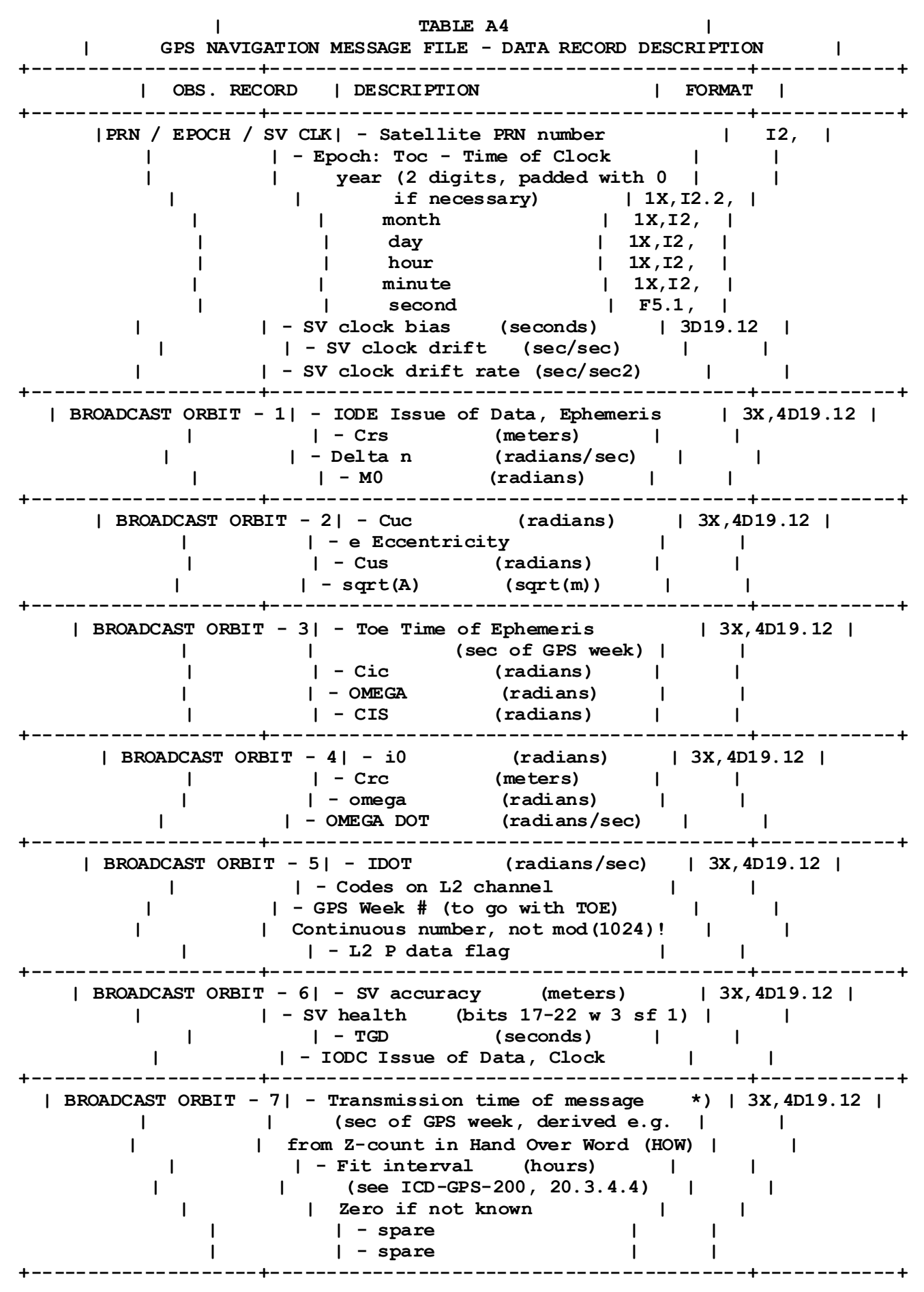

*) Adjust the Transmission time of message by -604800 to refer to the reported week, if necessary 